Chapman University

Chapman University Digital Commons

Winter 1-27-2020

\title{
Experienced Pediatric Oncology Nurses Using Self-Reflective Practices in the Clinical Setting: A Descriptive Phenomenological Investigation
}

Daniel Josue Bonilla

danbonilla123@gmail.com

Follow this and additional works at: https://digitalcommons.chapman.edu/education_dissertations

Part of the Educational Leadership Commons, and the Leadership Studies Commons

\section{Recommended Citation}

Bonilla, D. (2020). Experienced pediatric oncology nurses using self-reflective practices in the clinical setting: A phenomenological investigation [Doctoral dissertation, Chapman University]. Chapman University Digital Commons. https://doi.org/10.36837/chapman.000119

This Dissertation is brought to you for free and open access by the Dissertations and Theses at Chapman University Digital Commons. It has been accepted for inclusion in Education (PhD) Dissertations by an authorized administrator of Chapman University Digital Commons. For more information, please contact laughtin@chapman.edu. 
Experienced Pediatric Oncology Nurses Using Self-Reflective Practices in the Clinical Setting:

A Descriptive Phenomenological Investigation

A Dissertation by

Daniel Josue Bonilla

Chapman University

Orange, California

College of Educational Studies

Submitted in partial fulfillment of the requirements for the degree of

Doctor of Philosophy in Education

January 2020

Committee in charge:

Whitney McIntyre Miller, Ph.D., Chair

Penny S. Bryan, Ph.D.

Michael Andersen, P.T., D.P.T.

Dawn Hunter, Ph.D. 
The dissertation of Daniel Josue Bonilla is approved.

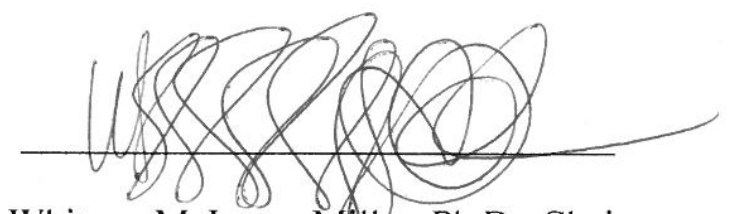

Whitney McIntyre Miller, Ph.D., Chair

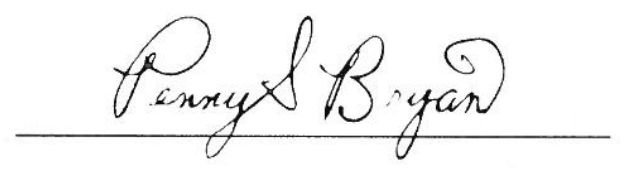

Penny S. Bryan, Ph.D.

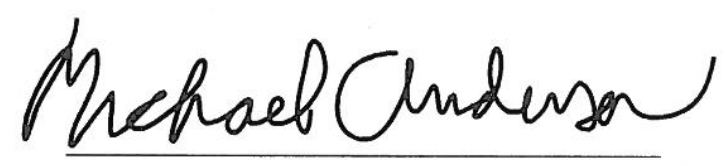

Michael Andersen, P.T., D.P.T.

\section{Dawn Aunter}

Dawn Hunter, Ph.D.

January 2020 
Experienced Pediatric Oncology Nurses Using Self-Reflective Practices in the Clinical Setting:

A Descriptive Phenomenological Investigation

Copyright $@$ January 2020

by Daniel Josue Bonilla 


\section{ACKNOWLEDGMENTS}

Completion of this dissertation could not have been possible without the amazing leadership, daily support, and passionate guidance from the faculty and staff in the Attallah College of Educational Studies at Chapman University. I specifically acknowledge and thank all four of my committee members, beginning with my doctoral teacher and dissertation chair. Dr. Whitney McIntyre Miller was the first to hear my enthusiasm for developing this dissertation and she has continued to be there for me every step of the way. Dr. Penny S. Bryan was the first faculty member I met at Chapman University. Although she retired during the Spring 2018 semester after 24 years at Chapman, she continued her support and has helped me develop a dissertation of which I am proud. Dr. Dawn Hunter is an amazing educator and human being whom I admire. She was integral to my success as a doctoral student and future educator. Dr. Michael Andersen was invited to join my committee from the University of Southern California. I am thankful to Dr. Andersen for his commitment to my success both as a doctoral student and healthcare professional. I truly respect and admire the amount of time and energy each committee member has invested in my education. To my amazing familia, specifically Dad and Mom, los quiero mucho y soy un Doctor por el sacrificio y el amor que me han dado desde el principio. To big brother and big sister, words cannot express how thankful I am to have your

love and support throughout my pursuit of higher education. To Kerinda, my love, thank you for the daily encouragement and for always being there when I needed you. And finally, to all my family and friends, thank you for always sticking by my side and having my back no matter what. 


\begin{abstract}
Experienced Pediatric Oncology Nurses Using Self-Reflective Practices in the Clinical Setting:
\end{abstract}

A Descriptive Phenomenological Investigation

by Daniel Josue Bonilla

Pediatric oncology nurses can develop hazardous feelings of burnout over decades of clinical practice (Boyle \& Bush, 2018). Interventions that help decrease burnout and improve professional development are reflective practices (Caldwell \& Grobbel, 2013). Currently, there is a paucity of information on pediatric oncology nurses with 10 or more years of experience and how they use self-reflection to cope with workplace stressors. The purpose of this study was to explore how expert-level pediatric oncology nurses describe their experiences using selfreflective practices in the clinical setting. An interview-based exploration of the lived experiences of participants was necessary to understand the unique self-reflective practices currently used among nurses. Descriptive phenomenological methods were used, and data were organized and analyzed using the modified Stevick-Colaizzi-Keen method (Moustakas, 1994). Convenience and snowball sampling procedures were used. Six nurses fit the inclusion criteria and consented to participate in this study. Each participant completed three interviews. Results of the study were arranged in two categories: (a) the experience of using self-reflective practices in the clinical setting and (b) the experience of using self-reflective practices away from the clinical setting. In the clinical setting, experienced pediatric oncology nurses used self-reflection to develop better ways of interacting with patients, families, and colleagues. Using self-reflection to cope with stress and burnout occurred less in the clinical setting and more when experienced nurses reflected with other nurses, had moments of solitude, or when they were driving home from work. These results have implications for current nursing educators and nurses looking to 
develop skillsets to help reduce the harmful effects of stress and burnout in the clinical setting and maintain a productive career. 


\section{TABLE OF CONTENTS}

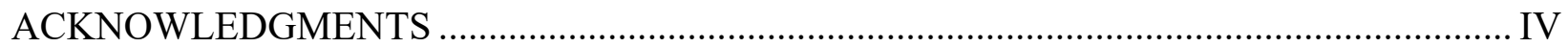

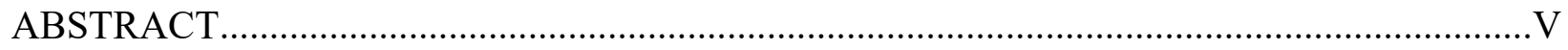

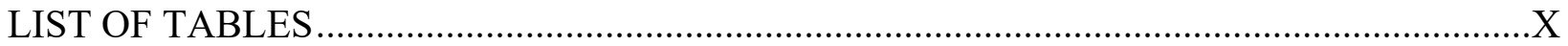

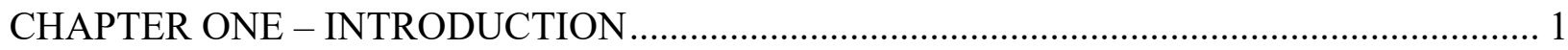

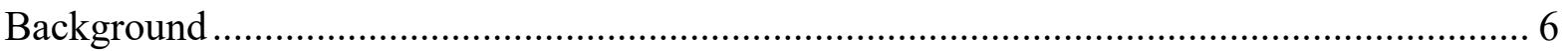

Reflection and the Role of Expert Nurses.................................................................... 7

The Art of Caring and Reflective Narratives ............................................................... 8

Reflection and Managing Emotions......................................................................... 9

Reflection and Managing Moral Distress ................................................................ 9

Student Nurses and Self-Reflective Diaries or Journals ................................................. 10

Preparing Nurses to Cope With Workplace Stressors .......................................................... 11

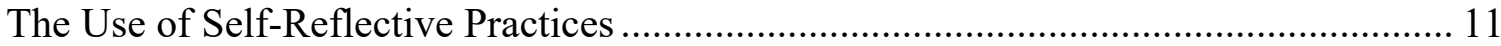

Reflection and Emotional Wellbeing..................................................................... 12

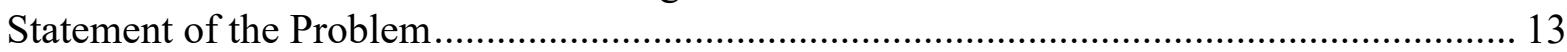

Nurses' Needs Come Second to the Patients' Needs...................................................... 13

No Time for Self-Care Leads to Stress and Burnout .................................................... 14

Nurses Need Time for Self-Reflection .................................................................. 15

Reflection and Experienced Pediatric Oncology Nurses ................................................... 16

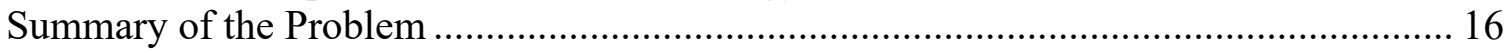

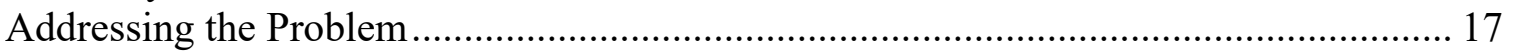

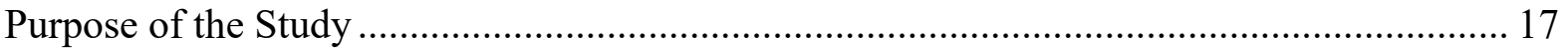

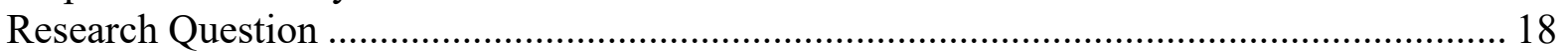

Methods Used to Address the Research Question ......................................................... 19

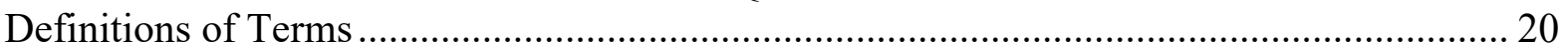

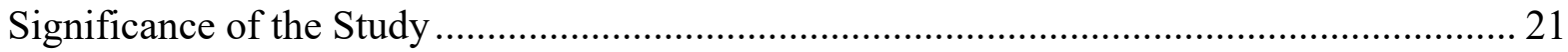

Subsequent Contribution......................................................................................... 22

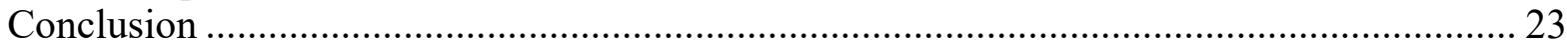

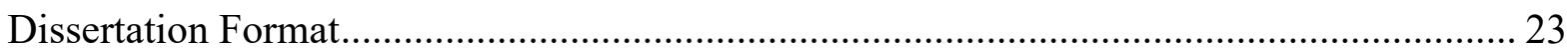

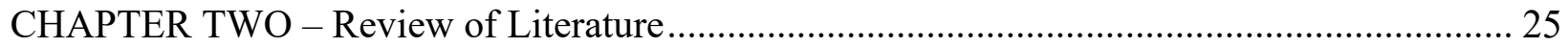

Theoretical Underpinnings of Reflective Practice in Nursing .............................................. 26

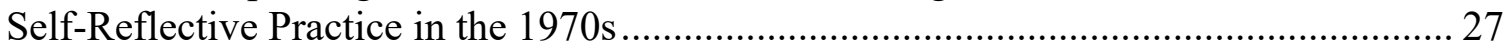

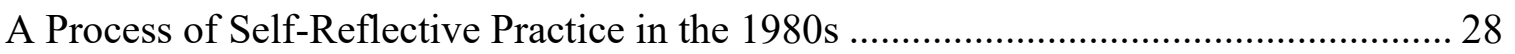

Reflective Practice in the 1990s..................................................................................... 32

A Summary of Self-Reflective Practices From the 1970s Through the 1990s................ 33

Emotional Intelligence, Self-Awareness, and Self-Reflection ........................................ 34

Nursing and the Skills Required for Using Self-Reflective Practices .............................. 36

Nurses and Time Spent Using Reflective Practice …………......................................... 37

Barriers Nurses Encounter When Using Reflective Practice............................................ 38

A Summary of Self-Reflective Practice for Nursing Professionals .................................. 39

A Guide for Nursing Students to Use Reflective Practice.................................................. 40 
Managing Emotions Triggered by Reflective Practice...................................................... 40

Care for Critical Patients and Reflective practices ………….......................................... 43

A Summary of Nursing Students and Their Perceptions Using Self-Reflection .............. 46

Nurses and the Ramifications of Using Self-Reflective Practices..................................... 47

Reflective Practice Intervention................................................................................ 48

Reflective Practice Groups ..................................................................................... 50

Summary and Key Findings of Reflective Practice in Nursing ....................................... 50

Review of the Literature on Pediatric Oncology Nursing ....................................................... 51

Methods Researchers Used to Explore Self-Reflective Practices.................................... 53

A Need to Continue Exploring Self-Reflective Practices.................................................. 59

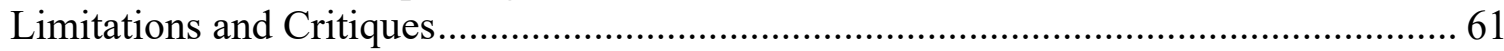

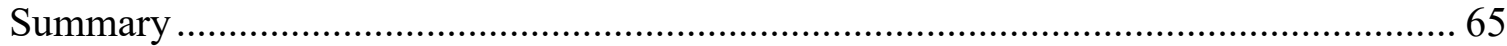

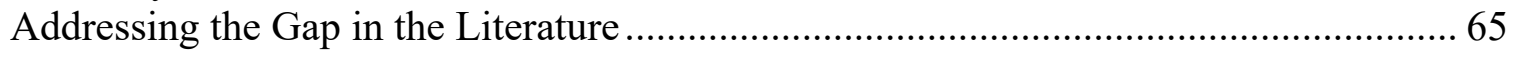

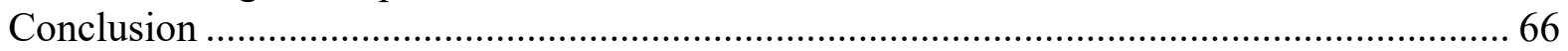

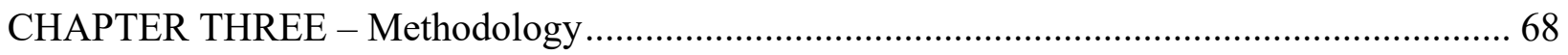

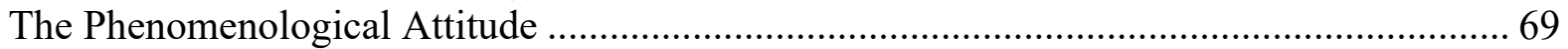

Embodiment of the Phenomenological Attitude …………............................................ 70

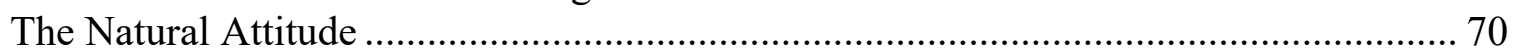

Researcher's Subjectivity Statement.......................................................................... 71

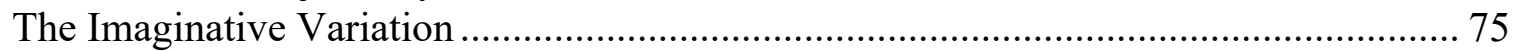

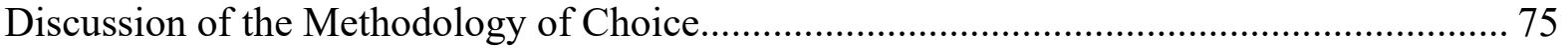

Husserl's (Descriptive) Phenomenology ...................................................................... 76

Hermeneutic (Interpretive) Phenomenology.................................................................. 77

Objects That Appear to the Mind................................................................................ 77

Methods for Using Transcendental Phenomenology ..................................................... 84

Rationale for Choosing Transcendental Phenomenology ...................................................... 88

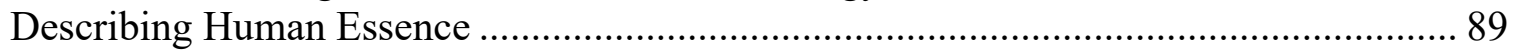

Deriving Knowledge From Lived Experience........................................................... 89

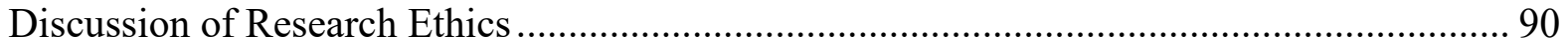

Developing Rapport and Trust............................................................................. 90

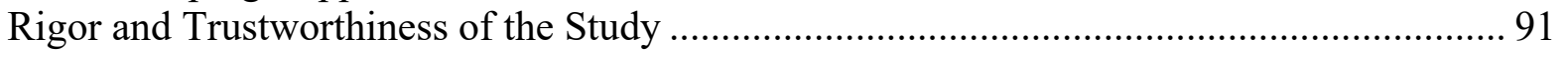

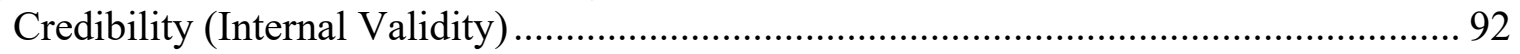

Member Checking ................................................................................................ 92

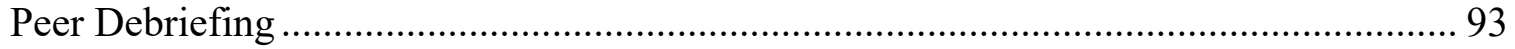

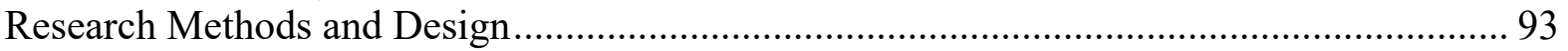

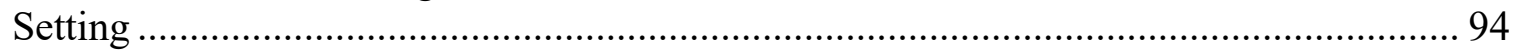

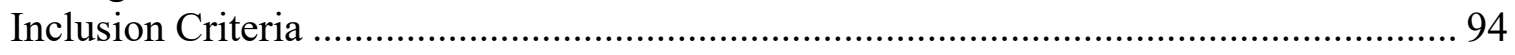

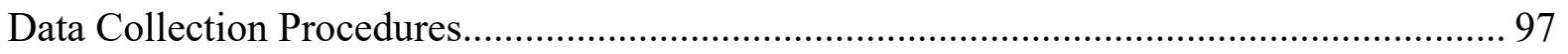

A Structure for a Phenomenological Interview …………............................................ 97

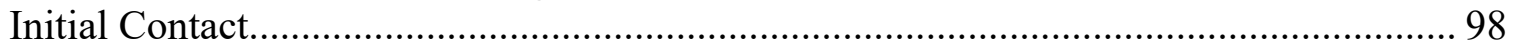

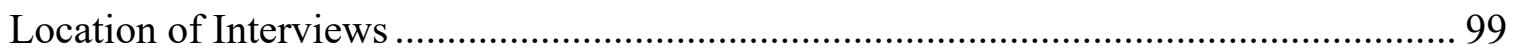

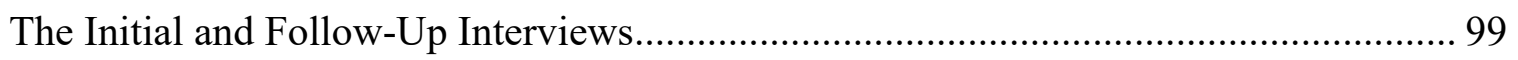

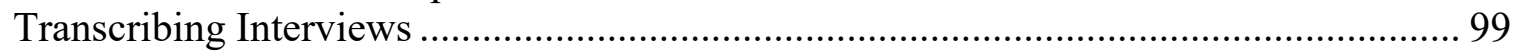

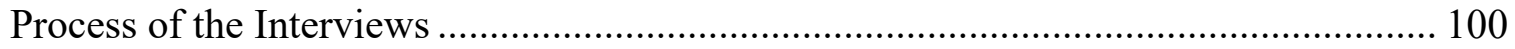

Bracketing Throughout Data Collection..................................................................... 101 
Data Analysis Method............................................................................................... 102

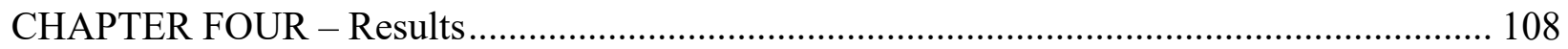

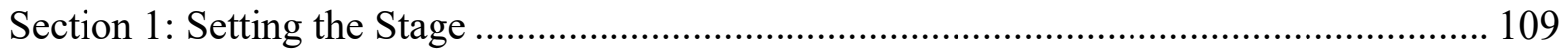

Defining Self-Reflective Practices............................................................................... 109

Advantages of Using Self-Reflection ………………................................................ 112

How Nurses Learned to Use Self-Reflective Practices............................................... 123

Making Time for Clinical-Based Reflective Practice …….......................................... 126

Section 2: The Experience of Using Self-Reflective Practice in the Clinical Setting .......... 127

Challenges to Using Clinical-Based Reflective Practice .................................................. 129

Section 3: The Experience of Using Self-Reflective Practice Away From the .................... 133

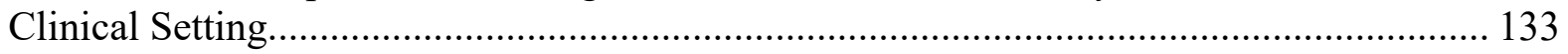

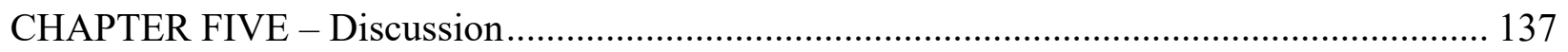

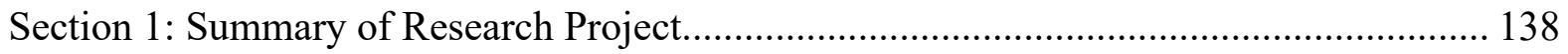

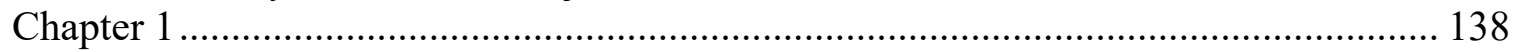

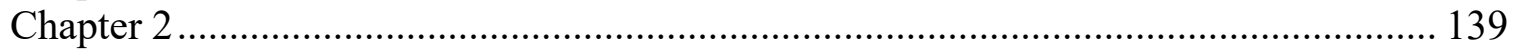

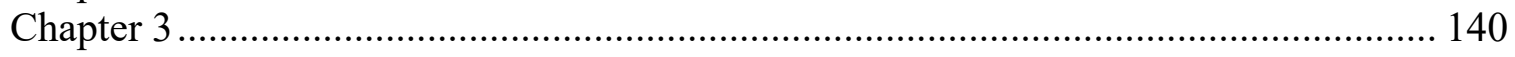

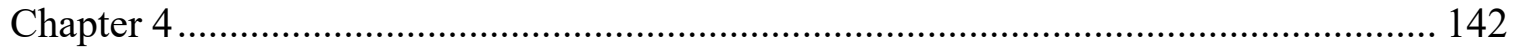

Section 2: Discussion of the Findings............................................................................. 143

Why Participants Used Self-Reflective Practices ........................................................ 144

How Participants Described Using Self-Reflective Practices ....................................... 145

Section 3: Implications and Significance........................................................................ 149

Implications for Nursing Education........................................................................ 150

Implications for Nursing Practice ……………………......................................... 153

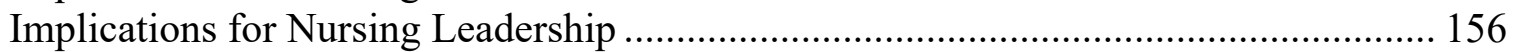

Significance and Transferability of the Study ............................................................ 158

Directions for Future Research .............................................................................. 159

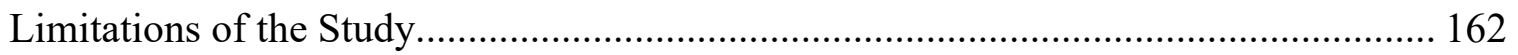

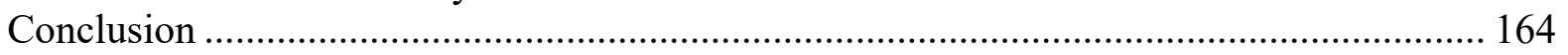

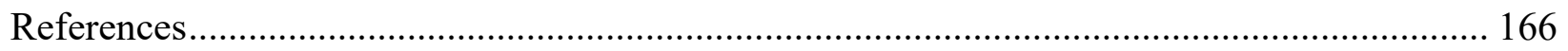

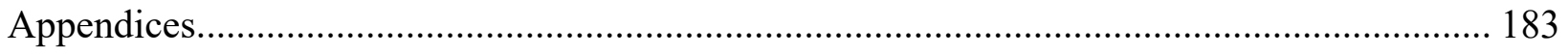




\section{LIST OF TABLES}

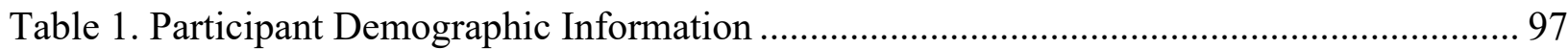




\section{CHAPTER ONE - INTRODUCTION}

"Death, especially in childhood, is conceptualized as a medical 'failure' and hospitals as a place for achieving 'survival"” (De Graves \& Aranda, 2005, p. 132). When young patients die from cancer, the nurses who strive to achieve patient survival experience intense feelings of grief but have limited time during their shifts to address a child's death and their feelings (Altounji, Morgan, Grover, Daldumyan, \& Secola, 2012; Conte, 2011; Lazzarin, Biondi, \& Di Mauro, 2012; Macpherson, 2008; Papadatou, 2001; Papadatou, Bellali, Papazoglou, \& Petraki, 2002; Reid, 2013). When nurses cannot attend to their feelings, they "often experience negative consequences of grief" (Conte, 2011, p. 93), which include "stress, fatigue, depression, anxiety, and disillusionment" (p. 93). According to Conte (2011), pediatric oncology nurses "experience multiple types of losses and grief" (p. 93), for example, "when a patient successfully completes treatment, fails treatment, or dies" (p. 93). Losing patients and not making time to process feelings of grief can be detrimental to the psychological wellbeing of this specialized group of nurses (Conte, 2011).

Pediatric oncology nurses, who are not supported or inadequately cope with their feelings of grief throughout their careers, may experience feelings of burnout and emotional exhaustion (Edmonds, Lockwood, Bezjak, \& Nyhof-Young, 2012; Pearson, 2013). Pearson (2013) stated the experiences of children's cancer nurses illuminated the "importance of having time to deal with their own emotions either through clinical reflection or debriefs within the multidisciplinary team" (p. 199). Making time to use self-reflective practices is recommended to enable nurses to cope with their feelings of distress and to thoughtfully examine how their behaviors can have lasting effects on their wellbeing and self-care practices (Koshy, Limb, Gundogan, Whitehurst, \& Jafree, 2017). Ruth-Sahd (2003) suggested, "Reflective practice is a means of self- 
examination that involves looking back over what has happened in practice in an effort to improve or encourage professional growth" (p. 488). For nursing professionals, self-reflection means

focusing on how they interact with their colleagues and with the environment to obtain a clearer picture of their own behavior. It is therefore a process by which practitioners can better understand themselves in order to be able to build on existing strengths and take appropriate future action. (Oluwatoyin, 2015, p. 28)

Understanding how pediatric oncology nurses effectively practice self-reflection is important because nurses learn how to care for dying patients and must also learn to adapt to the inevitable stressors associated with the workplace (Boyle \& Bush, 2018; Conte, 2011).

Nurses are a specialized group of professionals who have been "socialized to care for others and thus often prioritize their needs as second" (Halm, 2017, p. 344). According to Barnard, Street, and Love (2006), when nurses who specialize in cancer care cannot fully attend to their personal needs, workplace stress can be overwhelming. Workplace stressors specific to these nurses are "grief, loss, bereavement, moral, and ethical dilemmas regarding treatment decisions, the influence of clinical trials, complex treatment regimens, and managing professional boundaries in regard to relationships with patients and their families" (Zander, Hutton, \& King, 2010, p. 94). Yet, little is known about how self-reflective practices are used to manage workplace stressors. In a 12-hour shift, pediatric oncology nurses are responsible for providing timely and accurate treatments to patients and emotional support for their families. To fulfill these responsibilities, nurses spend nearly every minute of every workday focusing on performing medical tasks specific to patients. 
To effectively discuss the difficulties of death with children and their families, pediatric oncology nurses often suppress their feelings (Duke \& Appleton, 2000; Mann, Gordon, \& MacLeod, 2009). As time progresses, nurses learn and develop medical proficiency by spending most of their days interacting with patients and providing them with long-term care (Enskär, 2012; Hines \& Gaughan, 2014). Consequently, pediatric oncology nurses may develop close bonds with their patients throughout the extended treatment process (Hartlage, 2012). Caring for critical patients can be a source of worry, and dealing with emotions is often difficult for nurses because of the type of niche care pediatric oncology nurses provide to young children (Boyle \& Bush, 2018; Hartlage, 2012).

Nurses in pediatric oncology units may experience powerful emotions when the children they care for are suffering from pain or dying (Conte, 2014; Lazzarin et al., 2012; Macpherson, 2008; Papadatou et al., 2002). These nurses may be highly educated and spend years caring for young patients and responding to medical emergencies, but experiencing traumatic events can increase the emotional and physical stress they feel (Boyle \& Bush, 2018). Papadatou et al. (2002) reported after a child's death, pediatric oncology nurses could use strategies such as open expression of grief, acknowledgment of the child's death, peer support, and a sense of being valued to facilitate adjustment. Researchers have shown, when nurses are supported through lifealtering events, they can potentially minimize the harmful effects of stress and grief after a child has died (Conte, 2011, 2014; Davis, Lind, \& Sorensen, 2013; Papadatou et al., 2002).

Conversely, if nurses are not adequately supported, harmful feelings intensify over time (Barnard et al., 2006; Bowden et al., 2015; Chang, Kicis, \& Sangha, 2007; Davis et al., 2013; Emery, 1993; Emold, Schneider, Meller, \& Yagil, 2011; Gallagher \& Gormley, 2009; Hecktman, 2012; Kushnir, Rabin, \& Azulai, 1997; C. F. Morrison \& Morris, 2017). 
Pediatric oncology nurses receive emotional support from their peers, psychologists, and educational lectures (dos Santos et al., 2017). Additional supportive practices can be used when pediatric oncology nurses are provided with adequate time for self-reflection (Altounji et al., 2012). However, Hecktman (2012) reported, "Pediatric oncology nurses often care for high acuity patients with terminal diagnosis creating an environment with minimal time for selfrefection" (p. 356). Although little time is available for self-reflection, it is still a valuable skill for nursing professionals (Oluwatoyin, 2015). Self-reflection has been described as a unique talent, a method for enhancing learning and communication skills by asking the right questions, and a journey of self-discovery and self-transformation (Barbour, 2013; Dolphin, 2013; van Stralen, 2002).

Furthermore, ongoing self-reflection has been considered a method that helps nurses develop personally and professionally, which translates into better care for patients (Caldwell \& Grobbel, 2013; Gustafsson \& Fagerberg, 2004). Caldwell and Grobbel (2013) stated, "The dayto-day emotional impact of nursing can take a toll on nurses. Reflective practice allows nurses to have a safe outlet to discuss and better understand their feelings and practice" (p. 321). Although processing thoughts and emotions is essential for nurses (Conte, 2011), little is known about how pediatric oncology nurses with at least 10 years of experience use self-reflective practices in the clinical setting to cope with their feelings of stress and grief. According to Conte (2011), the experiences of grief among pediatric oncology nurses are considerably complicated because there are many factors to consider ... experience level of the nurse, the nurse's history of personal loss, the type of relationship the nurse had with the patient, the length of the relationship, and the circumstances surrounding the treatment of the patient. (p. 94) 
Grieving nurses must be supported, or the care they provide to patients may be negatively impacted (Conte, 2011).

Self-reflection is a skill that can be used by grieving nurses after the prolonged illness and death of young patients (dos Santos et al., 2017; Rashotte, Fothergill-Bourbonnais, \& Chamberlain, 1997). Though self-reflective practices are valued in nursing education (RuthSahd, 2003), what remains unknown is why and how experienced nurses use self-reflection throughout their careers. Pediatric oncology nurses, with years of clinical experience, are valuable resources for nursing students and novice nurses (Barnard et al., 2006). Barnard et al. (2006) stated:

The future of cancer nursing services is linked to the recruitment of newcomers to the profession, and also to the retention of experienced and qualified cancer nurses who are happy and satisfied in their vocation-these are the nurses who can provide high-quality nursing care to patients with cancer. (p. 341)

Researchers have reported experienced nurses (a) significantly support novice nurses throughout their clinical education as preceptors and mentors (Charalambous \& Kaite, 2013; Linder, 2009); (b) have greater capacity to cope with the demands of their setting (Hinds, Quargnenti, Hickey, \& Mangum, 1994; Pye, 2013); (c) report communication as an effective tool for delivering quality patient care (Franca, Costa, Lopez, Nobrega, \& Franca, 2013); and (d) improve their overall medical knowledge and intuition of patient care by learning from their past experiences (Dunn, Otten, \& Stephens, 2005). It remains unclear, however, the extent to which oncology nurses engage in self-reflective behavior and, if they do, manage to find time to use selfreflective practices effectively. 
In pediatric oncology work, experienced nurses are valuable resources to patients, families, and other nursing professionals (Charalambous \& Kaite, 2013; Linder, 2009; Montgomery, Sawin, \& Hendricks-Ferguson, 2017). Moreover, experienced nurses who make time for using self-reflective practices throughout their careers may improve patient outcomes while developing effective coping mechanisms to mitigate harmful feelings of stress, grief, and burnout (Altounji et al., 2012; Hinds et al., 1994; Pye, 2013). Retaining experienced pediatric oncology nurses who are happy, healthy, and satisfied is not only beneficial for the medical community, it is essential for recruiting, teaching, and developing valuable resources for new nurses interested in having a lengthy career in pediatric oncology (Barnard et al., 2006).

\section{Background}

Reflection is described as essential to nursing practice but not necessarily something nurses must use to provide care to their patients (Gustafsson \& Fagerberg, 2004; Oluwatoyin, 2015). In the literature, researchers have suggested pediatric oncology nurses are familiar with using self-reflective practices in two distinct ways: (a) to learn how to improve the art and science of nursing practice and (b) to make sense of unexpected events that may leave nurses feeling emotionally drained (Altounji et al., 2012; Charalambous \& Kaite, 2013; Enskär, 2012; Hines \& Gaughan, 2014; Jestico \& Finlay, 2017; Linder, 2009; Mirlashari, Warnock, \& Jahanbani, 2017). For example, Linder (2009) explored the experiences of pediatric oncology nurses during their first years of hire. Participants described using self-reflective practices as part of their orientation experiences and their professional growth and development (Linder, 2009). Altounji et al. (2012) described a self-care retreat for pediatric oncology nurses where reflective practice was used to help nurses cope with the demands of the workplace. Currently, there does not appear to be extensive quantitative studies that measure how using self-reflection reduces the 
effects of workplace stress or improves both personal and professional development of pediatric oncology nurses.

\section{Reflection and the Role of Expert Nurses}

The process of reflection is a learning process nurses use to understand how to enhance their clinical practice (Oluwatoyin, 2015). Reflection can be regarded as integral to transforming clinical knowledge into expert knowledge (Enskär, 2012). Enskär (2012) explored pediatric oncology nurses' perceptions of the role of an expert nurse. Participants in this study had between 6 and 30 years of clinical experience and were asked to write narrative responses to the question, "What does being an expert nurse in pediatric oncology mean to you?" (Enskär, 2012, p. 153). Results of this study were arranged in three categories: (a) an expert has confidence in their knowledge, (b) can provide high-quality care, and (c) takes opportunities for professional growth (Enskär, 2012).

Participants expressed the importance of using self-reflection to merge theory and practice and acknowledged a need to schedule time for reflection, either privately or with their peers. Additionally, participants in Enskär's (2012) study acknowledged time is a valuable commodity in the clinical setting. Novice nurses may not have the time or energy to spend reflecting on their patient and patient family interactions. However, according to participants, expert nurses were confident in their clinical knowledge and found time to reflect on how they could connect or empathize with patients and their families (Enskär, 2012). The results of Enskär's (2012) study are subtle regarding self-reflective practices, but the results point to the transformative nature of reflective practices for nurses. 


\section{The Art of Caring and Reflective Narratives}

Self-reflection is not only useful for nurses to bridge the gap between theory and clinical practice, but with experience, nurses can use self-reflection in their patient interactions (Enskär, 2012). Although performing medical tasks is critical to providing patients with quality care, Hines and Gaughan (2014) reported, "Caring within nursing practice exemplifies the hidden, intangible, unreimbursed, and often unrecognized work of nurses" (p. 26). Nursing practice may remain hidden until nurses reflect on their personal experiences to discover how their actions affected young patients (Oluwatoyin, 2015). To explore the hidden nature of caring, Hines and Gaughan (2014) stated, "Reflective narrative can be used to capture the essence of caring" (p. 27).

Hines and Gaughan (2014) studied nine nurse participants from a variety of pediatric practice settings with 10 or more years of clinical experience. Participants were asked to "reflect on a time in their practice that captured the essence of caring" (Hines \& Gaughan, 2014, p. 27), and participants suggested caring goes beyond performing medical tasks and is a feeling or sense of being connected and intimately present with patients. According to Hines and Gaughan (2014), "Nurses in this study described how caring within practice and a reflection on a caring encounter provided them with an inspiration for future practice” (p. 32). As previously stated, researchers have demonstrated forming relationships with patients can be a source of workplace stress for pediatric oncology nurses (Boyle \& Bush, 2018). However, using self-reflection seems to support a reduction of workplace stress for pediatric oncology nurses by helping them process stressful emotions and enable them to positively impact the lives of patients and their families (Enskär, 2012; Hines \& Gaughan, 2014). 


\section{Reflection and Managing Emotions}

Self-reflection has been associated with nurses' ability to manage and process the emotional demands of the workplace (Altounji et al., 2012; Charalambous \& Kaite, 2013; Jestico \& Finlay, 2017; Linder, 2009; Mirlashari et al., 2017). Altounji et al. (2012) described the development and implementation of self-care retreats (three total throughout the year), which were specifically tailored for pediatric oncology nurses to help them address a type of workplace stressor, moral distress. Moral distress is a feeling nurses experience that can cause "anger, frustration, guilt, loss of self-worth, depression, nightmares, suffering, resentment, sorrow, anxiety, helplessness, and powerlessness" (Lazzarin et al., 2012, p. 184). Because of the harmful effects of moral distress, the goal in developing and implementing self-care retreats was to allow pediatric oncology nurses time to rest, reflect, and heal from experiencing trauma (Altounji et al., 2012).

\section{Reflection and Managing Moral Distress}

Nurses appreciate the unique aspect of their work - caring for each patient (Hines \& Gaughan, 2014). With each distinct patient care experience, nurses may either cope well with their feelings or need extra time away from the clinical setting to address unresolved feelings of stress (Altounji et al., 2012). Pediatric oncology nurses in Altounji et al.’s (2012) study were asked to (a) describe their experiences, (b) address how to cope with trauma, and (c) discuss nursing empowerment and ways to achieve rest and relaxation. The aim was to "create a therapeutic and supportive environment for all participants, while allowing time for relaxation, reflection, and serenity" (Altounji et al., 2012, p. 19). While self-reflection is not directly addressed, the authors revealed reflective practice can be used as a method of self-care to decrease feelings of moral distress. 


\section{Student Nurses and Self-Reflective Diaries or Journals}

Due to positive benefits of using self-reflection (e.g., self-care), nursing educators have found value in teaching student nurses about reflective learning early in their careers. For example, self-reflection can be used as a teaching device to help professional nursing students cope with their clinical experiences. Charalambous and Kaite (2013) developed a study "to interpret the students' experiences of coming face-to-face with cancer care during their clinical placements" (p. 1). Participants in this study were fourth-year nursing students who were "practicing at oncology, hematology, pediatric oncology departments, and hospices" (Charalambous \& Kaite, 2013, p. 3). Self-reflective diaries were the main pieces of data collected; students were asked to record their experience during their clinical practice (twice a week for 6 weeks). Content analysis revealed seven themes. The fourth theme was "being selfreflective" where students "evaluated their own abilities, behaviors, and reactions towards the patients" (Charalambous \& Kaite, 2013, p. 6). When participants were being self-reflective, they recognized a spectrum of positive and negative aspects of providing care to cancer patients.

Like Charalambous and Kaite (2013), Mirlashari et al. (2017) developed a qualitative study to explore the perceptions of senior undergraduate nursing students. The aim of the study was to use self-reflective journals to understand the individual experiences of students and what it was like to care for children with cancer (Mirlashari et al., 2017). Students revealed three common themes in their self-reflective journals: (a) experiencing a state of shock and feeling lost, (b) walking into a mind-shaking world, and (c) finding the way (Mirlashari et al., 2017). Mirlashari et al. (2017) stated, "It seems that reflection and journaling enabled the students to gain a sense of usefulness, and this likely heightened their desire to request spending more time on the unit” (p. 26). These findings were similar to those of Altounji et al. (2012), Enskär (2012), 
Hines and Gaughan (2014), and Linder (2009), where nursing professionals used self-reflective practices to have a platform to liven their passion for the profession. Furthermore, student nurses who learn from their experiences and who use self-reflective practices may develop personal resilience that can help them cope with the demands of their profession (Jestico \& Finlay, 2017).

\section{Preparing Nurses to Cope With Workplace Stressors}

While reflective narratives, diaries, and journals are helpful for nurses to understand the process of self-reflection, Jestico and Finlay (2017) explored the perceptions of pediatric oncology nurses on whether their nursing education prepared them to care for children with cancer. Six nurses were recruited and interviewed. Thematic analysis conducted on the interview transcripts revealed three themes: (a) learning theory and practice, (b) caring for the child and family, and (c) developing resilience (Jestico \& Finlay, 2017). Due to the considerable number of stressful situations pediatric oncology nurses endured, nurses who are resilient and use selfreflection were more able to develop successful coping methods. According to Jestico and Finlay (2017), reflection was an integral aspect of the development of coping mechanisms and, thus, was useful for nurses learning how to manage workplace stressors. During their education and careers, nurses who explore their feelings of workplace stress may develop into more emotionally competent nurses (Caldwell \& Grobbel, 2013).

\section{The Use of Self-Reflective Practices}

In their literature review, Mann et al. (2009) found nurses who use self-reflection have the potential to improve patient outcomes. Further research is needed to address the nature of self-reflection and its effect on nursing practice. Self-reflection may be integral to the development of nursing practice by helping nurses integrate knowledge into practice (Caldwell

\& Grobbel, 2013; Cantrell, 2007; Enskär, 2012; Gustafsson \& Fagerberg, 2004; Hartlage; 2012; 
Hines \& Gaughan, 2014). My review of the literature revealed in pediatric oncology work, selfreflective practices are (a) essential to nursing professionals (Linder, 2009), (b) used by experienced nurses to reflect on patient interactions (Enskär, 2012), (c) used to discover the art of caring (Hines \& Gaughan, 2014), (d) used to manage emotions and cope with workplace stressors (Altounji et al., 2012; Jestico \& Finlay, 2017), and (e) used by nursing students to cope with their clinical experiences (Charalambous \& Kaite, 2013; Mirlashari et al., 2017). What remains unclear is how nurses use self-reflective practices in the pediatric oncology setting and how they develop individual practices that enhance coping throughout a lengthy career.

\section{Reflection and Emotional Wellbeing}

Pediatric oncology nurses are learning how to care for their emotional wellbeing by using self-reflection (Altounji et al., 2012; Enskär, 2012). However, nurses who work with critical patients have little time to spend in self-reflection in the clinical environment (Altounji et al., 2012; Caldwell \& Grobbel, 2013). Busy clinical environments and settings where self-reflection is not reinforced can act as barriers to the use of reflective practices and can limit the amount of time pediatric oncology nurses spend addressing practical situations or emotional experiences (Caldwell \& Grobbel, 2013; Mann et al., 2009). Despite these disadvantages, pediatric oncology nurses are required to provide patients and their families with compassionate care. Compassion is defined "as having a deep awareness of the suffering of another accompanied by the desired to relive it" (van Zyl \& Noonan, 2018, p. 1192). Although nurses are committed to providing compassionate care, the emotional labor of the pediatric oncology setting has been linked to nurse stress and burnout (Edmonds et al., 2012). According to Pearson (2013), "Emotional labor occurs when nurses portray the emotions which they feel should be displayed in a given situation while suppressing their true emotions" (p. 191). Pediatric oncology nurses who suppress their 
true emotions can develop feelings of burnout and lack of sympathy, even though they display behaviors of compassion toward their patients. Studies show nurses experience a negative effect from emotional labor; however, using self-reflective practices with colleagues has the potential to reduce the damaging effects of stress and the resulting burnout (Pearson, 2013).

\section{Statement of the Problem}

Pediatric oncology nurses have minimal time for self-reflection and self-care in the clinical setting (Altounji et al., 2012; Caldwell \& Grobbel, 2013; Enskär, 2012; Hecktman, 2012). Boyd and Fales (1983) described how medical situations can trigger nurses to use selfreflection. Even if an event triggers a nurse to use self-reflective practices, very little is known about the actual experience and how beneficial or detrimental reflection could be for pediatric oncology nurses. In a 12-hour shift, pediatric oncology nurses move around the unit, perform daily tasks, respond to medical emergencies, cover for other nurses, check monitors, listen to family concerns, and pause to embrace moments that feel meaningful (Wilson, 2005). Events can be sources of workplace stress (Gallagher \& Gormley, 2009). Workplace demands can limit the amount of time nurses spend on self-reflection and self-care (Boyle \& Bush, 2018).

\section{Nurses' Needs Come Second to the Patients' Needs}

Studies show nurses who do not address their feelings in a healthful manner may experience overwhelming stress (Barnard et al., 2006; Bowden et al., 2015; Chang et al., 2007; Davis, 2013; Emery, 1993; Emold et al., 2011; Gallagher \& Gormley, 2009; Hecktman, 2012; Kushnir et al., 1997; C. F. Morrison \& Morris, 2017). Barnard et al. (2006) said, "Stress is said to occur when the demands of one's work environment overwhelms [sic] one's coping resources" (p. 339). Yet, navigating the demands of the workplace setting is an essential element of providing accurate cancer treatments to critical patients. Pediatric oncology units are open 24 
hours a day. This reality means every hour of a nurse's shift is scheduled to ensure accurate and timely care. While unexpected events may occur during the shift, nurses are provided with set schedules so they can have a general idea about what to expect when they arrive on the unit. Nurses, whether they are experiencing overwhelming stress or not, have difficulty finding enough time during or between shifts to use coping strategies (Boyle \& Bush, 2018). Pediatric oncology nurses are continuously faced with the reality of the clinical environment, where their needs come second to the needs of their patients.

\section{No Time for Self-Care Leads to Stress and Burnout}

If the stress nurses experience continues to go unnoticed or remains unresolved, they may experience feelings of burnout. Burnout is a condition in which a nurse experiences "emotional exhaustion, depersonalization, and diminished feelings of personal accomplishments ... [and] may be associated with serious psychiatric comorbidity, including major depressive disorder and even suicide" (Moody et al., 2013, p. 275). Burnout is a hazardous condition and remains highly prevalent among pediatric oncology nurses (Barnard et al., 2006; Davis et al., 2013; Emold et al., 2011; Gallagher \& Gormley, 2009; Moody et al., 2013; C. F. Morrison \& Morris, 2017; Zadeh, Gamba, Hudson, \& Wiener, 2012). Stress and feeling burnt out can result in nurses leaving the profession (Davis et al., 2013).

Supporting pediatric oncology nurses in managing their feelings may have a direct impact on improving patient outcomes (Barnard et al., 2006). Altounji et al. (2012) reported, "Hospitals provide varying options for managing staff grief and burnout, including critical incident debriefings, support groups, yoga and meditation classes, and individual counseling through employee assistance programs" (p. 18). Due to the high levels of burnout associated with this profession, medical institutions continue to devise methods that ameliorate stress and provide 
emotional support for nurses (Edmonds et al., 2012). To ensure patients receive quality care, the emotional health of pediatric oncology nurses should be considered integral to the health of the children for whom they care (Boyle \& Bush, 2018). For these nurses to provide care to critical patients, they must manage their emotions in a healthful manner.

\section{Nurses Need Time for Self-Reflection}

Pediatric oncology nurses need adequate time to use self-reflective practices authentically and to rest from performing medical tasks (Boyle \& Bush, 2018; Zander et al., 2010; Zander, Hutton, \& King, 2013). When nurses make time for self-reflective practices, they can process situations with deep insight and empathy, find solutions to problems, engage in critical thinking, and develop resilience over time (Enskär, 2012; Robert \& Petersen, 2013). Experienced pediatric oncology nurses who have time to rest and reflect during their 12-hour shifts or after experiencing traumatic events may learn to let go of unresolved feelings that impede their ability to provide quality patient care (Altounji et al., 2012; Linder, 2009). According to Carlsson, Drew, Dahlberg, and Lützen (2002), “Expert care develops with reflection on theoretical knowledge in conjunction with the events and situations in which that knowledge is used. Eventually, clinical expertise becomes second nature, performed straightforwardly, without critical reflection on the basis of our acts" (p. 145). Carlsson et al. (2002) and Enskär (2012) agreed experience alone does not transform an experienced nurse into an expert-level nurse. Expert levels can be achieved when nurses use self-reflection as an ongoing process that assimilates theory and practice throughout nurses' careers (Boyle \& Bush, 2018; Mann et al., 2009). 


\section{Reflection and Experienced Pediatric Oncology Nurses}

Altounji et al. (2012), Charalambous and Kaite (2013), Jestico and Finlay (2017), Linder (2009), and Mirlashari et al. (2017) associated self-reflection with a nurse's ability to manage the emotional demands of the workplace. The use of self-reflective practices as an ongoing practice may help nurses relieve stress, which helps nurses develop professionally. Currently, there is little evidence to determine why experienced pediatric oncology nurses are reflecting or to ascertain the type of learning that results from their personal reflections. A deeper understanding of how experienced pediatric oncology nurses develop self-care practices and descriptions of their unique experiences with self-reflective practices are necessary. Awareness of the self is an important skill for nurses to apply in practice. Self-awareness and self-reflection may help pediatric oncology nurses sustain healthy minds during stressful and emotionally intense moments (Halm, 2017; Kushnir et al., 1997; Linder, 2009).

\section{Summary of the Problem}

Pediatric oncology nurses provide critical care to young patients from initial diagnosis, remissions, and death using the art and science of nursing practice (Boyle \& Bush, 2018; Cantrell, 2007; Hines \& Gaughan, 2014). Cantrell (2007) stated:

Even in the delivery of complex care interventions, such as implementing an intricate treatment chemotherapy protocol that requires a significant amount of scientific knowledge and critical thinking, nurses' creativity, resourcefulness, and imagination are required ... The nature of how a nurse implements a treatment protocol varies from individual to individual, and this knowledge comes only from his or her previous interactions with the adolescents and from his or her own intuition, reflection, and empathy. (p. 135) 
In this critical environment, unexpected experiences of heartbreak and tragedy produce extreme emotions, and nurses must be prepared to handle any situation by learning from their experiences and using self-reflective practices (Conte, 2011; Lazzarin et al., 2012; Macpherson, 2008;

Oluwatoyin, 2015; Papadatou et al., 2002). As time progresses, pediatric oncology nurses may neglect to process their emotions in a healthful manner. One of the reasons for this neglect is the lack of time to address activities that serve their best interests. Studies show nurses value their workplace relationships and continuously attend to the needs of their patients; however, these work interactions and care duties leave little time for nurses to think and process their emotions in an effective manner (Forsey, Salmon, Eden, \& Young, 2013).

\section{Addressing the Problem}

Understanding how experienced pediatric oncology nurses use self-reflective practices may help address the problem of workplace stress and feelings of burnout. To better understand how a nurse's mind is developed through self-reflection, researchers must begin by exploring the direct experiences of nursing professionals (Boyd \& Fales, 1983). Self-reflective practices are related to professional development and coping strategies; however, there is a belief little to no education or professional development is tailored to teaching nurses how to cope with the death and suffering of their patients (Gallagher \& Gormley, 2009). Therefore, my goal with this qualitative research was to understand the experiences of nurses who already use self-reflective practices and to uncover thoughts and behavioral processes that enhance self-care abilities.

\section{Purpose of the Study}

Nurses are using self-reflection more and more over time (Barbour, 2013; Enskär, 2012;

Oluwatoyin, 2015; Schön, 1983). Beckstrand, Rawle, Callister, and Mandleco (2010) stated: 
Although improved communication has been recommended for better end-of-life care, nurses also must be aware of their own values, understanding, and comfort levels with the language of death, realizing that some children in their care will still die despite everyone's best efforts. (p. 546)

This experience of loss can be emotionally and physically draining for nurses who directly cared for critical patients. Revealing how experienced pediatric oncology nurses use self-reflection may fill the gap in the literature. Researchers may gain a better understanding of how nurses experience and combat the effects of stress and how they learn, develop, and cope while working in the clinical setting.

Levels of experience and expertise, although important for developing clinical skills, do not determine how a nurse experiences and copes with workplace stress (Bowden et al., 2015; Davis et al., 2013; Morrison \& Morris, 2017). Limited research exists on how pediatric oncology nurses develop expertise when they are continuously exposed to intense grief, moral distress, feelings of helplessness, personal and professional stress, and emotional and physical burnout. Learning how these specialized nurses describe their unique patient care experiences may show how they self-reflect and cope with the emotional and physical demands of their profession. Therefore, my purpose for this study was to explore the experiences of pediatric oncology nurses with 10 or more years of bedside experience who identify with using self-reflective practices in their daily lives.

\section{Research Question}

I used Husserl's (1931) transcendental (descriptive) phenomenology (TP) as the theoretical framework. Transcendental phenomenology is used to describe the essence of lived experience by exploring a structure of consciousness known as intentionality (Moustakas, 1994). 
More information on this theoretical framework is described in Chapter 3. Because of the need to obtain specific information to inform this study, the use of in-depth interviews was selected to systematically address the primary research question (Moustakas, 1994): What is the lived experience of expert-level pediatric oncology nurses using self-reflective practices in patient care?

\section{Methods Used to Address the Research Question}

According to Husserl (1931), learning something new by setting aside prejudgments about the phenomenon of self-reflective practices and about the experiences of pediatric oncology nurses would be considered transcendental (Moustakas, 1994). Nurses in this study were asked to describe experiences when they used self-reflection and the meaning of selfreflection whether in a positive or negative context. To help frame the interview questions, Schön's (1983) reflection-on-action theory was used. According to Burns and Bulman (2000):

Reflection on action is the retrospective contemplation of practice in order to uncover the knowledge used in a particular situation, by analyzing and interpreting the information recalled. The reflective practitioner may speculate how the situation might have been handled differently and what other knowledge would have been helpful. (p. 5)

Although nurses may use self-reflective practices in the process of care (reflection-in-action), participants in this study were asked to reflect back on their experiences (Schön, 1983). The main objectives of this study were threefold: (a) to explore experienced pediatric oncology nurses' descriptions of how they developed an understanding of reflective practices in patient care, (b) to explore their experiences of what self-reflective practices means to them in the clinical setting, and (c) to explore their descriptions of using self-reflective practices and the potential impact it may have on their wellbeing and professional longevity. 


\section{Definitions of Terms}

Moustakas (1994) stated, "The key words of the [phenomenological] question should be defined, discussed, and clarified so that the intent and purpose of the investigation are evident" (p. 104). The key words or phrases used in this study are defined to best clarify their specific meanings. These definitions were provided to each participant via the consent form.

Experienced pediatric oncology nurses are nurses who identify with using self-reflective practices in the clinical setting and who have been employed for 10 or more years providing care to pediatric inpatients and outpatients diagnosed with cancer-related disorders.

The term lived experiences is defined using phenomenological terminology. According to van Manen (2014):

Phenomenology does not study the "what" of our experience but the "experience" of the what - the experience of the intentional object, thing, entity, and event as it appears in consciousness. Phenomenology is the study of phenomena, and the phenomena are someone's experiences_-belonging to someone's stream of consciousness . . . experience is the thing and "how" the things of experience appear to consciousness is the focus. (p. 91)

The description of lived experience by pediatric oncology nurses is the what in phenomenological research and how self-reflective practices appear to these nurses is the phenomenon in question.

To create the phrase self-reflective practices, the word self was added to the definition of reflective practice, according to Boyd and Fales (1983). Reflection, reflective practice, and selfreflective practices are not easily distinguishable in nursing literature. Self-reflective practices are considered a type of learning strategy that promotes and develops self-awareness (Boyd \& 
Fales, 1983; Oluwatoyin, 2015). For this study, experienced pediatric oncology nurses were identified as using self-reflective practices in the context of learning about the self and learning the value of self and others in the process of care (Boyd \& Fales, 1983). Additionally, selfreflective practices are addressed as a process of reflecting back on past situations and striving to do something productive (Kelsey \& Hayes, 2015; Oluwatoyin, 2015; Schön, 1983).

Lastly, patient care is defined as the care provided to critical patients ranging from birth to age 21 who have been diagnosed with cancer-related disorders. Patient care is delivered in the clinical setting where nurses are responsible for working three 12-hour shifts and having roughly 90 minutes total for non-task activities (e.g., taking rest breaks, lunch) during a typical work week.

\section{Significance of the Study}

Keeping pediatric oncology nurses employed and developing methods that support them throughout a lengthy career has the potential to influence the quality of patient care (Benner, 2001; Hines \& Gaughan, 2014; Reid, 2013). In the years to come, the overall nursing shortage will greatly influence the delivery of acute and long-term patient care to children with cancer (Erikson, Salsberg, Forte, Bruinooge, \& Goldstein, 2007; Heller, Oros, \& Durney-Crowley, 2000; Linder, 2009). Nurses able to reflect on their past experiences may learn how to enhance their personal and professional critical thinking abilities and, potentially, obtain better patient care while remaining in pediatric oncology work (Benner, 2001; Chacko \& Sreerenjini, 2012; Dolphin, 2013; Fejes, 2008; Hines \& Gaughan, 2014; Kelsey \& Hayes, 2015). I explored the lived experiences of nurses with 10 or more years of experience and contributed knowledge about experienced nurses who identify with using self-reflective practices in the clinical setting. Supporting experienced nurses and learning how they use self-reflection may contribute to 
knowledge that helps other healthcare professionals understand how nurses in high-trauma careers emotionally heal and avoid burnout.

\section{Subsequent Contribution}

As a doctoral student and certified athletic trainer, I believe this study improved my understanding of how nurses in high trauma settings use self-reflection. Athletic trainers have “traditionally practiced interprofessionally, working with health professionals such as physicians, physical therapists, nurses, dieticians, and pharmacists. The athletic trainer maintains continuous contact with and advocates for the patient while engaging with these and other health professionals" (Rizzo, Breitbach, \& Richardson, 2015, p. 256). Athletic trainers collaborate with nurses on the safety and wellness of student-athletes in the secondary school setting (Geisler, 2015; Rains \& Robinson, 2010, 2012; Rizzo et al., 2015). Geisler (2015) stated, "I learned how to be an interprofessional collaborator by practice, by process, by reflecting and thinking, and, mostly, by doing" (p. 3). However, interprofessional education for both athletic training students and other health professions such as nursing is limited (Rizzo et al., 2015).

Athletic trainers also experience similar effects of workplace stress and burnout (DeFreese \& Mihalik, 2016). Currently, little is known about how athletic trainers and nursing professionals best address and cope with the harmful effects of stress and burnout. The caring profession has long been considered one where care is provided to patients but not to the caregiver. As a doctoral student, I aspire to teach students in higher education. Specifically, I intend to educate and support students who are interested in any of the caring professions. I want to generate evidence-based research to develop methods that teach future caregivers how to better cope with workplace stress that produces feelings of burnout. 


\section{Conclusion}

Understanding and appreciating the individual self-reflective practices experienced nurses use in patient care may lead to actual change (Boyd \& Fales, 1983) in the nursing profession and in the development of quality nurses able to provide more effective patient care. My aim with this study was to contribute to the understanding of the lived experience of using self-reflective practices among experienced pediatric oncology nurses employed in a teaching hospital in Southern California to address the lack of information pertaining to individual reflective practice in pediatric oncology nursing and to support the creation of reflective practice models in the context of self-care. Furthermore, I addressed a gap in the literature by asking nurses to reflect on patient care experiences and to describe the types of self-reflective practices used to manage their emotionally and physically challenging careers.

\section{Dissertation Format}

This dissertation is arranged in five chapters. Chapter 1 includes the background and contextual underpinnings that were the impetus to this qualitative inquiry. Chapter 1 also includes a section on the statement of the problem, purpose of the study, and significance of the study. A review of the literature, including a description of the philosophical and theoretical underpinnings of reflective practices, self-reflective practices in the context of pediatric oncology, and key areas of the literature that inform this phenomenological research is included in Chapter 2. A critique of the literature is addressed at the end of Chapter 2 along with an argument for developing this phenomenological investigation. Chapter 3 has a description of the philosophy and TP methodology and a discussion section for research ethics. I discuss the specific steps taken to address the research question in the methods section. A section on the trustworthiness of the study is also in Chapter 3. I detailed results of the study in Chapter 4 and 
identified two categories: (a) the reports of experienced pediatric oncology nurses using selfreflective practices in patient care and (b) the experience of using self-reflective practices away from the clinical setting. Specific quotations are provided as evidence. Chapter 5 contains the discussion section in which results of this study are discussed in detail and aligned with the current literature to add to the dimensions of self-reflective practices in the context of pediatric oncology. The limitations of the study are described after the detailed discussion of results. Implications for future research are addressed at the end of Chapter 5. 


\section{CHAPTER TWO - REVIEW OF LITERATURE}

In accordance with the methodology of descriptive phenomenology outlined in Chapter 3, I conducted an initial review of the literature pertaining to the motivation, circumstances, and willingness of nurses to use self-reflective practices in pediatric oncology nursing. I explored concepts, theories, and models that provided a structure and guide for understanding what reflective practices occur in nursing practice. I used the literature review to inform this study as (a) a guide to learn about self-reflection, (b) a review of literature on the reflective practice experiences of nursing students and experienced nurses, and (c) a vehicle to address the gap in the literature on pediatric oncology nurses and their experiences using self-reflection.

Limited information exists on how pediatric oncology nurses use self-reflection to learn in the clinical setting or how they cope with their emotions (Altounji et al., 2012; Caldwell \& Grobbel, 2013; Mann et al., 2009; Ruth-Sahd, 2003). Nursing educators and nursing professionals are "encouraged to engage in or promote reflective practice in their students but are shown very little evidence that it actually improves practice or results in learning" (Ruth-Sahd, 2003, p. 488). I use Chapter 2 to overview the framework to support the research question: What is the lived experience of expert-level pediatric oncology nurses using self-reflective practices in patient care? Understanding the experiences of pediatric oncology nurses whose daily use of self-reflection in their nursing practice may reveal the type of learning that results from on-going self-reflection. My aim with Chapter 2 was to develop an understanding of self-reflective practices and to describe how using reflection in the nursing community results in learning.

I began my review of the literature for this study with a search of academic databases using the following key terms: self-reflective practices, reflection, nursing, nurses, education, and pediatric oncology. Much of the literature pertaining to nurses, especially to pediatric 
oncology nurses and to reflective practices, was published after the 1970s. Additionally, studies pertaining to nurses and their unique experiences using self-reflective practice did not emerge until the early 21st century (Gustafsson \& Fagerberg, 2004; Mann et al., 2009; Ruth-Sahd, 2003). Therefore, this review of literature begins in the 1970s and continues chronologically through present day. These sections reveal how self-reflective practice in broad terms has emerged as an educational tool for nursing professionals over the decades. Current studies are combined with studies from the 1970s, 1980s, and 1990s to strengthen the understanding of selfreflective practice in nursing. The following section begins with a general description of selfreflective practice (i.e., reflective thinking) and continues to more nurse-specific discussions of self-reflective practices.

\section{Theoretical Underpinnings of Reflective Practice in Nursing}

Nurses use reflective practice to thoughtfully address past events and to consider exploring feelings that can have a direct impact on patient outcomes (Boyd \& Fales, 1983). Researchers have agreed nurses learn by using self-reflection at the onset of their education and during every aspect of their personal and professional development (Mann et al., 2009; Ruth-Sahd, 2003). In theory, pediatric oncology nurses use self-reflective practices as a learning method for personal and professional development, as a coping strategy to assist with reflection on workplace stressors, and to address unresolved emotions (Altounji et al., 2012; Enskär, 2012). Yet, few have researchers addressed the value of reflective practices and how such practices result in learning and improved patient outcomes in the context of pediatric oncology nursing. Researchers must address the problem of what constitutes self-reflection because the process shares similar attributes with intuition, resilience, reflection-in-action, and tacit knowledge as nurses gain more and more clinical experiences (Boyle \& Bush, 2018; 
Carlsson et al., 2002; Schön, 1983). Self-reflection is a critical component of the overall development of a nurse, but how prevalent is the process? If self-reflection occurs, why and how do pediatric oncology nurses use self-reflective practices? Answering these questions requires extensive knowledge of what self-reflection is in broad terms and how it is used to learn and develop knowledge in the nursing profession.

Reflective practice for nurses is a method of processing past events to develop future understanding and meaning (Gustafsson \& Fagerberg, 2004). Reflection is used to understand the self in relation to others and to help nursing professionals learn how to integrate theory into practice (Peden-McAlpine, Tomlinson, Forneris, Genck, \& Meiers, 2005). Self-reflective practice for nursing professionals emerged as a popular concept in the mid-20th century (Enuku \& Evawoma-Enuku, 2013). According to Enuku and Evawoma-Enuku (2013), "Nursing as a profession has been regarded as a profession that concentrated upon action or doing since the 1950s and 1960s, which was passed on through the apprenticeship form of education apparent in the twentieth century" (p. 57). As the nursing profession continued to grow and evolve, educators and philosophers argued "the mind and body are interconnected and the knowledge is socially rather than individually constructed and thus thinking, feeling, and action are intertwined" (Enuku \& Evawoma-Enuku, 2013, p. 57). After the 1950s and 1960s, reflective practice was slowly integrated into nursing education (Mann et al., 2009; Oluwatoyin, 2015; Schön, 1983).

\section{Self-Reflective Practice in the 1970s}

Borton (1970) described a simple method of self-reflection that can be used in nursing education but that is not particularly meant for nursing professionals. Borton's (1970) method of self-reflective practice outlined three simple questions to stimulate reflective thinking: What? So 
what? Now what? People who use these questions are stimulated into reflective thinking (Borton, 1970). Self-reflection occurs when a person or groups consciously and purposefully explore an experience by using a stream of questions - that is, What happened? What was my role in this experience? What behaviors, thinking patterns, and emotions am I experiencing? In this process of reflective thinking, a person reviews questions and describes their experiences to better understand and analyze critical situations.

Although reflective practice can be accomplished by asking oneself questions, reflective thinking is not easily distinguishable from other forms of thinking (Boyd \& Fales, 1983; Mann et al., 2009; Ruth-Sahd, 2003; Schön, 1983). Moreover, the experience of using self-reflective practice is difficult to quantify because asking oneself questions, as Borton (1970) outlined, is an act of doing reflection as opposed to being reflective (i.e., as in continuously learning). Since nursing practice has been regarded as a process of doing (Enuku \& Evawoma-Enuku, 2013), it is not surprising reflection is not easily distinguishable from being reflective. Being reflective and transforming the mind is what some authors believe is necessary to improve learning and understanding (Boyd \& Fales, 1983). Learning from experience, changing perspective, and transforming behaviors may occur when nurses use reflective thinking in meaningful or purposeful manners (Boyd \& Fales, 1983; Fowler, 2014).

\section{A Process of Self-Reflective Practice in the 1980s}

Boyd and Fales (1983) argued reflective thinking occurs because everyone may already perform a type of self-reflective thinking without ever realizing it. Reflective practice is not merely asking questions; rather, it is an ongoing process of routinely questioning and analyzing one's thoughts to identify and change an approach to similar future events (Schön, 1983). Nonetheless, one must be careful not to misinterpret routine or habitual reflection with the type 
of purposeful self-reflection that leads to a transformation of perspective. Boyd and Fales (1983) stated:

The process of reflection is the core difference between whether a person repeats the same experience several times, becoming highly proficient at one behavior, or learns from experience in such a way that he or she is cognitively or affectively changed. Such a change involves essentially changing his or her meaning structures. (p. 100) Though nurses can use self-reflection in their daily lives, if the process is not meaningful to them, authentic learning may not develop (Fowler, 2014).

At the core of using reflective practice is a fundamental change in attitude and behavior (Oluwatoyin, 2015), and developing such practices occurs over years of implementation (Mann et al., 2009). Therefore, reflective thinking is useful for professional development when operatives are expected to learn from their experiences and change their behaviors accordingly (Schön, 1983). The value of using self-reflective practices was becoming well understood as a tool for professional development and ongoing learning in the 1970s and 1980s (Borton, 1970; Boyd \& Fales, 1983; Schön, 1983). These models of self-reflective practice are not specific to nursing but can be useful for helping nurses develop in professional roles.

Reflective practice model for professionals. Reflection can be a well-thought-out method of processing events in progress or past events (Oluwatoyin, 2015; Schön, 1983). For example, Schön's (1983) reflective practice model was developed for professional selfreflection: (a) reflection-in-action and (b) reflection-on-action. Reflection-in-action occurs in the moment, while reflection-on-action is a process of learning from experiences to develop knowledge and understanding. Self-reflective practice is considered a learning strategy to help 
develop self-understanding and awareness from experiences (Boyd \& Fales, 1983; Schön, 1983). Boyd and Fales (1983) described self-reflection

as the process of creating and clarifying the meaning of experiences (present or past) in terms of self (self in relations to self and self in relation to the world). The outcome of the process is changed perspective. The experience that is explored and examined to create meaning focuses around or embodies a concern of central importance to the self. (p. 101) In addition to Schön's model of professional self-reflective practice and Boyd and Fales' (1983) description of self-reflective learning, Kolb's (1984) experiential learning cycle was developed. Kolb's learning cycle contains four components: (a) the concrete experience, (b) the reflective observation, (c) the observation abstraction, and (d) the active experiment.

Reflective practice that leads to action. Self-reflective practices are thoughts that can be translated into action (Kolb, 1984). Being self-reflective is a way of learning and thinking about present or past events and viewing them differently (Schön, 1983). This technique is similar to the self-reflective model of Boud, Keogh, and Walker (1985), which "points to the starting point and objects of reflection: the totality of experiences of learners, the behavior in which they have engaged, the ideas of which learners are aware, and the feelings which they have experienced" (p. 20). For Boud et al. (1985), reflective practice is a systematic process of thinking and engaging, but Kolb's (1984) method of reflection is meant to inspire action. Learning that occurs from self-reflection is not passive or merely abstract because it involves a series of processes (i.e., thoughts, emotions, behaviors) that lead to action and change (Gibbs, 1988; Kolb, 1984).

A cycle of reflective practice. Gibbs (1988) developed a cycle of self-reflection based on Kolb's cycle. Gibbs used structured questions to help subjects reflect and analyze detailed 
situations in attempts to clarify specific learning outcomes. The six stages of Gibbs' cycle of reflection are (a) description of the event (What happened?), (b) feelings (What were you thinking and feeling?), (c) evaluation (What was good and bad about the experience?), (d) analysis (What sense can you make of the situation?), (e) conclusion (What else could you have done?), and (f) action plan (If the situation arose again, what would you do?). Reflective practices were developed and viewed as simple processes of reflecting on past events to change future behaviors in the 1970s. These practices were then transformed into complex thinking cycles that lead to changes in behavior in the 1980s (Gibbs, 1988; Kolb, 1984; Oluwatoyin, 2015; Schön, 1983). For example, nurses are faced with constantly evolving situations in which they are responsible for developing alternative courses of action without compromising the health of their patients (Boyle \& Bush, 2018). According to Oluwatoyin (2015), "The word 'action' is vital. Reflection is not 'navel-gazing.' The aim is to develop professional actions that are aligned with personal beliefs and values" (p. 28). Self-reflection is the act of examining how one thinks and feels to produce desired outcomes.

Educators and critical reflective practice. People who practice self-reflection most complete a critical assessment of past experiences because thoughts, beliefs, and values have the capacity to transform into meaningful actions (Mann et al., 2009). For example, Smyth (1989) moved from general methods of using self-reflection and a cycle of reflective practice (e.g., learning from experience and developing action) toward reflecting on experiences associated with power imbalances where individuals may feel disempowered to cause change. Smyth (1989) developed a type of critical reflection to empower teachers to affect change: (a) describe (What do I do?), (b) inform (What does this mean?), (c) confront (How did I come to be like this?), and (d) reconstruct (How might I do things differently?). Critical reflection may be used 
as a cycle of reflective questions for nursing students, nurses, and nursing educators and as a form of emancipatory (i.e., freeing) reflective practice that can cause change in the entire profession (Kelsey \& Hayes, 2015). The models previously discussed are not specific to the nursing profession but have been described in the literature as essential to helping nurses learn and develop in their professional roles (Mann et al., 2009; Oluwatoyin, 2015; Ruth-Sahd, 2003). The evolution and popularity of self-reflective practice as a learning tool for professional growth helped researchers begin to develop theories of reflective practice that can be specific to nurses and their unique workplace environment (Atkins \& Murphy, 1993).

\section{Reflective Practice in the 1990s}

In the 1990s, reflective practice was developed and refined as a set of skills to help nurses integrate theory and clinical practice with a focus was on how emotions play an important role in the overall learning process (Atkins \& Murphy, 1993). The Atkins and Murphy (1993) model of reflective practice for nursing professionals includes five cyclical stages: (a) being aware of uncomfortable feelings and thoughts (a beginning or end phase where an individual describes a new experience or develops an action plan); (b) describing situations including thoughts and feelings — salient events and key features; (c) analyzing feelings and knowledge relevant to the situation-identifying knowledge, challenging assumptions, and imagining and exploring alternatives; (d) evaluating the relevance of knowledge — determining if it helps to explain or solve problems; and (e) identifying any learning that has occurred. The reflective model of Atkins and Murphy (1993) may help nurses develop the necessary skills to incorporate reflective practices into patient care and to develop emotional competence that inspires a change in their behaviors. Nurses, especially nurse managers and leaders, must develop the necessary skills to use their emotions in a positive manner (Kerfoot, 1996). In nursing practice, the use of self- 
reflection and learning to process emotions in a positive manner is integral to providing quality care (Atkins \& Murphy, 1993).

\section{A Summary of Self-Reflective Practices From the 1970s Through the 1990s}

The reflective practices shared in the previous section are thoughtful processes of questioning, action-oriented approaches to learning from experience, processes that involve understanding emotions, and processes that may lead to self-awareness and changes in behavior (Atkins \& Murphy, 1993, 1994; Borton, 1970; Boud et al., 1985; Boyd \& Fales, 1983; Gibbs, 1988; Kolb, 1984; Schön, 1983; Smyth, 1989). However, only one model of reflective practice was presented that specifically pertains to the nursing profession (i.e., Atkins \& Murphy, 1993). Nursing scholars identify the other models mentioned (Borton, 1970; Boud et al., 1985; Boyd \& Fales, 1983; Gibbs, 1988; Kolb, 1984; Schön, 1983; Smyth, 1989) as integral to understanding how nurses can learn and develop by using self-reflection (Fowler, 2014; Mann et al., 2009; Ruth-Sahd, 2003). I initially conducted the literature review to understand self-reflection in the context of nursing practice. Learning about self-reflection in a chronological manner, however, is useful for identifying general models of reflective practice designed for nursing educators (i.e., Atkins and Murphy's [1993] model of reflective practice).

In the 1970s and 1980s, reflective practice presented an array of questions meant to provoke a change in perspective followed by action (Boud et al., 1985; Boyd \& Fales, 1983; Gibbs, 1988; Kolb, 1984; Schön, 1983; Smyth, 1989). Reflective practices were meant to help professionals learn and develop in complex environments. Educators were interested in reflective practice because it led to changes in behavior, which translated into effective actions (Gibbs, 1988; Kolb, 1984). Additionally, self-reflection was not a passive process but an active method of learning and developing from the inside out. In the 1990s, ideas of self-reflection continued 
evolving and scholars began to understand its effect on professionals and students. Specifically, self-reflection could be used to help professionals learn self-awareness and learn how emotions matter to learning. The self is central to reflective practice and to the concept knowledge is valuable and meaningful when the self is intimately understood in relation to others.

\section{Emotional Intelligence, Self-Awareness, and Self-Reflection}

In the 20th and 21st centuries, reflective practice continued to develop as a process of self-awareness by analyzing one's thoughts and emotions (Chacko \& Sreerenjini, 2012; Pool, 2018). For example, in the mid-1990s, nursing scholars and educators sought to improve patient care by recruiting nurses who were aware of their emotions and could process those emotions skillfully in the clinical setting (Kerfoot, 1996). In accordance with Atkins and Murphy's (1993) reflective model, Kerfoot (1996) stated being self-aware "is the most important aspect of emotional development and the key to emotional reaction and control" (p. 60). Popularized by Goleman in 1995, emotionally intelligent leaders are people who possess interpersonal intelligence (Kerfoot, 1996). According to Kerfoot (1996), being emotionally intelligent "consists of the ability to read and respond appropriately to the moods and emotions of others, the ability to monitor one's own feelings, and the ability to discriminate about those feelings and the appropriate responses to these emotions" (p. 59). Kerfoot (1996), a registered nurse and scholar, developed an article titled, "The Emotional Side of Leadership: The Nurse Manager's Challenge.” Kerfoot (1996) described Goleman's five aspects of emotional intelligence: “(a) knowing one's emotion, (b) managing emotions, (c) motivating oneself, (d) recognizing emotions in others, and (e) handling relationships" (p. 59). Being emotionally intelligent is being self-aware (Kerfoot, 1996), and self-awareness is a key aspect in developing self-reflective practices in nursing professionals (Hentz \& Lauterbach, 2005). 
Self-reflection as a leadership strategy. The nursing profession is expected to experience "a shortage of 400,000 registered nurses" (Shirley, 2006, p. 256) by the year 2020. Shirley (2006) stated, “The healthcare work environment as a source of stress has been implicated in the shortage of nurses" (p. 256). Shirley (2006) further explained, "Healthy work environments, guided by authentic leaders, produce superior outcomes for both staff nurses and patients" (p. 264). In leadership research, authentic leaders are defined as those who are deeply aware of how they think and behave and are perceived by others as being aware of their own and others' values/moral perspectives, knowledge, and strengths; aware of the context in which they operate; and who are confident, hopeful, optimistic, resilient, and of high moral character. (Avolio \& Gardner, 2005, p. 321)

Authentic leaders are "genuine, trustworthy, reliable, and believable" (Shirley, 2006, p. 259) who develop skills such as emotional intelligence (Akerjordet \& Severinsson, 2008) and who may play an important role in providing nurses with a safe and healthful workplace environment. Akerjordet and Severinsson (2008) stated:

Emotional intelligence cannot be considered a general panacea, but it may offer new ways of thinking and being for nurse leaders, as it takes the intelligence of feelings more seriously by continually reflecting, evaluating, and improving leadership and supervisory skills. (p. 565)

Nursing educators, authentic leaders, and staff can benefit from developing emotional intelligence and processing emotions by using self-reflective practices (Horton-Deutsch \& Sherwood, 2008). According to Horton-Deutsch and Sherwood (2008), "The remarkable global spread of reflection in nursing education, practice, and research follows an emphasis on developing self-awareness as a leadership strategy for improving individual and organizational 
performance" (p. 946). When nurses are educated to be self-reflective in their practice, they may develop into emotionally competent leaders (Horton-Deutsch \& Sherwood, 2008).

\section{Nursing and the Skills Required for Using Self-Reflective Practices}

In general, self-reflective practice is when a person focuses on understanding personal feelings and developing behaviors that encourage professional growth (Ruth-Sahd, 2003). Furthermore, professional development leads to "better nursing care for the patients" (Gustafsson \& Fagerberg, 2004, p. 271). Hentz and Lauterbach (2005) stated, "The model of self-reflection begins with creating a right relationship with self, a process [a form of praxis: reflectionawareness-action-reflection-awareness-action] that begins with a focus on personal experiences and meanings" (p. 26). In this manner, reflective practices are like Boyd and Fales' (1983) description of self-reflective learning, which has an emphasis on understanding the self in relation to the world. For nurses to provide better care to patients and to use self-reflective practices effectively, they must develop skills to do so.

McGrath and Higgins (2006) described reflective skills as "critical analysis, synthesis, evaluation, clinical reasoning skills, problem solving, and self-awareness" (p. 176). Robert and Petersen (2013) and Oluwatoyin (2015) described two additional skills required for reflective practice: description and critical thinking. Although researchers have identified reflective skills, according to McGrath and Higgins (2006), "The literature on how to teach and incorporate reflective sessions into one's teaching is limited" (p. 176). Two methods to teach nursing students reflective practices are introducing them to guided reflective practice (Bailey \& Graham, 2007) or encouraging them to participate in research activities that explore selfreflective practices (Chong, 2009; Rees, 2013). 
Nurses who work in clinical settings require adequate time for self-reflection because care and reflective thinking occur in unpredictable environments (Edwards, 2003). Reflective practice in the clinical setting can be a method nurses use to better understand themselves, but the process may not translate into quality patient care (Chacko \& Sreerenjini, 2012; Hentz \& Lauterbach, 2005; Oluwatoyin, 2015; Ruth-Sahd, 2003). However, Karbasi, Pacheco, Bull, Evanson, and Chaboyer (2018) argued nurses align their care with "a patient-centered approach" (p. 60) and "rely on reflective practices and on the support of others" (p. 60) to provide patientcentered care. In essence, the art of nursing practice begins with learning about oneself and being fully present emotionally with patients and their families (Cantrell, 2007).

\section{Nurses and Time Spent Using Reflective Practice}

Pediatric oncology nurses are required to learn science and the art of improving their patients' health (Cantrell, 2007). Pediatric oncology nurses provide care in an environment where

much of the required knowledge for the art of nursing practice becomes known to nurses through interactions with patients and families. To gain this knowledge, the nurse's practice must be intuitive, reflexive, and open to the adolescent's perception of the experience. (Cantrell, 2007, p. 135)

Studies show, however, not all nursing professionals use self-reflective practice in the clinical setting (Mann et al., 2009). Furthermore, for nurses who use self-reflection, the amount of time they may spend doing so in the clinical environment can vary (Mann et al., 2009). Fowler (2014) suggested, "Reflective practice is probably one of the most talked about but least practiced aspects of nursing” (p. 398). Fowler (2014) argued the most important rule of using reflective practice is to make time for it. 
When nurses find time in their shifts to use reflective practice, they "need to link that time to specific clinical practice or individual patient care" (Fowler, 2014, p. 398). There are barriers to using reflective practices in the clinical setting. A barrier nurses face daily is finding energy for using reflective practices during their shifts. According to Fowler (2014), "finding energy for reflection" (p. 398) can be a challenge for nurses working long shifts. Therefore, using reflective practices is, at times, not possible even when nurses "want to do it" (Fowler, 2014, p. 398). Though Fowler (2014) highlighted the possibility of nurses using reflective practice, the current research is limited on descriptions of why nurses use it and how effective it is in the clinical setting. A reason for this lack of research may be the barriers that impede nurses from using self-reflective practice.

\section{Barriers Nurses Encounter When Using Reflective Practice}

Barriers to using reflective practice in the clinical setting include (a) the time required, (b) the lack of organizational structure to support reflection, and (c) the nurses' perceptions that reflective practices are based on impractical, theoretical approaches to solving real life problems (Mann et al., 2009). Reflective practices can be used to help nurses learn from past experiences and to help them change future behaviors (Mann et al., 2009; Pritchard \& Davies, 2002; Schön, 1983), but nurses must find time and energy to practice reflection (Fowler, 2014). Researchers have described the benefits and advantages of using reflection and highlighted negative feelings associated with it. For example, Oluwatoyin (2015) stated:

The process can be manipulated to meet the expected outcomes of the practice; reflective practice may cause psychological stress; [nurses] usually reflect on negative issues; [and nurses become frustrated] ... not able to solve problems that were identified during reflection. (p. 31) 
Nursing educators and scholars understand the barriers and challenges of using self-reflective practices (Mann et al., 2009; Oluwatoyin, 2015). Mann et al. (2009) suggested, “Activities to promote reflection are now being incorporated into undergraduate, postgraduate, and continuing medical education, and across a variety of health professions" (p. 595). Nursing educators want nurses and students to use reflective practice because it ultimately leads to professional development and higher quality patient care (Mann et al., 2009; Oluwatoyin, 2015).

\section{A Summary of Self-Reflective Practice for Nursing Professionals}

Emotional intelligence and awareness of one's emotions are valuable skills for leadership development in nursing. Additionally, self-awareness is a skill required for nurses to develop self-reflection competence. Researchers, however, suggest nurses must have adequate time to effectively use self-reflective practices in the clinical setting (Edwards, 2003; Fowler, 2014). The lack of time for reflection is one of the main barriers to using self-reflective practices, along with minimal organizational support and the perception by some nurses using self-reflection lacks practical application. Despite these barriers, self-reflection is valuable to nursing educators (Mann et al., 2009; Oluwatoyin, 2015). Nursing students are being exposed to the value of using self-reflective practices during their clinical practicum. Nursing educators want students to use self-reflection because the goal is to value their "strengths and to develop different, more effective ways of acting in the future" (Oluwatoyin, 2015, p. 29). Nursing educators and mentors understand the benefits of teaching nursing students about reflective practices, especially in an effort to help them communicate feelings of stress during their clinical practice (Bailey \& Graham, 2007; Chong, 2009). 


\section{A Guide for Nursing Students to Use Reflective Practice}

Nursing educators seek to understand the perceptions of student nurses regarding their personal experiences using self-reflection (Chong, 2009). To understand if nursing students demonstrate similar feelings toward self-reflective practices as nurses do in the clinical setting, Chong (2009) developed a study to explore the perceptions of final-year nursing students on the usefulness of reflective practice.

Self-reflection is useful to nursing students (Chong, 2009). Nursing students report having a positive perception of self-reflective practices (Chong, 2009). Student nurses are specifically learning about self-reflective practices as they prepare to complete nursing education prior to entering the profession (Joyce-McCoach, Parrish, Andersen, \& Wall, 2013). Using selfreflective practices early in their careers may help nurses process their emotions effectively later. According to Chong (2009), "Practicing reflection, it would enhance their learning activity and motivate self-directed learning needs and foster lifelong learning" (p. 119). Nursing students in Chong's study reported having a positive perception of self-reflective practices; however, limited information was provided on how nursing students used reflection to process their feelings (Bailey \& Graham, 2007; Chong, 2009). More research is needed to understand how nursing students view their experiences using self-reflective practices to process emotions.

\section{Managing Emotions Triggered by Reflective Practice}

In this section, I provide a review of studies related to the main objective of this study: to reveal the experiences of nurses using self-reflective practices in the clinical setting. Rees (2013) developed a phenomenological study to understand final-year nursing students' experiences and how "engagement with reflective practices enabled the participants to manage the distressing emotional challenges and labour of nursing work" (p. 48). Rees (2013) purposefully recruited 
students who "felt that they had experienced the phenomenon of learning through reflective practices" (p. 49). Recruiting participants who want to use self-reflective practices aligns with Fowler (2014), who said nurses must want to find time for reflection. By recruiting students who wanted to engage in self-reflective practices, Rees (2013) better undersood why and how nursing students self-reflect. Each of the 10 nursing students participated in one audiotaped, in-depth interview; their interviews were transcribed verbatim. Phenomenological analysis of the 10 transcripts revealed two findings: (a) emotional distress, personal suffering, and the reflective prompt and (b) developing personal boundaries for professional engagement. All 10 participants in Rees' (2013) study

described the experience of learning through reflection prompted by their response to significant, often distressing, events. These types of affective triggers alerted the participants to their need to make sense of something that mattered to them on a personal level, and reflection helped the students to develop background resources and strategies to manage the emotional challenges inherent in caring work. (p. 49)

The reason participants reflected was a result of their need to empathize with their patients and to deal with their specific circumstances (Rees, 2013).

Emotional distress, personal suffering, and the reflective prompt. According to Rees (2013), participants were chosen based on their willingness to understand the affective nature of their personal feelings in response "to the personal stories and circumstances of those whom they were caring for" (p. 49). Through reflective practice, participants are able to better understand when and how to process their feelings effectively and to learn from each patient experience (Rees, 2013). For example, one participant who used reflective practice was better able to "hold herself open to others' unique situation" (Rees, 2013, p. 50). Thoughtful reflective practice 
challenged the personal values and beliefs of this participant and "enabled her to consider how she could be more receptive to, and understanding of, others' experiences and less judgmental in her care" (Rees, 2013, p. 50). Not all participants, however, effectively used reflection. One participant had trouble making "personal sense of another's experience" (Rees, 2013, p. 50). This participant displayed a "tactic" some nurses use "who do not want to suffer themselves whilst their patients are distressed" (Rees, 2013, p. 50). What reflection did for this participant was to help her understand professional boundaries and to "decide how she would allow herself to suffer with her patients" (Rees, 2013, p. 50).

When nurses want to understand their feelings, as opposed to hiding them or remaining stoic, patient care experiences can be triggers that inspire nurses to use reflective practices to connect with patients authentically (Boyd \& Fales, 1983; Rees, 2013). For participants in Rees' (2013) study, nurses wanted to understand more about their feelings of stress used self-reflection. Rees (2013) stated, "Reflection helped the students to develop background resources and strategies to manage the emotional challenges inherent in caring work" (p. 49). Interestingly, Rees (2013) suggested, "Reflective practices may contribute to a humanizing value framework for healthcare, which values the insiderness, agency, and uniqueness of both the practitioner and the fellow humans in their care" (p. 51). Rees' (2013) study "differentiated between authentic reflective practice" and "academically driven" reflective practices by seeking participants who wanted to learn "what nursing was for them, how it affected them, and how it embraced them as individuals" (pp. 48-49). Thus, participants who wanted to learn more about their emotional responses were the same students who wanted to engage in using self-reflective practice (Rees, 2013). 
Developing personal boundaries for professional growth. Reflective practice enabled one student to address her feelings authentically and to learn from her patient-care experience. According to Rees (2013):

Ellen described how awkward and embarrassed she felt when the father of a young patient who had just died immediately hugged her rather than one of his family.

Reflection on the experience enabled Ellen to feel at ease with the situation and more able to respond prospectively in a more human and embodied way. (p. 50)

Learning from experience occurs when participants reflect and understand the movement between their personal emotions and their professional behaviors (Rees, 2013). Rees (2013) stated, "Reflection enabled the participants to understand the individual nature of the emotional challenge of nursing and what it meant to them personally to be a nurse" (p. 50) and used the process of reflective practice "to try to locate themselves in the context of care" (p. 50). Students used reflective practice to learn how to provide "authentic and embodied care" (Rees, 2013, p. 50). Reflecting and addressing emotions can help nurses in two ways: (a) in providing patientclinician-centered care and (b) in developing in their professional roles by learning from their unique experiences.

\section{Care for Critical Patients and Reflective practices}

Although Rees (2013) described why nursing students want to use self-reflective practices, little is known about how these nursing students use reflective practices or the types of practices they use to manage emotions successfully. Rees (2013) indicated patient interactions and distressing emotions may trigger nursing students to use reflective practice. We also know nursing students use reflection to develop "personal boundaries for professional engagement" (Rees, 2013, p. 50). What remains unclear is how experienced nurses use reflective practices 
when they provide care to critical patients. Researchers must focus on experienced nurses who provide care in critical environments like emergency rooms or cancer units because they are more likely to experience continuous feelings of distress and burnout than are less experienced nurses (Altounji et al., 2012; Barnard et al., 2006; Boyle \& Bush, 2018; Enskär, 2012; Zander et al., 2010).

Limited information exists on the experiences of nurses in critical environments and their experiences using self-reflective practices. Therefore, researchers learn about reflective practices from the point of view of nursing mentors. For example, Gelabert-Vilella et al. (2014) explored stress factors among mental health nursing students. Although self-reflective practices were not the main purpose of this study, Gelabert-Vilella et al. (2014) described how "mentor nurses ... emphasized the importance of reflective practice and students' self-management of their learning experiences" (p. 79). Nursing educators understand the value of learning from experience and express the importance for mental health nursing students to use self-reflection. King-Okoye and Arber (2014) focused on nursing students who provide direct care to cancer patients and experience emotional distress in the process.

\section{Reflective practice and students caring for cancer patients. King-Okoye and Arber} (2014) developed a phenomenological study to explore the experiences of second- and third-year nursing students who provide care to cancer patients in the United Kingdom. Focus group interviews were conducted with 20 student nurses. Five themes emerged from the focus group interviews: (a) communication with patients, (b) impact on self, (c) I feel lost, (d) we should be prepared, and (e) cancer and end-of-life. According to King-Okoye and Arber (2014), student nurses responsible for providing care to cancer patients need to feel support, which should include "psychosocial care and specific skills in communication and reflective practice such as 
breaking bad news, handling strong emotions, and providing supportive care" (p. 448). Teaching nursing students to use reflective practices can help them "feel supported and develop confidence in their capacity for care for people with cancer" (King-Okoye \& Arber, 2014, p. 441).

Furthermore, it may be important for nurses to develop self-reflective practices at the beginning of their careers because the skill is rapidly becoming part of the overall requirements in clinical practice (Oluwatoyin, 2015).

Students reflecting on their clinical practice. Similar to Rees' (2013) study, Karimi, Haghani, Yamani, and Kalyani (2017) explored the perceptions of Iranian nursing students on the consequences of self-reflection in clinical practice. Specifically, Karimi et al. (2017) focused on the "process of reflection at two nursing schools" (p. 5192). Data were collected using indepth, semi-structured interviews with a purposive sampling of 20 students who had "at least one semester practical training" (Karimi et al., 2017, p. 5192) and were "interested in participating and recounting experiences in the study" (p. 5192). Open-ended interview questions were focused on "the experience of reflection in a clinical setting" (Karimi et al., 2017, p. 5162). Examples from Karimi et al.'s (2017) protocol included, “Tell me about one of your clinical experiences that you had to think and reflect more. What was the consequences of this reflection on your clinical experience?" (p. 5193) and "Who helped you achieve these consequences?" (p. 5193). Analysis of the interview transcripts data reveled two main themes: (a) movement toward professionalism and (b) self-actualization of emotions. Subcategories for the theme movement toward professionalism were function modification, sharing experiences, and generalizing experiences. The subcategories for the second theme, self-actualization of emotions, were inner satisfaction and peace of mind. 
Karimi et al.'s (2017) suggested reflection is a process with personal and professional characteristics that motivate nursing students to learn how to provide higher quality care to their patients. Rees (2013) also reported similar findings where nursing students who used reflective practice processed personal feelings and learned from the experiences in the clinical setting. According to Karimi et al. (2017), "At the personal level, reflection leads to positive feelings such as satisfaction, happiness, and self-confidence" (p. 5195). Students reflected on their experiences and were willing to express their emotions, an exercise that "was the growing factor and catalyst for reflection" (Karimi et al., 2017, p. 5195). These findings are similar to Rees' (2013) study: (a) nursing students had a positive experience using reflective practice and (b) nursing students were triggered to use self-reflection to learn more about their feelings. Karimi et al. (2017) also described the professional level where "reflective students place the patient at the center of care" (p. 5195) and "use reflection on their experiences to provide accurate, proper, and quality caring services" (p. 5195). Rees (2013) and Karimi et al. (2017) provided evidence that nursing students who provide care to cancer patients or work in the clinical environment can benefit from using self-reflective practices.

\section{A Summary of Nursing Students and Their Perceptions Using Self-Reflection}

Nursing educators believe teaching nursing students about self-reflective practices is beneficial (King-Okoye \& Arbor, 2014). Nursing students use self-reflective practices to communicate and process their emotions in the clinical setting, and it may translate into better care for the patients (Bailey \& Graham, 2007; Chong, 2009; Rees, 2013). Reflective practice is mostly used to help students learn how to manage their feelings of stress (Gelabert-Vilella et al., 2014; Karimi et al., 2017; King-Okoye \& Arber, 2014). The critical care setting can be emotionally challenging for nursing students. Learning to process emotions and using self- 
reflective practices can encourage nursing students to learn and develop confidence in their clinical skills. This confidence is especially evident with nursing students who care for cancer and mental health patients (Gelabert-Vilella et al., 2014; King-Okoye \& Arber, 2014).

Nursing students who described their experiences using self-reflection in the clinical setting described how they effectively processed their emotions and experiences to develop both personally and professionally (Karimi et al., 2017). There is some evidence to suggest nursing professionals use self-reflective practices in a similar manner as students do to process emotions in an effective manner and provide patients with higher quality care (Gustafsson \& Fagerberg, 2004). Therefore, the next section is a discussion of what nursing professionals use selfreflection for and what they reflect about.

\section{Nurses and the Ramifications of Using Self-Reflective Practices}

Self-reflection is an ongoing process and may improve with time and experience (Edwards, 2003). Gustafsson and Fagerberg (2004) set out to understand this improvement and the "implications of nurse's reflections, what do they reflect about, and how do they deal with their reflections?” (p. 271). Reflection was described as integral to developing as a registered nurse (RN). The aim of Gustafsson and Fagerberg's (2004) study was

to describe RNs' experiences of reflection in relation to nursing care situations, to understand how RNs use reflection in their daily work: What are the implications of the actual nursing care situations that RNs' reflect upon? What do the RNs feel were the consequences of reflection in nursing care situations, in relation to their professional development? (p. 273)

According to Gustafsson and Fagerberg (2004), RNs reflect on nursing experiences by considering past situations and by using clinical nursing supervision. The content of RNs' 
reflections include ethical considerations, courage, imagination, and consideration of the consequences of reflective nursing care that recognizes and empathizes with the unique person. Gustafsson and Fagerberg (2004) stated:

To be open and rely on one's feelings and one's imagination implies that the nurse is mature. The nurses use their feelings according to what can and should be done and they can train themselves to move beyond certain restrictions" (p. 276).

As in Rees' (2013) study, participants in Gustafsson and Fagerberg's (2004) study reported using self-reflective practices to manage their feelings in a creative and constructive manner. Gustafsson and Fagerberg provided information on the experience of RNs' using self-reflective practices, but researchers do not agree on the types of reflective practice that helps nurses improve patient care.

\section{Reflective Practice Intervention}

A debate about "the benefits of and problems with the use of reflective practice" (PedenMcAlpine et al., 2005, p. 449) inspired the researchers to discuss the "design, evaluation and outcomes of a reflective practice intervention (RPI) that taught pediatric critical care nurses how to incorporate a family intervention into their practice" (p. 449). Using a phenomenological research approach, eight nurses from two children's hospitals in the United States participated in the study. Individual interviews were conducted with participants 3 to 4 weeks after the RPI to address any changes in their care (Peden-McAlpine et al., 2005). Interviews were audio recorded and transcribed verbatim. The design of the RPI was developed using "three educational strategies of narrative, role modeling, and reflective practice" (Peden-McAlpine et al., 2005, p. 496) to "transfer knowledge from the expert family Clinical Nurse Specialist (CNS)" (p. 497) to novice nurses. 
According to Peden-McAlpine et al. (2005), the narrative "involved a situation where the expert family nurse was supporting a family emotionally and coaching them with decisionmaking about a severely brain damaged child ... to produce positive benefits for the family" ( $\mathrm{p}$. 497). Second, a CNS was used to role model. Third, the RPI was a reflective discussion between the expert nurse and the more novice nurse. Prior to conducting interviews, participants received instructions via mail that asked them to "reflect on their experiences of participating in the RPI and to recall specific 'stories' of their practice with families during and after the intervention" (Peden-McAlpine et al., 2005, p. 497). At the completion of the interviews, participants were asked two specific questions on the RPI: "Can you recollect anything about family care that you think affects your practice today?" and "How is your practice with families different now than before you participated in this study?" (Peden-McAlpine et al., 2005, p. 497). Interview transcripts were analyzed using van Manen's (1990) phenomenological approach.

Peden-McAlpine et al. (2005) demonstrated how reflective practices can be used to help nurses bridge the gap between theory and clinical practice. Furthermore, the nurses in the study improved their care by "reflecting on and questioning prior assumptions, redirecting their thinking and individualizing care based on the unique needs of the family" (Peden-McAlpine et al., 2005, p. 500). Pediatric critical care nurses who use the RPI in the clinical setting can improve the quality of care they provide to patients and their families. Nurses who work with critical patients or who work in emergency departments seem to benefit from using reflective practices (Gelabert-Vilella et al., 2014; King-Okoye \& Arber, 2014; O’Neill, Johnson, \& Mandela, 2019). 


\section{Reflective Practice Groups}

Reflective practice groups are useful for managing feelings of burnout in liaison psychiatry nurses working in emergency departments (O’Neill et al., 2019). O'Neill et al. (2019) explored the experiences of nurses attending a facilitated reflective practice group over several months (e.g., 9 months). Four themes emerged from the reflective practice group: (a) sharing and learning, (b) grounding and perspective, (c) space, and (d) relationships. O'Neill et al. (2019) stated reflective practice groups "may offer benefits to help mitigate the impact of and reduce occupational stress and burnout" (p. 91). This research indicated reflective practice groups are useful for liaison psychiatry nurses working in emergency departments (O’Neill et al., 2019).

\section{Summary and Key Findings of Reflective Practice in Nursing}

Self-reflective practices have the capacity to change behaviors in a way that leads to realizing new perspectives (O’Neill et al., 2019). Additionally, nurses who reflect use imagination and learn creative ways to develop insight and expertise in their professional roles (Gustafsson \& Fagerberg, 2004; Walker, 2008). Walker (2008) stated:

Nurses do not consider themselves to be expert in the areas in which they work ... structured reflection can assist nurses to identify areas of practice in which they operate in an expert manner ... to value their work as expert professionals. (p. 5)

What remains unclear are the perceived results of using self-reflection by pediatric oncology nurses with 10 or more years of bedside experience. My review of the literature did not yield any articles that specifically address pediatric oncology nurses and learning outcomes that occur as a result of their use of self-reflection. Studies that did cover self-reflective practices and pediatric oncology nurses were few. I discuss and critique these studies in the next section. 


\section{Review of the Literature on Pediatric Oncology Nursing}

Four studies that directly or indirectly include pediatric oncology nurses and selfreflective practice are used in this review of the literature. These four studies also form the foundation for developing this phenomenological study and are discussed in chronological order starting with Enskär (2012) and followed by Charalambous and Kaite (2013), Hines and Gaughan (2014), and Mirlashari et al. (2017). The next section is a brief introduction to all four studies.

Enskär's (2012) study is titled, "Being an Expert Nurse in Pediatric Oncology Care: Nurses' Descriptions in Narratives." This study was developed so researchers could address the insufficient training of specialist nurses in Sweden. Enskär (2012) looked at different roles that meet the needs of expert-level nurses such as CNSs and advanced practitioner nurses (APNs). The CNS and APN roles are not well established in Sweden. Therefore, Enskär (2012) developed a study to understand the perceptions of "newly educated nurses in pediatric oncology regarding the role of an expert nurse in pediatric oncology" (p. 152).

Charalambous and Kaite's (2013) study is titled "Undergraduate Nursing Students Caring for Cancer Patients: Hermeneutic Phenomenological Insights of Their Experiences.” According to Charalambous and Kaite (2013), more cancer patients pass away while under the care of nurses as opposed to under the care of doctors in Cyprus. Nursing professionals are directly involved in "caring for patients who are dying or have a terminal illness and are faced with the process of dying. Working with these patients and their families can be emotionally demanding and challenging" (Charalambous \& Kaite, 2013, p. 2). Although Charalambous and Kaite (2013) did not directly address pediatric oncology, they did explore experiences of undergraduate nursing students who have prior experiences in pediatric oncology departments. Therefore, 
Charalambous and Kaite (2013) aimed to "explore the experiences of the undergraduate students when they come face-to-face with cancer care during their clinical placements. The main objective being to help nursing students understand and interpret their experiences" (p. 3).

In the United States, Hines and Gaughan's (2014) study, "Pediatric Nurses Acknowledging Praxis: Recognizing Caring in Reflective Narratives," was devised to illuminate the need to explore the concept of caring among pediatric nursing professionals. Caring is not simply a performance aimed at managing medical tasks and providing care to patients and families (Hines \& Gaughan, 2014). Caring is a form of praxis Hines and Gaughan (2014) described as a "synthesis of thoughtful reflection, caring, and action within theory and researchdriven practice. Caring is the foundation that provides a framework for nursing praxis. Praxis transforms practice through reflection" (p. 26). Caring is a practice that connects the patient and nurse through a process of self-transformation. For this reason, Hines and Gaughan (2014) explored the experiences of pediatric nurses using reflective narratives. The aim of their study was to "explore caring within pediatric nursing practice, specifically how reflective narrative can be used to capture the essence of caring" (p. 27).

Like Charalambous and Kaite (2013), Mirlashari et al. (2017) explored the experiences of undergraduate nursing students during their first clinical rotations in pediatric oncology in Iran with their study titled, "The Experiences of Undergraduate Nursing Students and Self-Reflective Accounts of First Clinical Rotation in Pediatric Oncology." According to Mirlashari et al. (2017), "Preparing undergraduate nursing students to be safe, knowledgeable, and critical selfreflective practitioners is an acknowledged aim of most undergraduate nursing programs worldwide" (p. 22). The nature of the pediatric oncology setting and the demands of providing care to critical patients can be challenging for students during their clinical rotations. Pediatric 
oncology nurses can mitigate the effects of stress and burnout by using reflective practices, such as reflective journaling. Mirlashari et al. (2017) stated, "Reflection is a crucial element in a clinical learning environment ... Keeping a reflective journal is thought to help students to critically self-reflect upon their clinical and life experiences and to increase student awareness of their behavior and care decisions" (p. 23). Mirlashari et al. (2017) explored "student perceptions and self-reflective journal accounts of what it was like to care for children with cancer and their family throughout the course of their first practicum on a pediatric oncology unit" (p. 23). Their goal with the study was to support the growth and development of student nurses, better prepare them for the emotional demands of pediatric palliative care, and help clinical instructors understand the unique experiences of undergraduate students (Mirlashari et al., 2017).

According to Enskär (2012), Charalambous and Kaite (2013), Hines and Gaughan (2014), and Mirlashari et al. (2017), exploring the experiences of nursing professionals and students regarding self-reflective practice is not only crucial for understanding perceptions of end-of-life care, it is part of the professional development of nurses to become expert-level clinicians. Expert-level nurses who use self-reflective practices to explore their emotions in a healthful manner and continue learning and caring for critical patients are valuable to the field of pediatric oncology.

\section{Methods Researchers Used to Explore Self-Reflective Practices}

All four studies described in the previous section used different methodologies to explore the perceptions of nursing professionals and students. Enskär (2012) used a qualitative design with participants' narratives as the main source of data. Reflective stories are an ideal method to understand how participants make sense of their unique experiences (Enskär, 2012). Enskär (2012) reported, "The narrators do not merely communicate a simple story to the reader, they 
also clarify and reflect on the past, justify their past behaviors, and link the past to their present thinking and actions" (p. 152). In 2003, a 2-year course on pediatric oncology nursing care was developed in Sweden and "[three] groups, totaling 68 nurses, have graduated as of 2010" (Enskär, 2012, p. 152). Along with the final exam and end-of-year evaluation, 68 nurses were asked to write their narrative accounts about being expert nurses in pediatric oncology.

Out of 68 nurses, 66 responses were collected and analyzed in Enskär's (2012) study. Of the 66 participants, two males and 64 females were between the ages of 24 and 57 with $42 \%$ of the group between the ages of 35 and 44. Most participants had between 11 and 20 years of nursing experience. According to Enskär (2012), “The nurses' experience in pediatric oncology was diverse, with about one third working less than 6 years and 10 years, and one third more than 10 years" (p. 153). All 66 participants shared unique experiences working with pediatric patients. Many of the nurses $(n=42)$ had direct pediatric oncology experience, while other nurses $(n=23)$ had general pediatric care experience. Participants received written instructions on how to write their narratives, and data were collected 2 months before final exams in 2005 , 2007, and 2010. Approximately 4 weeks after the written instructions were sent to each participant, narratives were completed and returned via email or letter, and "the narratives were of various lengths, ranging from two to eight pages, with the majority being three to four pages" (Enskär, 2012, p. 153). Content analysis was performed on the written narratives, as suggested by Krippendorf (2004), and a working model, based on Graneheim and Lundman (2004), was used to generate categories from the abstraction of text (Enskär, 2012).

Charalambous and Kaite (2013) used a hermeneutic phenomenological design, using Ricoeur's approach “to uncover the significance of experienced feelings and thoughts behind students' stories ... to help them understand and interpret the situation of each patient" (p. 3). 
This phenomenological approach of interpreting text included three stages: (a) naïve reading, (b) structural analysis, and (c) comprehensive understanding (Charalambous \& Kaite, 2013). Data were collected using reflective (narrative) diaries of 12 undergraduate nursing students, and each was used for interpretation. These students were experiencing clinical practice for the first time in oncology, hematology, and pediatric oncology departments, and hospice settings were selected "based on their willingness to take part in the study" (Charalambous \& Kaite, 2013, p. 3). Reflective diaries were not structured in any way to elicit meaningful experiences to resurface.

Participants had a 2-hour session introducing reflective diaries and practiced using clinical scenarios to help them feel competent in their reflective writing (Charalambous \& Kaite, 2013). Although specific questions were not used to guide participants in their reflective diaries, themes such as "feelings, perceptions, concerns, fears, and experiences of their first encounters with the clinical setting and the care itself" (Charalambous \& Kaite, 2013, p. 4) were used to help guide their writing. According to Charalambous and Kaite (2013), the "decision to implement a written narrative approach as the means to explore the students' experiences lies on the fact that this approach is a text-oriented interpretation, and inasmuch as text are, among other things, instances of written language" (p. 4). Each participant was given the opportunity to record their experiences during their clinical practice experiences, two times a week for a total of 6 weeks (Charalambous \& Kaite, 2013). Using reflective diaries and an unstructured approach to writing provided students with the opportunity to record and "recall the event at an ease of time, which would allow a more accurate passage of the experience on text" thoughtfully and in detail (Charalambous \& Kaite, 2013, p. 4).

In Hines and Gaughan's (2014) study, a total of nine participants (i.e., pediatric surgery nurse, pediatric rehabilitation specialist, pediatric case manager, holistic CNS, and family nurse 
practitioner) fit the inclusion criteria and were recruited to participate. Hines and Gaughan (2014) stated, "The researchers sought pediatric nurses actively practicing to write narratives exemplifying caring within practices" (p. 27). None of the nurses had direct pediatric oncology experiences. Participants' experience ranged between 2 to 37 years. Hines and Gaughan (2014) used a data collection process with four distinctive steps:

1. "Asking nurses to reflect on a time in their practice that captured the essence of caring.

2. "Asking nurses to write a narrative capturing details of that time.

3. 'Asking nurses to use Johns' (2009) model for structured reflection (MSR) to analyze their narrative.

4. “Researchers analyzing narratives for common themes using van Manen's (1990) layers to phenomenological analysis." (p. 27)

Johns' (2009) MSR was used by each participant to analyze their own reflective narratives. Participants were provided a structured approach considering that none of them had been exposed to reflective narrative prior to the start of this study (Hines \& Gaughan, 2014). According to Hines and Gaughan (2014), Johns' (2009) MSR consisted of 17 statements:

1. "Focus on a description of an experience that seems significant in some way.

2. "What issues are significant to pay attention to?

3. "How do I interpret the way people are feeling and why they felt that way?

4. "How was I feeling and what made me feel that way?

5. "What was I trying to achieve and did I respond effectively?

6. "What were the consequences of my actions on the patient, others, myself? 
7. "What factors influence the way I was/am feeling, thinking, and responding to the situation?

8. "What knowledge did or might have informed me?

9. "To what extent did I act for the best and in tune with my values?

10. "How does the situation connect with previous experiences?

11. "How might I reframe the situation and respond more effectively given this situation again?

12. "What would be the consequences of alternative actions for the patient, others, and myself?

13. "What factors might constrain me responding in a new ways?

14. "How do I now feel about this experience?

15. “Am I more able to support myself and others better as a consequence?

16. "What insights have I gained?

17. “Am I more able to realize desirable practice?” (p. 28)

All participants were given 4 to 6 weeks to write and analyze their reflective narratives (Hines \& Gaughan, 2014). Upon receiving the reflective narratives, Hines and Gaughan (2014) conducted data analysis for themes and meanings “using van Manen’s layers of hermeneuticphenomenological analysis" (p. 28).

Mirlashari et al. (2017) explored the perceptions of 25 Iranian undergraduate students (19 women and six men) in their final year of their nursing program and used "conventional content analysis of data from student weekly face-to-face semistructured interviews and daily selfreflective journaling” (p. 23). Participants' ages ranged from 21 to 24 years. Mirlashari et al. (2017) stated, "None had been previously been assigned a clinical rotation in pediatric oncology 
and none had experience in self-reflective journaling" (p. 23). Data collection began at the start of the students' 2-week clinical rotations and ended after the rotations were completed (Mirlashari et al., 2017). All students received information on self-reflective journaling and were introduced to Gibbs' (1988) reflective cycle.

Mirlashari et al. (2017) provided each participant with instruction "on the stages of the cycle, including description, feeling, evaluation, conclusion, and action" (p. 24). The data collection procedures involved both interviews and self-reflective journals. Two 30-minute, faceto-face interviews were conducted (audio recorded), and students were asked to write selfreflective journals that pertained to these prompts:

Tell me about your thoughts and experiences of taking care of children with cancer. What is it like for you, to be on oncology pediatric unit and to be involved in providing care to the children you were assigned and their family? You are now in your second week of your practicum, what is like for you to be in involved in providing care to the children you were assigned and their family? (Mirlashari et al., 2017, p. 24)

These prompts were also addressed during the face-to-face interviews. Data analysis involved conventional content analysis, which combined interview transcripts and participants' selfreflective journals, as described by Hsieh and Shannon (2005). The research team conducted data analysis until "consensus was achieved on the codes and categories" (Mirlashari et al., 2017, p. 24).

All four studies used a qualitative methodology to explore the perceptions of undergraduate students and nursing professionals who share similar experiences in the pediatric clinical setting. Methods of collecting data were reflective narratives, diaries, journals, and faceto-face interviews. Researchers in each study had a unique method of analyzing its data, which 
ranged from content analysis to interpretive phenomenological analysis where text was the main source of data. Students were exposed to reflective journaling during their final year of nursing school; nursing professionals were asked to write reflective narratives or diaries. Students who used self-reflective journals were also interviewed using similar guidelines. Only one research team combined both journals and interview transcripts during its data analysis phase (Mirlashari et al., 2017). Most importantly, questions asked in each study focused on understanding participants' emotions about the nature of the pediatric oncology setting and about professional development in clinical practice.

\section{A Need to Continue Exploring Self-Reflective Practices}

Distinct methods of exploring the perceptions of participants were demonstrated in each study, yet they share similar result patterns. Using narrative accounts to explore participants' perceptions of an expert nurse, Enskär (2012) concluded reflection is regarded as a valuable characteristic of expert-level nurses. Participants in Enskär's (2012) study also stated, to reflect authentically and with deep insight, nurses must be given time to assimilate theory and clinical practice or else levels of expertise cannot be achieved. Using reflective diaries to explore the perceptions of undergraduate students during their first clinical placements in cancer care, Charalambous and Kaite (2013) stated "being self-reflective" (p. 6) was described as the students' capacity to evaluate their "own abilities, behaviors, and reactions towards the patients" (p. 6). Being self-reflective is not only experienced in a positive manner, but is also explained in negative terms (Charalambous \& Kaite, 2013).

Like Charalambous and Kaite (2013), participants in Hines and Gaughan's (2014) study used reflective narratives to capture the essence of care in the pediatric clinical setting. Hines and Gaughan (2014) revealed, by using reflective narratives, participants illuminated the 
interconnected world often neglected or hidden in the clinical practice setting. According to Hines and Gaughan (2014), the use of reflective narratives allowed participants to "understand and appreciate the complexities and meanings of the human experience of caring" (p. 33).

Using face-to-face interviews and self-reflective journals to explore undergraduate students' perceptions during their first clinical rotations in pediatric oncology, Mirlashari et al. (2017) discovered results similar to Charalambous and Kaite's (2013). Mirlashari et al. (2017) revealed reflective journals expose the experiences of nursing students who come face-to-face with pediatric cancer patients. Emotions are a mixture of shock and terror during the first interactions with critical patients. These feelings of terror and shock transform or melt away into meaningful experiences as students spent more time on the unit. When students were asked about their feelings on using self-reflective journals, "most reported that they found it useful and believed self-reflective journaling gave them access to deeper layers of understanding and that it had a positive impact on their emotion and nursing practice" (Mirlashari et al., 2017, p. 26). Students in this study used self-reflective journaling and, as a consequence, learned how to be mindful when providing care to patients and their families in the future (Mirlashari et al., 2017).

While self-reflective practices in the context of pediatric oncology nursing were used in the previous studies, none of the researchers specifically focused on experienced pediatric oncology nurses who use self-reflective practices in the clinical setting. Furthermore, no acknowledgement of nurses who willingly choose to use self-reflection in their daily lives (as opposed to nurses who do not) was noted. As previously stated, there is a difference in the effectiveness of using self-reflection when nurses want to engage in the process authentically (Fowler, 2014; Karimi et al., 2017; Rees, 2013). All four research teams in this review addressed 
reflection as an essential aspect of nursing practice and professional development. Being reflective, however, does not always imply uplifting experiences.

Researchers have shown using reflective practices can be positive, but nursing professionals and students mainly reflect on negative situations (Charalambous \& Kaite, 2013; Mann et al., 2009; Mirlashari et al., 2017; Oluwatoyin, 2015). Mirlashari et al. (2017) addressed this issue of negativity by stating nursing students need more time to experience the pediatric oncology setting for their reflective experiences to transform into meaningful practices. None of the authors, however, address reflective practices during the care process, but such practices are implied by their choice of methods: reflective narrative, diaries, or journals. Also not addressed is the issue of nursing professionals' lack of time for reflection during clinical hours. Instead, a structure or format of reflection is accomplished away from the clinical setting or during downtime when nurses and students have personal time to engage in deep reflection.

\section{Limitations and Critiques}

Each of these studies contains information pertaining to reflective practices and the implications of using such practices to develop as nursing professionals. The studies also have distinct limitations that must be addressed. Enskär (2012) used narrative methodology, a form of hermeneutic phenomenology where the data are analyzed using participants' written words. A criticism of the study is participants in a 2-year course for nurses in pediatric oncology care are asked to write their narratives 2 months prior to their final exam and evaluation. Although authentic narratives may have been collected, the amount of effort given to the narratives ranged from two to eight pages (average three to four pages) in the span of 4 weeks. The author described only the amount of paper used to write the narratives without describing the detail and depth of participants' narratives. Another limitation of Enskär's (2012) study is 'it was not 
possible to compare the narratives of the more experienced nurses with those of the less experienced ones" (p. 158). This comparative information is necessary considering selfreflection can improve over time, making comparing nurses with varying levels of clinical experience difficult (Mann et al., 2009). Enskär (2012) highlighted the need to explore the experiences of pediatric oncology nurses who have an authentic understanding of self-reflective practices. Comparing the self-reflective practices of pediatric oncology nurses to those of less experienced nurses could provide valuable information helpful to novice nurses experiencing similar situations and feelings (Oluwatoyin, 2015).

Reflective narrative is important to understand the experiences of nurses but not necessarily to understand the process of self-reflection. In Charalambous and Kaite's (2013) study, the rationale for using reflective narratives is to use a method of collecting data (interpretive phenomenology) that was useful to describe the experiences of students in the clinical setting. Enskär (2012) used a similar methodology to interpret the narratives of students. Charalambous and Kaite (2013) stated, "It is the authors' belief that data regarding the experiences of the students should also be collected through written narratives (diaries), a means that allows the narrator to describe the experience in more detail without the pressure of the interview" (p. 2). The problem is instead of using reflective practices as a process of selfawareness, understanding, and changing past behaviors (Chacko \& Sreerenjini, 2012; Pool, 2018), Charalambous and Kaite (2013) used reflective narratives as a method for researching lived experiences rather than as a method for teaching students about being reflective.

The aim of reflective practice is "to develop professional actions that are aligned with personal beliefs and values" (Oluwatoyin, 2015, p. 28). From the students' narratives, it is not apparent reflection was performed. Rather, students described their feelings and thoughts without 
expressing if they were learning or how they were beginning to critically examine their behaviors (Oluwatoyin, 2015). According to Charalambous and Kaite (2013), students recorded their experiences during their clinical practice, twice a week and within a period of 6 weeks . . the reason for choosing to use the diaries was the opportunity provided to students to recall the event at an ease of time. (p. 4)

It appears students were merely recalling their experiences and not reflecting on their experiences.

An experience can trigger students to use reflective practices (Boyd \& Fales, 1983). This is not the case, however, in Charalambous and Kaite's (2013) study. Students were asked to write their reflective narratives at their earliest convenience. This was also evident in Enskär's (2012) study where students were asked to write their narratives along with their coursework. According to the nursing literature, reflective practices are part of the nature of being a nurse; however, the process can be manipulated to meet the expected outcomes of the practice (Oluwatoyin, 2015). In Enskär's (2012) and Charalambous and Kaite's (2013) studies, students used reflective narratives to fulfill a requirement and not as a process of learning or selftransformation (Mann et al., 2009; Oluwatoyin, 2015).

Hines and Gaughan's (2014) study is different from Enskär's (2012) and Charalambous and Kaite's (2013) studies because it does not specifically address pediatric oncology nurses. The reason for including Hines and Gaughan's (2014) study in this review is it uses reflective narrative to illicit a deeper understanding of caring among pediatric nurses. A criticism of this study is that researchers seemed to assume that participants were skilled enough at demonstrating the process of reflection to analyze their own reflective narratives using Johns' (2009) MSR. Although seven out of nine participants in this study were nurses who had 12 or more years of 
clinical experience, they were not previously identified as being reflective in their everyday lives.

Similar to Enskär (2012) and Charalambous and Kaite (2013), Mirlashari et al. (2017) used self-reflective journaling as a method to better understand the experiences of students and not as a tool to enhance their reflective capacity (Mann et al., 2009; Oluwatoyin, 2015).

Mirlashari et al. (2017)

employed conventional content analysis of data from student weekly face-to-face semi structured interviews and daily self-reflective journaling ... [this] has the distinct advantage of helping researchers to gain direct information from the study participants without imposing preconceived categories. (p. 23)

Mirlashari et al. (2017) used self-reflective journaling, interview transcripts, and interpretive phenomenology to better understand the clinical experiences of students during their 2-week clinical rotations in a pediatric oncology unit. According to Mirlashari et al. (2017), prior to beginning the study, students were taught about reflective practices using Gibbs' (1988) reflective cycle. As previously stated, Gibbs' (1988) reflective cycle has six distinct stages: (a) description of the event, (b) feelings, (c) evaluation, (d) analysis, (e) conclusion, and (f) action plan. Although students did perceive reflective journaling to be useful, journaling along does not exemplify the nature of Gibbs' (1988) reflective cycle.

If the students ended up identifying actions plans, what actions were the students taking to address better patient care in the future? For example, Participant 4 said:

When I was writing the journaling I was thinking about the better ways of care we could provide as nurses for the family and child. In the unit the time is short and we do not have enough time to think about our behaviors. (Mirlashari et al., 2017, p. 26) 
Although Participant 4 was addressing ways that nursing practice could possibly change, there is little to no time in the clinical setting to truly reflect about their behaviors.

\section{Summary}

Reflective practices are essential to nurses (Mann et al., 2009). Self-reflection is not simply remembering and analyzing events, it is an authentic process where participants are inspired and experience a transformation of the mind, which generates change in the future (Oluwatoyin, 2015). Although reflecting on the past can be challenging, positive experiences using self-reflective practices have been reported despite the difficulty of reflecting on negative experiences. Studies have shown pediatric oncology nurses work in highly stressful environments and rarely have adequate time to spend on self-reflection (Hecktman, 2012; Mirlashari et al., 2017). Experienced pediatric oncology nurses who use self-reflective practices are valuable sources of information on practical ways to self-reflect while on duty. Nursing scholars have shown that nurses who identify with self-reflection are committed to achieving personal goals, able to describe their experiences, and able to relate with patients to gain new perspective and insights (Mann et al., 2009). A review of the literature pertaining to pediatric oncology nurses and nursing students, however, has revealed scholars are using reflective narratives to explore lived experiences but not to explore the need to teach nurses how to use beneficial self-reflective practices (Charalambous \& Kaite, 2013; Enskär, 2012; Hines \& Gaughan, 2014; Mirlashari et al., 2017).

\section{Addressing the Gap in the Literature}

According to Dolphin (2013):

Reflective practice is crucial to acknowledge that objectivity is impossible without first understanding that practitioners will have an effect on patient care ... via their body 
language and other non-verbal and verbal communication, and their thoughts and emotions. (p. 20)

For nurses, critical thinking and the ability to reflect on past experiences have a combinatory effect by helping them decide appropriate medical interventions during stressful events (Robert \& Petersen, 2013). I attempt to address the gap in the literature on experienced pediatric oncology lived experiences using self-reflective practices in the clinical setting. Nurses who have 10 or more years of bedside experience and who use self-reflective practices were asked questions that represented key areas from the existing literature: reflect on medical tasks, patient care as an art, and nurses' emotional and physical wellbeing. In this study, experienced pediatric oncology nurses were asked to reflect on their experiences and to describe the meaning of selfreflective practices in the clinical setting. Researches in the studies that were previously mentioned asked pediatric oncology nurses and nursing students to reflect on an event (i.e., the what of their experience) rather than to explore the experience of using self-reflection (i.e., the experience of the what). I aim to gather data from nurses to explore what the experiences of using self-reflective practices are and how they are used in the clinical setting.

\section{Conclusion}

I developed this study to address the gap in the literature by recruiting nurses who use self-reflection as an integral part of their clinical practice. This was accomplished by sending a detailed recruitment email to potential participants indicating the purpose of this study and the need to recruit nurses who make time for self-reflection. Although previous researchers have highlighted the experiences and perceptions of pediatric oncology nurses and nursing students caring for patients through narrative accounts, reflective diaries, and personal journals, evidence is lacking on the individual experiences of using self-reflective practices as described by 
participants who identify as being self-reflective. Developing qualitative research pertaining to nurses who make time for reflection is aimed at helping nursing professionals and educators. Nurses may develop an understanding of the processes of thinking and behaving that contribute to self-care. Additionally, integrating these practices into clinical routines could provide benefits to nurses who are suffering from burnout and could support nurses' self-care practices. 


\section{CHAPTER THREE - METHODOLOGY}

Transcendental (descriptive) phenomenology (TP) is a type of qualitative inquiry that focuses on understanding the phenomenon of human thoughts, emotions, values (ethics and morals), and perceptions (Moustakas, 1994). It is transcendental because it goes beyond the normal, everyday understandings of particular human phenomena (Moustakas, 1994).

Descriptive phenomenological research is useful to explore the direct experiences of participants experiencing particular phenomena (Creswell, 2014; van Manen, 2014). I developed this study to fill the gap in the nursing literature on the self-reflective practice experiences of pediatric oncology nurses with 10 or more years of experience. The guiding research question was: What is the lived experience of expert-level pediatric oncology nurses using self-reflective practices in patient care? I used a phenomenological interview process to ask participants to recall experiences in which they used self-reflection. The aim was to obtain full descriptions of human behavior and to determine what the experiences meant for the nurse participants. Participants recruited for this study were employed in a pediatric hematology/oncology unit at a metropolitan teaching hospital in Southern California.

Chapter 3 includes discussion on (a) phenomenological and natural attitudes (Christensen, Welch, \& Barr, 2017; Husserl, 1931), including the researcher's subjective statements and the imaginative variations, (b) the methodology of choice, (c) methods for using TP, (d) research ethics, (e) the rigor and trustworthiness of the study, and (f) the research methods and design. In phenomenological research, articulating the phenomenological attitude is crucial. Thus, Chapter 3 includes information that pertains specifically to me as the primary researcher. My voice as the primary researcher throughout Chapter 3 helps reveal my natural attitude, as distinct from the phenomenological attitude, along with my researcher's subjectivity 
statement. The natural and phenomenological attitudes are discussed further in the following section.

\section{The Phenomenological Attitude}

The natural way of thinking, which fluctuates between assumptions, facts, and objectivity, is coupled with Husserl's (1931) phenomenological attitude. The researcher's phenomenological attitude philosophizes on a journey to discover the realness of everyday experiences (van Manen, 2014). Lopez and Willis (2004) stated:

An important component of Husserlian phenomenology is the belief that it is essential for the researcher to shed all prior personal knowledge to grasp the essential lived experiences of those being studied. This means that the researcher must actively strip his or her consciousness of all prior expert knowledge as well as personal biases. (p. 727) To discover the realness of human experience, I reflected on my everyday assumptions, biases, and preconceptions on experienced pediatric oncology nurses and their ability to use selfreflective practices (i.e., epoché). I then attempted to set those ideas aside through a process of bracketing (Moustakas, 1994). I used bracketing throughout this investigation to suspend judgment and foster a sense of wonder on the essence of self-reflection (Moustakas, 1994). According to Lopez and Willis (2004), in Husserlian phenomenology, there is the assumption that "there are features to any lived experience that are common to all persons who have had the experience .... referred to [as] universal essences" (p. 728). Understanding the essence invites the researcher to be open to a lived experience, "and thus, bracketing does not aim to change an individual's experience of the world, but to merely see it from a new light" (Christensen et al., 2017, p. 117). My goal of this qualitative study is to embody the phenomenological attitude and to view a human phenomenon with fresh eyes. 


\section{Embodiment of the Phenomenological Attitude}

I embraced a phenomenological attitude from the onset. For example, I reflected on my ideas and assumptions regarding nurses and their reflective practices. I first considered their unique experiences (i.e., the natural attitude): The natural attitude is described as an experience that "occurs within the context of the world that individual is engaged with" (Christensen et al., 2017, p. 115). I then set my natural attitude (i.e., "standpoint") aside to try to view their world in a new way (Christensen et al., 2017). Using the phenomenological attitude does not imply personal experiences and emotions are detached from the overall research process (Moustakas, 1994; van Manen, 2014). The phenomenological attitude is like an aperture through which I am better able to view the experiences of others without imposing my natural attitude.

\section{The Natural Attitude}

The natural attitude is comprised of "common sense everyday reality with the added belief in the existence of an external material reality, one which there is a straight forward acceptance of experience of knowledge" (Christensen et al., 2017, p. 115). For example, as I interact with the world, I may naïvely accept reality without scrutiny or deep reflection. Over time, the natural attitude becomes an intuitive and reliable realm of living where I often take for granted emotions, thoughts, judgments, dreams, and desires that are integral to my overall human experience (Christensen et al., 2017).

My natural attitude can be explained as being confident in the reliability of the nursing literature without finding research describing what self-reflection means to experienced nurses. While my natural attitude does not fully represent the direct experiences of nursing professionals, it can nevertheless be useful in guiding and establishing credibility for phenomenological research (van Manen, 2014). In the next section, I briefly describe my natural attitude (i.e., 
personal reflection), which is not meant to replace my subjectivity statement, revealed in the section titled, Rigor and Trustworthiness of the Study. The following section is an example of my natural attitude in the form of the researcher's subjectivity statement.

\section{Researcher's Subjectivity Statement}

I use reflective practices to interact and be present in my environment. My life consists of work as a certified athletic trainer and movement educator. Interacting with clientele, including nursing professionals, and caring for clients' specific needs requires that I have balance in my life and am truly present and attentive. My business is centered on addressing the movement patterns of all working professionals and athletes of all ages. I help athletes between the ages of 10 to 25 learn about themselves through movement and fitness. I also care for nursing professionals suffering from stress, fatigue, and mental and physical burnout. I treat nursing professionals mainly for back pain and joint pain that impede their ability to move effectively. In my facility, nursing professionals and athletes learn effective movement patterns for their needs and perform exercises that promote strength, flexibility, and injury prevention. I use selfreflective practices to connect with each client at a deeper level.

My life has been impacted by using self-reflection as an ongoing process. I am more aware of moments when caring for my own mental and physical health is essential to caring for the health of my clients. This reality sometimes means I share my feelings with clients and bond on a person-to-person level, although I am aware that showing vulnerability to clients is risky. Boundaries are important but need not be impediments to empathetic treatment. My hope is to inspire current nursing professionals to learn, reflect, and communicate their feelings in a professional manner. Healthcare professionals are sometimes not fully able to develop close 
relationships with patients for fear their emotions may impede medical objectivity. As a healthcare professional, I must remain objective and ethical with my patients.

I want patients to understand I am a human being first and treatment specialist second. I consider myself to be an authentic leader, as defined by Avolio and Gardner (2005). Being an authentic leader with patients carries risk because I may sometimes share too much personal information. As I reflect, however, I am aware that risking revealing my true self with patients is better than being emotionless and devoid of empathy and compassion.

Self-awareness is self-reflection in the moment. I currently work in an environment where professionalism is required, but professionalism grounded in human connection, not in separation or hierarchy. I use self-reflection to be more aware of moments when opening up and being reflective with clients is essential. There are also moments when being an objective medical professional is crucial. These qualities are a spectrum of care that is a mixture of empathy, compassion, and medical proficiency.

I have contemplated various ways to improve my treatment approaches to provide patients with quality care. When I self-reflect and combine thoughts and behaviors, the "I" (i.e., the ego) who is stressing about being stressed is no longer getting in the way of providing patients with care. Some of the self-reflective practices that help me cope with stress include maintaining fitness, enjoying music, art, and connecting with others in the process of care. My ability to manage stress has helped me to provide patients with the best quality care. I have created a successful business where I am in communion with my patients. For example, if I require my patients to walk with proper mechanics, I walk with them. If I require my patients to perform a certain movement pattern, I move with them. 
In essence, I am no longer a bystander contemplating what good or bad patient care is because I am moving and an integral part of the healthful life I am requiring of my patients. I am my own boss on this journey, and I have the capacity to affect change in the way I deliver patient care. Valuable experiences with patients and clients and the opportunity to develop my own operating principles as an entrepreneur have led me to believe the nature of patient care is being in the moment with, not for, my patients, as expressed by Hines and Gaughan (2014). Being with my patients is a form of self-reflection, and being immersed in the present moment helps me to eradicate worry and stress.

Self-reflection has transformed my pattern of thinking and behaving. Thinking about thinking, or the experience of using self-reflection, is what led me to develop this study. As a leader in my current setting, I realize there are still fragments of my experiences that continue to provide stress in my life. I am still reflecting on how to think and behave better to develop a long-term business centered on providing quality patient care. I believe there is a better way to connect with people and to provide them with the care they desire, not the care I believe they need.

I believe self-reflection is not just thinking about being better. The point is human beings have thinking minds, which can improve lives with conscious application. Self-reflection is a rebalancing or refocusing of the mind on what is naturally important, living better and not simply thinking about living better. For this reason, I wanted to learn how nurses make the time to use self-reflection while working in one of the most stressful settings - the pediatric oncology unit. My professional goals are to provide optimum care to my clients, to educate healthcare professionals to use self-reflection authentically, and to develop their emotional intelligence so 
they can communicate effectively to deal with the stress and burnout endemic in the medical environment.

In today's economy, there is a growing need to educate nursing students to prosper despite workplace demands, to deliver quality patient care, and to effectively communicate with patients, families, or colleagues who differ in age and levels of clinical experience (Enskär, 2012; Vogenberg \& Santilli, 2018). Vogenberg and Santilli (2018) stated, "The most difficult aspects of managing a workplace comprising of different generations of employees remains communication" (p. 49). Researchers have described pediatric oncology nurses as inadequately educated or ill prepared to effectively communicate their feelings with patients, families, and colleagues, a situation that creates an environment where nurses are susceptible to workplace stressors and feelings of burnout (Boyle \& Bush, 2018; Mukherjee, Beresford, \& Tennant, 2014). Nurses who experience perpetual feelings of burnout may eventually leave their roles for less stressful environments (Boyle \& Bush, 2018).

Self-reflection can be used to communicate difficult topics with patients and to reduce feelings of stress and burnout (Gelabert-Vilella et al., 2014; King-Okoye \& Arber, 2014; O’Neill et al., 2019; Rees, 2013). To begin this research, I focused my attention on describing the lived experiences of bedside nurses currently employed in pediatric oncology. This personal reflection is an example of my natural attitude. My natural attitude is important, but to fully embrace the phenomenological attitude, I needed to refrain from using the natural attitude to discover something new about self-reflection. Learning something new about self-reflection was accomplished by using the imaginative variation. 


\section{The Imaginative Variation}

The imaginative variation is a process "in which the person uses their imagination to change various features of the phenomena, by refusing to accept the initial meaning of the phenomena" (Christensen et al., 2017, p. 117). I was curious to imagine the possible ways in which pediatric oncology nurses use self-reflection and the meanings they attribute to its usage. Currently, ongoing self-reflection is part of the professional requirement of nursing professionals (Koshy et al., 2017). As I considered my natural attitude, I wondered how self-reflective practices in the consciousness of pediatric oncology nurses functioned considering nurses experience the suffering and deaths of children under their direct care. Koshy et al. (2017) discussed self-reflective practices for nursing professionals in their article and stated, "By reflecting you will develop your skills in self-directed learning, improve motivation, and improve the quality of care you are able to provide" (p. 1). This section has been a preview of my natural attitude. I discuss using the methodology of Husserl's (1931) TP and the phenomenological attitude in the following sections.

\section{Discussion of the Methodology of Choice}

Transcendental (descriptive) phenomenology is both a philosophy and a method of scientific inquiry first developed by Husserl to explore human experience (Husserl, 1931; Moustakas, 1994). This philosophy is ontological (what is the nature of being a human) and specifically seeks to ground the phenomena of human existence in terms of directed consciousness (intentionality) and objects of consciousness to explore the true nature of being in this world (Husserl, 1931; Moustakas, 1994; Sultan, 2018). Transcendental phenomenology is also an epistemological (How do we know our world as it really is?) method of inquiry that seeks to scientifically investigate the intentionality of human experience through genuine observation, 
one-on-one interviewing or group interactions, and deep reflection with human beings in their natural environment (Moustakas, 1994; Starks \& Trinidad, 2007). The following section will provide a general description of descriptive and interpretative phenomenology. These descriptions are in no way an exhaustive review and critique of the methodologies. Instead, the specific features of each phenomenological method are provided to illuminate the differences between the two.

\section{Husserl's (Descriptive) Phenomenology}

In descriptive phenomenology, the researcher engages in the epoché and phenomenological reduction to abstain from personal biases, preconceptions, and opinions on the phenomenon that is being explored (Lopez \& Willis, 2004; Moustakas, 1994). The epoché and phenomenological reduction are further discussed in this chapter. For Husserl, description of the participant's experiences is what constitutes the essence of a particular phenomenon (Moustakas, 1994). Lopez and Willis (2004) stated:

The belief that essences can be abstracted from lived experiences without a consideration of context is reflective of the values of traditional science and represent Husserl's attempt to make phenomenology a rigorous science within the prevailing tradition. This desire for scientific rigor underlies the use of the bracketing technique when doing descriptive phenomenology. (p. 728)

Although, the primary researcher may engage in epoché and bracket their biases and opinions, only the experience of participants is attained, which determines the essence of that particular phenomenon in Husserl's phenomenology (Moustakas, 1994). This distinction is different from Hermeneutic phenomenology (Moustakas, 1994). 


\section{Hermeneutic (Interpretive) Phenomenology}

Interpretive phenomenology is concerned with interpreting written text (Lopez \& Willis, 2004; Moustakas, 1994). According to Lopez and Willis (2004), "The word hermeneutic is derived from the name Hermes, a Greek god who was responsible for making clear, or interpreting, messages between gods" (p. 728). Researchers use hermeneutic phenomenology to attend to an interconnected human experience rather than focusing solely on knowing a specific experience (Charalambous, Papadopoulos, \& Beadsmoore, 2008; Heidegger, 1962; Lopez \& Willis, 2004; Solomon, 1987). Furthermore, the "focus of a hermeneutic inquiry is on what humans experience rather that what they consciously know" (Lopez \& Willis, 2004, p. 728). Moustakas (1994) stated, "Hermeneutic science involves the art of reading a text so that the intention and meaning behind appearances are fully understood" (p. 9). In hermeneutic phenomenology, as distinct from Husserlian phenomenology, the researcher and participants are not separate and therefore rich meaning can be gathered from the data (Moustakas, 1994). Additionally, using other methods of inquiry to analyze phenomenological data (e.g., social constructivist) is allowed in hermeneutic phenomenology (Lopez \& Willis, 2004). In Husserlian phenomenology, the researcher's main concern is to describe the essence of a participant's lived experiences. To accomplish this, the primary researcher engages in epoché and phenomenological reduction throughout the entire research process (Moustakas, 1994). How objects appear to the minds of participants is the main concern in Husserlian phenomenology.

\section{Objects That Appear to the Mind}

Edmund Husserl (1931) was mainly concerned with learning something new about human experience. Exploring lived experience is accomplished by reflecting and attending to the objects that appear to our mind (van Manen, 2014). What appears to the mind is the basis for 
relating to the world as an interconnected system. This concept is in stark contrast to the traditional ways of learning from objectivity and concrete reasoning. Transcendental phenomenology addresses unique concepts, which help researchers explore the abstract world of human experience. For example, when nurses observe objects, according to TP researchers, their minds are directed to those objects. The objects provide the mind with some form of information and meaning. To Husserl (1931), human beings have conscious minds. Possessing a mind inherently conscious and directed toward something outside of the mind can be a place to start learning about what an experience means. This meaning, or essence, is what Husserl believed was the manifestation of being.

Descriptive phenomenology and human experience. Understanding the essential features of human experience is what Husserl (1931) referred to as the essence. According to Moustakas (1994), "Husserl was concerned with the discovery of meanings and essences in knowledge. He believed that a sharp contrast exists between facts and essences, between the real and non-real" (p. 27). Learning something new about essence and being is complex and the feeling of being connected with the world is difficult to describe. Husserl focused on deep reflection and discovering meaning and knowledge from the mind's ability to direct consciousness toward objects (Moustakas, 1994), a term called intentionality, which is different from the English version of intention meaning a thing intended, an aim or plan (Lexico, 2019). In phenomenology, intentionality is the direct appearance of an object from the perspective of a human being. Husserl believed that objects had a certain quality that provides our mind with some form of information without prior knowledge and by directing and viewing objects in front of us (Moustakas, 1994). This state or quality of the mind to perceive and know about an object 
without prior knowledge (i.e., a pre-reflective, conscious mind, implicitly aware of objects) forms the basis for intentionality (Moustakas, 1994).

Intentionality. Investigating phenomena and the mind's intentionality is the central tenant of a phenomenologist; for example, what is our direct human experience of reality? (Christensen et al., 2017; Lopez \& Willis, 2004; Matua, 2015; Moustakas, 1994; Willis, Sullivan-Bolyai, Knalf, \& Cohen, 2016). Moustakas (1994) stated:

Intentionality refers to consciousness, to the internal experience of being conscious of something; thus the act of consciousness and the object of consciousness are intentionally related .... Knowledge of intentionality requires that we be present to ourselves and to things in the world, that we recognize that self and world are inseparable components of meaning. (p. 28)

Intentionality does not refer to the fact that the mind and the world are connected, but to the feeling and intuition of being interconnected with everything. Intentionality can be further understood as the innate ability of the mind to direct consciousness toward objects outside of the mind while developing subjective meaning from the intentional act (Christensen et al., 2017). Phenomenologists believe our minds are always directed toward some aspect of reality, always perceiving and excluding, transmitting and receiving information, which determines an experience and the meaning of those experiences (Moustakas, 1994; van Manen, 2014). The inside mind and the outside environment are moving together as a fluid process of intentional acts. Thus, to phenomenologist, the mind is directed toward objects.

The phenomenon of intentionality may lead to learning more about human experience by the meaning we derive from those objects. Nevertheless, intentionality remains a difficult concept to explain. Intentionality requires knowledge of two concepts, noema and noesis. To 
simplify these phenomenological terms, I provide an example of noema and noesis using the experience of a nurse perceiving a patient on the unit in the following sections (Christensen et al., 2017).

Intentionality and noema. When our minds are directed toward objects outside the mind, we first encounter the experience of that which appears in front of us (Christensen et al., 2017). For example, the noema can be a description of something that the nurse perceives when interacting with a patient (Christensen et al., 2017). The nurse perceives the patient. Any prior beliefs, feelings, or behaviors of the nurse relative to the patient do not matter at this moment. The nurse is looking at the patient as if for the first time. The nurse looks carefully at the patient for what seems to be a few seconds longer than usual. The nurse perceives the patient and this direct recognition is the phenomenon. Thus, noema is not the real or the objective aspects of the patient but the phenomenon of the nurse's mind directing and perceiving the patient (Christensen et al., 2017; Moustakas, 1994). Phenomenologists are concerned with researching the perception of the real without concerning themselves with the actual realness of an object. Intentionality is not only comprised of the mind's perceiving of objects but also with the incorporating of the meanings derived from our conscious experience of reality (Moustakas, 1994).

Intentionality and noesis. If noema is the mind perceiving something, then noesis is the process of having meaning from something we perceive (Christensen et al., 2017). Noesis is described as the phenomenon we experience when we direct our minds toward a particular object (Moustakas, 1994), for instance, a nurse reflecting and contemplating about the patient a little longer than usual. The example of the patient that the nurse sees may change in perception (noema) when the two interact (e.g., saying hello and waving a hand to the patient or moving closer to the patient to ensure the gestures are noted). The nurse's meaning of the perceived 
patient can remain the same or change through continuous experiences with the same patient. For example, the noema is the immediate meaning that the nurse associates with the patient (e.g., the patient seems to be suffering; I need to help; I see the mother is confused and does not understand the doctor's explanation).

The noesis, when compared to the noema, is much more meaningful than the initial perception (Moustakas, 1994). When nurses perceive patients on the treatment tables, they have immediate perceptions, and they are aware of the persons or objects. As a nurse takes a moment to reflect on the perception of the patient, the intentional act of perception may allow the nurse to notice that someone left the patient's room messy. The perception of the patient now has an experience that adds to the value and meaning of the patient (e.g., the experience of perceiving the patient is also the experience of experiencing a messy patient room). Therefore, the intentional act is not meaningful in itself when we merely perceive a person or object, which is the noema (Moustakas, 1994). In phenomenology, meaning and essence are derived from the continued exposure with the patient, which is the noesis (Moustakas, 1994). Intentionality is, therefore, a relationship between what our mind is directed toward and the meaning that directedness develops (Christensen et al., 2017). When the nurse reflects on the phenomenon of perceiving the patient, the nurse has added value to the story by reflecting on feelings on the messy room.

Noema and noesis. The noema and the noesis is, thus, a relationship between the perception of a person, or object, and the process of meaning we give to our perceptions (Moustakas, 1994). According to Moustakas (1994), “The 'perceived as such' is the noema; the 'perfect self-evidence' is the noesis. Their relationship constitutes the intentionality of consciousness. For every noema there is a noesis; for every noesis there is a noema" (p. 30). For 
example, when nurses perceive the patients on the treatment tables, they derive meaning from their experiences with them. Moustakas (1994) stated, "The working out of the noema-noesis relationship, the textural (noematic) and structural (noetic) dimensions of phenomena, and the derivation of meanings is an essential function of intentionality" (p. 31). In phenomenology, developing knowledge from the understanding of lived experiences can be accomplished when identifying, describing, and interpreting human intentionality. However, the concept of intentionally is difficult to explain when described as a static entity.

Intentionality as a living process. Intentionality can be considered a holistic process between objects that appear to the mind (perceptions) and the meaning (essence) evoked by that appearance (Christensen et al., 2017). When a nurse reflects on the patient and can see the patient appearing in the mind, this act of perceiving is the phenomenon of intentionality. The perception of the patient gives meaning later without the patient having to physically be present. The more the nurse reflects on the patient, the more layers of depth and meaning become unraveled. The immediacy of the mind to understand and identify with persons and objects, without having to think about them, is the phenomenon of intuition (Moustakas, 1994).

Intentionality and intuition. Intentionality and intuition are key concepts to the philosophy of TP. According to Moustakas (1994), "Husserl did not employ deduction in his transcendental philosophy; only intuition was used. Intuition is essential in describing whatever presents itself, whatever is actually given" (p. 33). In TP, intuition is what one's mind uses to immediately describe the essential features of objects that appear in human experience. Intentionality directs the mind while intuition is a feeling of knowing the essence of what is being experienced (Moustakas, 1994). To simplify the terms, intuition is the feeling and intentionality is the conscious experience of what is being perceived. 
Moustakas (1994) highlighted challenges of intentionality from the primary researcher's point of view: (a) explaining "the sense in which our experiences are directed" (p. 31), (b) recognizing noema, or the attributes of "consciousness that are essential for the individuation of objects (real or imaginary) that are before us in consciousness" (p. 31), (c) explaining noesis, or "how beliefs about such objects (real or imaginary) may be acquired, how it is that we are experiencing what we are experiencing" (p. 32), and (d) "integrating the noematic and noetic correlates of intentionality into meanings and essences of experience" (p. 32). I explore the challenges of explaining the process of intentionality further in the data analysis sections of Chapter 3.

In summary, TP is a way of exploring human experience. Deriving knowledge from the experiences of nursing professionals is accomplished through a process of intentionality. Intentionality is a structure of self-consciousness that incorporates both directedness of the mind toward objects (noema) and the process of meaning inherent in the things that are given in consciousness (noesis; Christensen et al., 2017; Moustakas, 1994). For instance, the minds of nurses are directed toward objects as they are using self-reflective practices in patient care. This is the noema of intentionality. Intuition and a stream of questions may begin to arise in the consciousness of nurses as they continue directing their minds toward the objects. The question then is: What do experienced pediatric oncology nurses perceive in patient care that stimulates using self-reflective practices? What do these nurses perceive and how does this act of perceiving lead to inquiry about their experiences of self-reflection? In TP, intuition is the ability to understand what is appearing in the mind instinctually and without conscious reasoning (Moustakas, 1994). 
There are challenges to describing the process of intentionality according to the methodology of Husserl's TP (Moustakas, 1994); therefore, steps are taken in the data analysis section to address these concerns. No matter how uniquely a nurse experiences a particular phenomenon (e.g., perceiving a patient on a treatment table), essential features of the overall experience can be described and interpreted as a meaningful human experience by the researcher. To learn something new, then, I must not focus heavily on my own intentionality of the phenomenon. According to the philosophy of TP, when I relinquish ideas and suspend prior judgments, I may begin to understand something new about a previously understood phenomenon. Learning and developing untapped knowledge are the main reasons I have chosen to use TP to explore human experience. I will move from the conceptual framework of TP to the methods of TP in the next section. The methods of TP help reveal information on the study of human essence and the methods of deriving knowledge from human experience, (a) the epoché (suspending judgment), (b) transcendental-phenomenological reduction (bracketing and returning to the experience), (c) the phenomenological interview process, and (d) the imaginative variation (Moustakas, 1994).

\section{Methods for Using Transcendental Phenomenology}

Modern qualitative research, other than that of phenomenology, does not require or demand depth into exploring the essence of human consciousness (Creswell, 2014; Moustakas, 1994). Going deep into the recesses of the mind requires exploration, induction, reflection, suspension of thoughts and judgment, and a return to the objects and experiences themselves to view the phenomenon anew (Moustakas, 1994). The process of temporarily reflecting and suspending thoughts and judgments on human experiences is called the epoché (Moustakas, 1994). The following example refers to the phenomenon of a nurse perceiving a patient on the 
treatment table. In accordance with phenomenology, if I am not thorough when reflecting on my own intentionality, personal experiences may get in the way of learning from others' experiences and may hinder my learning something new about the essential qualities of perceiving a patient on a treatment table (e.g., I see the patient, I reach for the patient, I place comforting hands on the patient. I know what a patient on a treatment table looks like so I do not need to investigate it further). Learning from experiences and reflecting on how I may or may not share certain qualities of that particular experience with others can be difficult. Philosophers and researchers have acknowledged the challenges in conducting transcendental phenomenological inquiry (Husserl, 1931; Moustakas, 1994; Starks \& Trinidad, 2007; van Manen, 2014). The book Phenomenological Research Methods by Moustakas (1994) helped to elucidate the step-by-step process of the TP methodology.

The step-by-step process begins with the epoché, is followed by the transcendentalphenomenological reduction, the phenomenological interview, and is concluded by developing the imaginative variation (Moustakas, 1994). The imaginative variation is the process of the researcher deriving meaning from the lived experiences of participants and describing the essence of the phenomenon in a written description. I use the imaginative variation to consider my ability to empathize with the unique experiences of participants. The process of creating the imaginative variation is detailed in the data analysis section. For this section, highlighting the epoché and the phenomenological reduction is paramount to understanding how this study was conducted.

Epoché. Epoché is a Greek word meaning, "to refrain from judgment, to abstain from or stay away from the everyday, ordinary way of perceiving things" (Moustakas, 1994, p. 33). In other words, the primary researcher uses the epoché to learn to see the world through a new lens 
by open-minded exploration into human experience. Learning to see the world anew is the main objective for using the methodology of TP. As noted in Chapter 2, little to no information can be found in the research literature on the experiences of pediatric oncology nurses and selfreflective practices. Transcendental phenomenology is a useful method of inquiry exploring human phenomenon that we know very little about. Moustakas (1994) stated, "In the Epoché, the everyday understandings, judgments, and knowings are set aside, and phenomena are revisited, freshly, naively, in a wide-open sense, from the vantage point of a pure or transcendental ego" (p. 33). Once I conducted the epoché—-learning to see the experience of using self-reflection anew-the next step was to accomplish the phenomenological reduction where I reflected upon my judgments and biases on the phenomenon and set them aside through bracketing. I then returned (i.e., reduction) to the phenomenon with a restored sense of wonder (van Manen, 2014). The phenomenological reduction encompasses both the epoché and bracketing. I discuss bracketing as a concept, along with the phenomenological reduction, in the next section.

Transcendental-phenomenological reduction. The phenomenological reduction is first viewed as a suspension of our own beliefs and opinions (Willis et al., 2016), which Husserl termed bracketing as a result from his background in mathematics (Husserl, 1931; Moustakas, 1994). I used bracketing to reflect upon my own experiences on the phenomenon of interest. While the epoché is learning to see the world with fresh eyes, bracketing is the actual process of suspending judgment (Moustakas, 1994). For example, I reflect and abstain from incorporating personal biases, beliefs, and opinions throughout the data collection process so that I may arrive at an authentic description of the phenomenon during the data analysis phases (Moustakas, 1994; Munhall, 2007). The phenomenological reduction was used to magnify (as a telescope) the full nature of human experiences, setting the stage for honest (unexplored) inquiry (Moustakas, 1994; 
Munhall, 2007). I describe my process of using the phenomenological reduction in the methods section of this chapter. Upon bracketing, I returned to the experiences of pediatric oncology nurses (phenomenological reduction) to describe the essence of self-reflection. I used the phenomenological interview as a method to collect data from the experiences of nurses.

The phenomenological interview and imaginative variation. Understanding particular human experiences that consist of thoughts, emotions, values, and perception in constant flux is a complex process (Moustakas, 1994). According to Moustakas (1994), “The phenomenological interview involves an informal, interactive process and uses open-ended comments and questions" (p. 114). In TP research, each participant is interviewed to obtain rich and detailed information. Once the phenomenological interview process is completed, the next step is to analyze and describe the data using the imaginative variation. In the methodology of TP, the imaginative variation helps the primary researcher imagine the phenomenon from different perspectives and vantage points. The imaginative variation is the process in which the researcher derives meaning from individual experiences and creatively describes the essence of the phenomenon in question in a write-up. The imaginative variation is performed several times until the essence of the phenomenon is revealed (Moustakas, 1994).

In summary, researchers use the conceptual framework of TP to explore lived experience and things as they appear to the mind using intentionality (noema-noesis) and intuition, the key concepts of TP. The methodology of TP involves the epoché, the phenomenological reduction (bracketing), the phenomenological interview, and the imaginative variation. By using TP, my experiences are directly connected to the ways in which this study was conducted and knowledge was obtained. Therefore, explicating my role as the primary researcher is integral to the methodology of TP. 


\section{Rationale for Choosing Transcendental Phenomenology}

Qualitative researchers attempt to provide a generalizable description of human experience so that the rigorous aspects of scientific inquiry remain intact (Creswell, 2014). Descriptive qualitative inquiry was used in this study to illuminate my personal reflective practice and internal dialogue on the movement of a central idea: I feel that I am independent of the world and, also, dependent on it. Lopez and Willis (2004) stated:

An assumption specific to Husserl's philosophy was that experience as perceived by human consciousness has value and should be an object of scientific study. Husserl believed that subjective information should be important to a scientist seeking to understand human motivation because human actions are influenced by what people perceive to be real. (p. 727)

I chose TP as an investigative tool because TP affords a scientific process to guide the search to understand human experience and the ways ideas or beliefs influence behaviors. By choosing TP as a qualitative framework and using objectivity as a researcher (epoché), I may begin to understand how people experience similar events differently and how they describe the meaning of their experiences. For example, nurses from different cultural backgrounds may describe the essence of their self-reflective practice experience differently even if they live and work in the same social setting. According to Carel (2011), "Phenomenology understands perceptual experience as embedded in a particular culture and as having a particular meaning, based on the concepts and values of the culture" (p. 35). In phenomenological inquiry, human beings have one thing in common and that is their self-conscious mind perceiving and forming meaning and knowledge (Moustakas, 1994). The essence of human experience, despite being affected by cultural differences, has the potential to remain whole and unbroken. 


\section{Describing Human Essence}

I focus on understanding the essence of human experience in this study. I was interested in understanding the essential features and structures that create conditions for self-reflection in the clinical setting and the features specific to the clinical setting that influence nurses to use or not to use self-reflection. Transcendental phenomenology was chosen because researchers can find a qualitative method to explore human experiences that remain unexamined, such as pediatric oncology nurses and self-reflective practices.

Pediatric oncology nursing is considered a specialty. In this specialty, workplace stress may be intensified by the frequency of young patients suffering or losing their battles with cancer-related illnesses (Zander et al., 2010, 2013). The existing literature surrounding this specialty includes limited information on two topics of interest: (a) the experiences of nurses with 10 or more years of bedside experience and (b) how these experienced nurses use selfreflective practices in their patient care to minimize the negative effects of workplace stress.

\section{Deriving Knowledge From Lived Experience}

According to Reid (2013), it is important to understand and to research the lived experiences from the nurses' perspectives. Nurses working in critical environments, where young children suffer or lose their battle with cancer and die, report symptoms such as posttraumatic stress disorder, similar to that of combat soldiers (Jonsson \& Halabi, 2006; Laposa, Alden, \& Fullerton, 2003). Understanding the lived experiences of such nurses may impact a wider population such as student nurses, novice nurses, nursing educators, patients, and their families. Currently, learning how pediatric oncology nurses use self-reflective practices to mitigate the effects of stress and burnout remains unexplored. Previous studies (i.e., Charalambous \& Kaite, 2013; Enskär, 2012; Hines \& Gaughan, 2014; Mirlashari et al., 2017) do 
not provide adequate examinations of the experiences of pediatric oncology nurses who use selfreflection in clinical settings. Learning about these experiences requires the researcher to develop, in ethical manner, rapport and trust with each participant (Moustakas, 1994). The following is a discussion of the research ethics and descriptions of the methods used to develop rapport and trust with the study's participants.

\section{Discussion of Research Ethics}

The Institutional Review Board (IRB) of Chapman University approved this study prior to my recruitment of participants and data collection. Once participants were selected, they were asked to read the IRB-approved participant consent form (see Appendix A). I was available, either by phone or in person, to answer any questions participants had on the nature, purpose, and requirements of this study as recommended by Moustakas (1994). Information pertaining to the confidentiality of the data was provided to each participant. Moustakas (1994) stated, confidentiality is "maintained relevant to the data to be used unless the participant was fully informed and agreed to its use" (p. 110). Participants were free to end their participation at any time and without consequences.

\section{Developing Rapport and Trust}

Rapport and trust between participants and me was essential for this type of phenomenological research. Developing an authentic and trusting relationship with participants helped me gather a rich description of their lived experiences. Sultan (2018) stated:

Building rapport and trust is not about going through the motions of forcing relationships through false shows of warmth and connection. It is about being transparent with regard to all aspects of the research study in which you have invited these individuals to 
participant. It is also demonstrating congruence between what you say and what you do.

(p. 118)

Cultivating rapport and trust among all participants was maintained throughout the entire study by three methods: (a) maintaining communication with each participant and answering any questions or concerns, (b) being available to interview each participant in environments that were conducive to describing their emotional stress or wellbeing, and (c) respecting the dignity of their experiences by maintaining confidentiality, listening carefully, and being attentive to their specific emotional/physical needs (Moustakas, 1994).

\section{Rigor and Trustworthiness of the Study}

Although Moustakas (1994) does not delineate between rigor and trustworthiness (validity) of phenomenological data, I used Bailey's (2007) book, A Guide to Qualitative Field Research, to differentiate rigor and trustworthiness of the data—specifically, credibility. Furthermore, to increase trustworthiness of phenomenological data, a detailed description of the epoché, bracketing, and phenomenological interview has been described. According to Bailey (2007), "Trustworthiness requires conducting and presenting the research in such a way that the reader can believe, or trust, the results and be convinced that the research is worthy of his or her attention" (p. 181). To further enhance trustworthiness of the data, I included three strategies, member checking, peer debriefing, and the researcher's subjectivity statement (Bailey, 2007; Creswell, 2014; Moustakas, 1994). The researcher's subjectivity statement was used to explicate the reasons for conducting phenomenological research and to delineate biases, presuppositions, and viewpoints that were bracketed throughout the study. 


\section{Credibility (Internal Validity)}

In phenomenological research, the researcher uses credibility to indicate the data were collected and analyzed appropriately and rigorously (Bailey, 2007). I describe the data collection methods in great detail. The data analysis was conducted using Moustakas' (1994) methods for analyzing data (i.e., modified Stevick-Collaizzi-Keen). These steps are provided in the following sections. Another key aspect of adding credibility to the data is the personal experience of the primary researcher (Bailey, 2007). For example, to ensure that the experience of the primary researcher was not detrimental to the credibility of the study, I kept field notes throughout the data collection and data analysis phases to keep a record of the epoché and the process of bracketing. Furthermore, member checking, peer debriefing, and a semi-structured interview guide were used to add credibility (see Appendix B). To ensure data reliability, the interview guide was developed with the help of an experienced pediatric oncology nurse and an expert in qualitative research. According to Bailey (2007), "Reliable questions are those that, regardless of when they are asked, elicit the same responses from interviewees. Reliable respondents are those who provide consistent answers" (p. 184). To ensure reliability, each participant was given the interview guide prior to the initial interview. This effort was conducted to ensure that participants had adequate time to think deeply about their responses and to provide vivid accounts of their lived experiences.

\section{Member Checking}

Member checking is a process whereby each participant has the opportunity to review his or her data, verify statements, and make changes, corrections, or clarifications during the second and third interviews (Creswell, 2014). Each participant was integral to ensuring the validity and authenticity of the information gathered in this phenomenological inquiry (Moustakas, 1994). 
Furthermore, Bailey (2007) stated, "For an analysis to be considered successful, it must ring true with two specific segments of the researcher's audience: (1) members of the setting and (2) colleagues who are experts on the topic" (p. 185). Participants received their individual transcripts (three in total) for member checking. I did not receive feedback via email or phone from any participants to correct or clarify their transcripts. Prior to the second and third interviews, each participant was asked if he or she wanted to change, correct, or clarify any aspect of the interview transcripts. No participants provided in-depth feedback during memberchecking rounds; all but one participant read their transcripts and requested corrections or additions. These edits were minimal, mostly requiring changes in medical terminology. A total of 18 transcripts were generated, three from each participant.

\section{Peer Debriefing}

Peer debriefing occurred with a qualitative expert, my research advisor, who was available weekly to discuss concerns, questions, or ideas I had on the study. This debriefing included, but was not limited to, discussions on data collection, data analysis, and techniques for writing the final manuscript. My research advisor helped me establish a strong foundation for conducting phenomenological research by being a supportive research mentor from the onset. Peer debriefing allowed me to obtain valuable feedback from a qualitative expert and generating trustworthy data.

\section{Research Methods and Design}

I described the philosophy and methods of TP in the previous sections. This section is an in-depth discussion of the research methods and design. I describe the setting, inclusion criteria, and the population and sampling procedures in the next section. 


\section{Setting}

All participants were recruited from a 33-bed pediatric hematology oncology stem cell transplant intensive care unit in a Southern California teaching hospital serving a large metropolitan area. This unit is dedicated to providing specialized care to children from birth to age 21 diagnosed with pediatric blood-related diseases and cancer (e.g., leukemia, brain tumors, and diseases such as hemophilia).

\section{Inclusion Criteria}

All participants were required to have at least 10 or more years of bedside experience and use self-reflective practice. Each participant received the interview guide prior to the first interview. The interview guide contained a brief discussion of self-reflective practices to help participants understand the context of this study (see Appendix B). Six participants consented to participate. Participants were RN-certified to use pediatric cancer treatment protocols. Registered nurses are uniquely qualified to provide patient and family support during every phase of care, illness, diagnosis, treatment, and end-of-life. I focused on recruiting nurses who continuously provided care for 10 or more years. The selection of individuals enabled the comparing and contrasting of the experiences of nurses in similar working environments, and provided multiple perspectives (Bailey, 2007).

Participants were recruited based on their willingness to share their direct experiences, including the topics of suffering and death. Due to the sensitive nature of this study, steps were taken to ensure respect for participants and ethical handling of information (Bailey, 2007). Another method used was to display empathy and compassion when participants cried or showed distress after sharing an intimate moment. I made sure to stop the interview and pause the audio recording whenever I felt it was necessary to be with the participant and share my condolences. 
The interview and audio recording resumed once the participants were ready. No interviews were completely stopped or rescheduled due to the emotional nature of the subject matter. I made sure always to immediately offer comfort and reassurance that no narratives about their patients would be in the interview transcript without their approvals. All participants felt comfortable enough to continue their interviews once they had a moment to recover.

Sampling technique. I used a purposeful sampling technique to recruit nurses (Maxwell, 2005). According to Maxwell (2005), purposeful sampling is "a strategy in which particular settings, persons, or activities are selected deliberately in order to provide information that can't be gotten as well from other choices" (p. 88). This sampling technique was a flexible approach to locating, recruiting, and identifying participants who would provide a rich description of the phenomenon of interest. This type of sampling was also a feasible recruiting approach useful for the qualitative nature of this study.

Locating participants. Access to each participant was organized through Amanda, a friend of the researcher. As a CNS and as part of the support staff on the unit, Amanda was interested in in my gaining access to a group of nurses employed on her unit. Amanda identified eight nurses who fit the inclusion criteria: 10 or more years of experience and use self-reflective practices. I discussed inclusion criteria with Amanda, and I emphasized the need for participants able to provide rich details on self-reflection. I also provided Amanda with the interview guide. During the interview process, Amanda was in the process of transitioning from the pediatric oncology unit to the neonatal intensive care unit (NIC-U) and passed along my contact information to nurses she felt would benefit from participating in this study. Purposeful and snowball sampling strategies were useful considering the timeframe of this study. Once I contacted nurses interested in participating, I reassured them there were no consequences to not 
participating in this study or in choosing to drop out at any time. I am not directly connected with their hospital and have no authority to require their participation.

Snowball sampling. The IRB-approved recruitment emails were sent to eight nurses initially identified as potential participants. Only four responded with intent to participate in this study: Melissa, Beth, Emily, and Nicole. Amanda was the first who consented to participate. The sixth participant, Lucy, was obtained through a snowballing process of recruiting. Sadler, Lee, Lim, and Fullerton (2010) stated, "After the initial source helps to recruit respondents, the respondents then recruit others themselves, starting a process analogous to a snowball rolling down a hill" (p. 371). Beth, who had already consented to participate in the study, gave my contact information to an experienced nurse who fit the inclusion criteria. Beth connected me with Lucy, who texted me with her intent to participate. After I discussed the details of this study with Lucy, she consented to participate. Snowball sampling is advantageous but can also be detrimental to the overall generalizability of results by increasing the chances for researcher bias or "over-representation of individuals with numerous social connections who share similar characteristics" (Sadler et al., 2010, p. 3). Nevertheless, snowball sampling was essential to the timeline of this study. Participants shared their experiences with other nurses who fit the inclusion criteria. The nurses who fit the inclusion criteria were able to directly connect with the researcher, an outcome perhaps not possible using other methods of recruitment.

Participants' demographic information. Only first names of participants were saved on my password-protected computer, and I provided them with pseudonyms for confidentiality once the interview transcripts were created (see Table 1). Phone numbers and emails were gathered to maintain communication with each participant throughout the research process and were saved 
Table 1

Participant Demographic Information

\begin{tabular}{ccccc}
\hline $\begin{array}{c}\text { Participant } \\
\text { Pseudonyms }\end{array}$ & Age & $\begin{array}{c}\text { Pediatric Oncology Nursing } \\
\text { Experience }\end{array}$ & $\begin{array}{c}\text { Total Nursing } \\
\text { Experience }\end{array}$ & $\begin{array}{c}\text { Highest Level of } \\
\text { Education }\end{array}$ \\
\hline Amanda & 37 & 14 & 14 & Doctor of Nursing Practice \\
Melissa & 41 & 18 & 18 & Bachelor's in Nursing \\
Beth & 51 & 30 & 30 & Bachelor's in Nursing \\
Emily & 56 & 22 & 22 & Associates in Science \\
Nicole & 46 & 18 & 18 & Master's in Nursing \\
Lucy & 57 & 36 & 37 & Bachelor's in Nursing \\
\hline
\end{tabular}

on a password-protected laptop. Demographic information also included age, years of pediatric oncology nursing experience, years of total nursing experience, and highest level of education.

\section{Data Collection Procedures}

The interview guide was developed with the approval of a nursing professional (i.e., Amanda) and an expert in qualitative research (i.e., dissertation chair; see Appendix B). The interview guide contains semi-structured and open-ended questions. To obtain richness and depth from the direct lived experiences of participants, probing questions were used during each interview exclusively for each participant. To stimulate self-reflection, the interview guides were provided to participants at least 2 weeks before the first interviews.

Following the first interviews, probing questions were added to the interview guide, such as (a) How would you define self-reflective practices? and (b) What is self-reflection an experience of? These two questions were provided to each participant at the time of the second interview. The two questions were reiterated at the beginning of the third and final interview if clarification needed to be obtained from participants.

\section{A Structure for a Phenomenological Interview}

The interview process was guided by Bevan's (2014) structure of phenomenological interview according to Husserl's phenomenological theory, which includes elements pertaining 
to me as the primary researcher (e.g., the phenomenological attitude and approach) and to a structure for developing interview questions. In the phenomenological attitude, I am to remain authentic in describing the direct experiences of each participant and must accept the ways they describe their worlds (Bevan, 2014). The primary researcher's phenomenological attitude is derived by the phenomenological reduction (epoché). Bevan (2014) identified three elements: (a) “acceptance of natural attitude of participants" (p. 139), (b) "reflexive critical dialogue with self as the primary researcher" (p. 139), and (c) “active listening with each participant” (p. 139).

The phenomenological structure of developing interview questions is arranged in three categories, contextualization, apprehending the phenomenon, and clarifying the phenomenon (Bevan, 2014). Using Bevan's (2014) structure of phenomenological interviewing, example questions from the interview guide were (a) contextualization or eliciting the life world in natural attitude: "Tell me about how you came to be employed or interested to work at this particular hospital/unit" and "Tell me your experience on a typical day in the hospital/unit"; (b) apprehending the phenomenon or modes of appearing in natural attitude: "As a nurse, tell me about a patient care experience(s)" and "Describe the type(s) of individual self-reflective practices you use to manage stress"; and (c) clarifying the phenomenon or meaning through imaginative variation: "Do you believe self-reflective practices would play an important role for novice nurses? If so, in what ways?” (see Appendix B for the questions used for each category).

\section{Initial Contact}

I met with each participant, individually and face-to-face for the first in-depth interview. These meetings helped me provide participants with rich descriptions of both the phenomenological research design and data collection procedures unique to my study.

Additionally, I was able to describe why this type of research is important to me as a non-nursing 
professional. I shared my passion for this study during the first interactions to develop context for why I was choosing to explore self-reflection among nursing professionals.

\section{Location of Interviews}

Participants had the autonomy to choose the locations of each interview (Moustakas, 1994). Interview locations ranged from local coffee shops to one participant's home. Each location was intended to be away from the clinical setting. One participant, Nicole, requested the final interview to be scheduled in her office, located on the unit. Nicole was in a unique situation in her role as a patient and family educator; she worked from a private office. The final interview with Nicole occurred in her office where she was able to be candid about her experiences.

\section{The Initial and Follow-Up Interviews}

Each of the six participants completed a total of three, in-depth, semi-structured interviews. The first interviews were the longest followed by two follow-up interviews, considered member-checking rounds. The initial interview was essential to phenomenological research to enter a person's world and gather detailed information about the self-reflection phenomenon (Moustakas, 1994; van Manen, 2014). All interviews were audio-recorded using a digital audio-recording device (Sony ICD-UX560 digital voice recorder). Questions or concerns were addressed prior to recording. Once the participant was ready to begin the interview, the recording device was turned on, and I began the interview with "Welcome and thank you for participating in this study.”

\section{Transcribing Interviews}

Audio data were transcribed immediately after each interview using an online transcription service, Rev.com. According to Bucholtz (2000), there is a distinct difference between the spoken and written word. It is necessary to obtain a clear understanding of what 
participants are saying versus what is transcribed or interpreted by the researcher to gather authentic representation of a lived experience. Bucholtz (2000) described two types of transcriptions: (a) naturalized and (b) denaturalized.

Naturalized transcriptions retain "written discourse conventions" while denaturalized transcriptions retain "links to oral discourse" (Bucholtz, 2000, p. 1439). A naturalized transcription approach was used in this study. Naturalized transcripts "run the risk of failing to call enough attention to linguistic form and its transformation from speech to writing" (Bucholtz, 2000, p. 1461). Only repeating words such as "um," "like," and "you know" were deleted. These words are considered denaturalized by Bucholtz (2000) and are the only ones that I deleted to clarify. Bucholtz (2000) stated, "Denaturalized transcription, in its faithfulness to oral language, may make speech itself seem alien" (p. 1461). The naturalized transcription process used in this study makes each transcript easier to read and analyze. For example, sentences are formatted as part of a list of complete sentences. Listing each sentence was helpful for organizing the data because I was able to read and analyze each sentence separately.

Once transcripts were completed and saved in a Word document, they were emailed to participants for member checking. To ensure participants received copies of their transcripts, I began each follow-up interview with an opening request, "You were sent an email that included your interview transcript. At this time, please share any comments, ideas, or concerns you may have had after reading your transcript." I wanted participants to understand their opinions mattered to the process.

\section{Process of the Interviews}

The first interview began with a social conversation "aimed at creating a relaxed and trusting atmosphere" (p. 114) as proposed by Moustakas (1994). I did not initiate the interview 
with the first question of my interview guide. The aim was to create a trusting space by asking questions not directly connected to the phenomenon such as "How was your day?" I followed up the opening conversation by asking the participant to "take a few moments to focus on the experience, moments of particular awareness and impact, and then to describe the experience fully” (Moustakas, 1994, p. 114). It was my responsibility to create a safe atmosphere for each participant so they felt comfortable enough to describe experiences honestly in rich detail (Moustakas, 1994). Interviews lasted approximately 1 to 1.5 hours. The subsequent interviews lasted an hour or less due to the abundant data already gathered from the initial interviews. With 18 audio-recorded interviews, there were approximately 21 hours of audio-data transcribed.

\section{Bracketing Throughout Data Collection}

As previously stated, prior to conducting the interviews with participants - in accordance with TP methodology-I engaged in the process of the epoché by suspending any biases or preconceptions of the phenomenon. I later returned to the phenomenon through the process of the phenomenological reduction (bracketing). Bracketing occurred when I set aside my thoughts and opinions on what I already knew about using self-reflection to revisit the phenomenon with an open mind (Bevan, 2014). Epoché and bracketing occurred throughout the entire interview process. During the interviews, I remained engaged by listening, making eye contact, and writing notes in my journal as opinions and biases arose. In phenomenology, writing notes in a journal is a practical method to bracket concepts, ideas, or biases and to separate them and suspend judgment during the data collection and analysis phase (Moustakas, 1994; van Manen, 2014). Judgments may be in the form of thoughts or ideas, an opinion or experience, or even a question on the phenomenon. Writing in the journal was kept to a minimum during the recorded 
interviews. Bracketing occurred prior to or immediately after each interview was completed because I wanted to spend time engaging with participants rather than writing my thoughts.

\section{Data Analysis Method}

The data were analyzed using the Stevick-Collaizzi-Keen method, as described by Moustakas (1994). Moerer-Urdahl and Creswell (2004) suggested the "systematic procedures and detailed data analysis steps as outlined by Moustakas are ideal for assisting less experienced researchers" (p. 21). Furthermore, the Stevick-Collaizi-Keen method was chosen for two reasons. The step-by-step process was not difficult to comprehend, and the method has been proved to be an effective approach for analyzing phenomenological data (Moerer-Urdahl \& Creswell, 2004; Moustakas, 1994). The Stevick-Collaizzi-Keen method has four distinct steps "in the appropriate order of analysis" (Moustakas, 1994, p. 121). The first step relates specifically to the primary researcher's lived experience on self-reflective practices (Moustakas, 1994). With help and guidance from a qualitative expert and research advisor, I decided to further modify the Stevick-Collaizzi-Keen method by refraining from using my own experience of the phenomenon as integral to the data analysis process. Researchers using Husserlian phenomenology seek to describe the essence of experience from the participant's perspective (Moustakas, 1994). In contrast, interpretive phenomenology does allow for the researcher's experience to be part of the data analysis process (Lopez \& Willis, 2004). Although modifications were made to the Stevick-Collaizzi-Keen method, the first step that pertains to the primary researcher can be considered the researcher's subjectivity statement. The researcher's subjectivity statement is not part of the data analysis, but it does provide a description of my experiences using self-reflection. In the following section, I discuss the modifications made to the data analysis method. 
Modifications to the data analysis method. There are four steps for organizing and analyzing the data (Moustakas, 1994). Steps 1 and 2 pertain solely to the primary researcher's experience. As previously stated, Step 1 is considered the researcher's subjectivity statement and not part of data analysis. Step 1 consists of "using the phenomenological approach [to] obtain a full description of your own experience of the phenomenon" (Moustakas, 1994, p. 122). Indirectly, the primary researcher conducts Step 2 to complete these steps:

(a) Consider each statement with respect to significance for description of the experience, (b) Record all relevant statements, (c) List each nonrepetitive, nonoverlapping statement, these are the invariant horizons or meaning units of the experience, (d) Relate and cluster the invariant meaning units in to themes, (e) Synthesize the invariant meaning units and themes into a description of the textures of the experience. Include verbatim examples, (f) Reflect on your own textural descriptions and, through imaginative variation, construct a description of the structures of your experience, and (g) Construct a textural-structural description of the meanings and essences of your experience. (Moustakas, 1994, p. 122) During the entire data analysis process, the primary researcher is exposed to Step 2 and interacted with each of the seven steps while engaging in bracketing.

Qualitative researchers have argued refraining from biases and preconceptions (i.e., epoché) on particular human phenomenon is impossible (Lopez \& Willis, 2004). I believe I am reflected in the data because I was integral to data collection and analysis. Therefore, Steps 1 and 2 were not completely excluded from data analysis. Instead, highlighting the philosophical underpinnings of Husserlian phenomenology (i.e., epoché and phenomenological reduction) was critical for developing a qualitative study framed by descriptive phenomenology (Lopez \& Willis, 2004; Moustakas, 1994). 
I used a modification of the Stevick-Collaizzi-Keen method to focus on data analysis, specifically, Steps 3 and 4 (i.e., the participants' experiences). Step 3 is, "from the verbatim transcript of the experience of each of the other [participants], complete the [previous] steps, a through g" (Moustakas, 1994, p. 122). After I completed Step 3 for all participants, the final stage of analysis was to conduct Step 4. Step 4 is "from the individual textural-structural descriptions of all [participants'] experiences, construct a composite textural-structural description of the meanings and essences of the experience, integrating all individual texturalstructural descriptions into a universal description of the group as a whole" (Moustakas, 1994, p. 122). The following sections discuss my completion of Steps 3 and 4 in depth.

Step 3a: A detailed description of data analysis. In Step 3a, I read the transcripts of each participant from my laptop three times in full and without taking notes. During the third round of reading, I italicized words and key phrases that stood out. For example, any words that pertained to the experience, or words participants used, such as self-reflection, reflection, or reflect, were italicized. The italicized words helped synthesize my understanding when I returned to read the data a fourth time.

Step 3b: Record all relevant statements. To complete Step 3b, I read the transcripts a fourth time. The fourth round of reading was conducted on hard copy allowing me to make margin notes. During the fourth round of reading, I identified significant statements, those directly related to self-reflection, using colored pens and highlighters. The colors helped me identify each significant section and to visualize my reflective thought process. I chose to use this method of analysis instead of data analysis software because I wanted to feel connected to participants by reading transcripts in hand. All transcripts of Interviews 1, 2, and 3 were printed out on $8.5 \times 11$-inch copy paper and organized in binders for participants. After finishing the 
initial coding in Step 3b (i.e., highlighting significant statements), I returned to read each transcript for a fifth time. The fifth round of reading was conducted to further review and understand particular sections that I had previously emphasized and to review how they related to the phenomenon.

During the fifth round of reading, I identified significant statements, an exercise Moustakas (1994) referred to as invariant horizons or meaning units. I use the term meaning units to also refer to invariant horizons because they are used interchangeably by Moustakas (1994). Meaning units were identified from the transcripts of each participant. For example, definition of self-reflective practices was considered a meaning unit. I asked each participant to define self-reflective practices using their own words. This particular question was helpful in developing meaning units. Although each participant provided different definitions, I grouped their responses into one meaning unit. This process of grouping was similar for most of the questions that I asked. For instance, questions and responses from the interview guide were included in the transcripts of each participant. Each response may have created additional meaning units; however, not all meaning units were derived from each question. One particular meaning unit was being a professional as some participants described their experiences of being professional and using self-reflection as integral to their clinical practice. Being professional was not particular to any question; rather, it was identified as a meaning unit during data analysis.

Step 3c: Listing the meaning units. Step 3c was listing each nonrepetitive, nonoverlapping meaning unit (Moustakas, 1994). After identifying the meaning units, I created a new Word document and generated a list of the meaning units using direct quotes from the original transcripts. I only kept the meaning units that I identified as significant in this new document. This process was completed for each participant. Three interview transcripts and 
significant meaning units were combined into one document for each participant. From the 18 transcripts, I was now working with six documents (e.g., one lengthy document for each participant), which contained only meaning units (supported by direct quotes). Sections that pertained to specific experiences, or to specific questions, were grouped in a manner helpful for identifying initial themes. As I continued to visualize the connections between each unique experience, meaning units began to generate preliminary themes for each transcript. Moustakas (1994) referred to this process as horizonalization, or laying out all of the data and analyzing it equally.

Steps $3 \boldsymbol{d}$ and $3 \boldsymbol{e}$. Step $3 \mathrm{~d}$ was to relate and cluster the meaning units into themes. Themes were generated in terms of their characteristics and how they addressed the research question. For example, a theme was generated and given the label I'm Part of It about how a participant felt about using self-reflective practices. Once I had added all the meaning units and placed them into clusters of themes, I combined themes to develop a textural description. Textural descriptions include only direct quotes that pertain to the experience of using self-reflective practices (Moustakas, 1994). The process of adding direct quotes to each theme was considered to fulfill Step 3e as I synthesized the meaning units and themes into a description of the textures of the experience (Moustakas, 1994). Textures describe the what of human experience in relation to the phenomenon (Moustakas, 1994). Step 3e required significant time with the data to explore how my original ideas were transforming. I wanted to make sure I did not become overly critical during this part of data analysis. I remained open to the phenomenological reduction and continued to be open to the experiences of each participant.

Step $3 f$ and $3 g$. Step $3 \mathrm{f}$ was to reflect on the textural descriptions I had created and to begin the imaginative variation process. Through imaginative variation, I developed an outline of 
the structural descriptions (Moustakas, 1994) for each participant. Researchers use structural texts to describe the context or setting that influences how participants experience the phenomenon of self-reflection (Moustakas, 1994). In Step 3g, I combined the textural-structural descriptions that represent the essence of using self-reflective practices. The textural-structural descriptions were created for all six participants. Step 3 was now complete, and I moved on to the final step of data analysis.

Step 4. In Step 4, I combined all the textural (themes and direct quotes that represent the experience) and structural (direct quotes that provide context to the experience) descriptions of each participant into one lengthy document. This final Word document, which contained all the relevant data, was shared with my research advisor. Once peer debriefing was completed, I developed a codebook that contained 115 meaning units and direct quotes (see Appendix C). These meaning units were grouped into 26 ideas, which were further analyzed using the imaginative variation to expose the essence of using self-reflective practices. I revealed the essence of pediatric oncology nurses' experiences using self-reflective practices in the clinical setting during the final step of analysis. I discuss results of the study in depth in the next chapter. A summary of findings are arranged in three sections: (a) setting the stage, (b) the experience of using self-reflective practices in patient care, and (c) the experience of using self-reflective practices away from the clinical setting. 


\section{CHAPTER FOUR - RESULTS}

In Chapter 4, I present results of the study in accordance with the research objectives. The main objectives of this study are to describe (a) how experienced pediatric oncology nurses develop an understanding of using self-reflective practice, (b) how experienced pediatric oncology nurses use self-reflection in the clinical setting, and (c) how using self-reflection in patient care may impact the wellbeing of experienced nurses. Chapter 4 is arranged in three sections: (a) setting the stage, (b) using self-reflection in the clinical setting, and (c) using selfreflection away from the clinical setting. In phenomenological research, it is not essential to understand why nurses use self-reflection. Instead, phenomenology is concerned with the nurses' experiences of using self-reflective practices. I provide information that gives context and meaning to participants' experiences in Section 1. In Sections 2 and 3, I provide vivid portrayals of participants' experiences using self-reflective practices.

I found experienced pediatric oncology nurses use self-reflection to develop better ways of interacting with others in the clinical setting. By interacting with patients, families, and colleagues, participants of this study used self-reflection as a catalyst to learn about empathy, compassion, and camaraderie. Participants actively engaged in reflective behaviors during and after their shifts; however, they reported inadequate time during their shifts to use self-reflective practices consistently. Pediatric oncology nurses are trained to provide care to critical patients. Understanding the experiences of nurses who use self-reflection in the clinical setting requires knowledge of the environment they work in. In Section 1, I will detail how participants (a) defined self-reflective practices, (b) described the advantages and consequences of using selfreflective practices, (c) described how they learned about using self-reflective practices, and (d) described making time for clinical-based reflective practice. What emerges from the data is a 
need for nurses to set the stage by describing why they use self-reflection and how they develop an understanding of reflective practice in the clinical setting.

\section{Section 1: Setting the Stage}

The pediatric oncology nurses in this study explained how caring for critically ill patients is demanding work, which often leaves them feeling overwhelmed, stressed, helpless, and with little to no energy left to care for their wellbeing. For example, when caring for patients who are not responding well to their treatments, participants described it was difficult to be consciously aware of anything other than their patients' health. Participants clarified stopping to use selfreflection is challenging when they are responsible for maintaining the health of multiple patients requiring specialized care. Melissa stated, "You can't stop, especially if you have other patients" (personal communication, September 2, 2018). Beth said, "The moments are few because I'm always needed for 12 hours. I don't really have the time to go deep into thought because everyone needs me" (personal communication, December 4, 2018). During the interviews, each participant described the struggles to find time to self-reflect; nevertheless, they all shared their definitions of self-reflection.

\section{Defining Self-Reflective Practices}

Understanding what participants mean by self-reflective practices is critical to understanding how self-reflection appears to nurses in the context of the clinical setting. To Amanda, self-reflection is

being able to pause and think through the internal experience that you have in a scenario. Self-reflection is an internal process. It's not just looking at the scenario. It's looking at how I felt about the scenario. It's my experience. (personal communication, October 19, 2018) 
For Amanda, self-reflection is the ability for the mind to temporarily stop to sense how she is feeling in relation to what she sees. Using self-reflective practices appears to Amanda as an internal experience that she perceives as her own; "being able to pause and think" is a mental activity that is necessary for Amanda to use self-reflective practice in the clinical setting.

A connection between the inner and outer world. Nicole described self-reflection as "taking the opportunity, for myself, to process emotionally, mentally, and even physically the experience that just occurred" (personal communication, November 16, 2018). Similar to Amanda, Nicole defined self-reflective practices as an internal act that seeks to take hold of the inner and outer network of human experience. Participants described self-reflective practices as an act that combines the internal and external characteristics of human experience. For example, Lucy said, "It's about looking at my situation and what can I do to make myself a better person to be around other people" (personal communication, October 8, 2018). For Lucy, self-reflection in the clinical setting is sensing the outer world and acknowledging behaviors that may benefit someone other than herself.

Reviewing experiences to learn and move on. Melissa said self-reflection is "an overview of what happened during the day, where I could have done better or where I did well. Then I move on to learn better for the next time. Self-reflection is thinking about it, I don't analyze it" (personal communication, October 28, 2018). Melissa described a type of selfreflection that helps her think about past actions and to acknowledge how well she performed in the day. Melissa added reflective practice helps her let go of past experiences to learn how to improve her practice. Lastly, Melissa described self-reflection as viewing an experience in her mind without having to analyze every detail it may contain. 
Interaction leads to empathy, compassion, and understanding. Emily defined selfreflection as "being able to put myself in somebody else's shoes and see how I would feel in their shoes. It helps me be more compassionate and understanding” (personal communication, September 27, 2018). Emily believed self-reflective practices are used in the clinical setting to empathize with other people and to learn how similar circumstances would affect her own wellbeing. Emily learned how to understand the experiences of others by empathizing with them and feeling compassion for one's self. Beth described self-reflection in accordance with Emily, as a process that begins by interacting with others in the clinical setting. Beth said, "I interact; then I assess how it went, good or bad. Then I navigate so I have a positive outcome, and I understand the other person, they understand me too. That is self-reflection" (personal communication, December 4, 2018). When Beth interacted with others, she recognized reflective practice as a process to evaluate the positive or negative features of an experience. Despite the nature of an experience, Beth described reflective practices in terms of helping people relate to each other in a productive manner.

Experienced pediatric oncology nurses define self-reflective practices as a process of thinking about specific feelings, thoughts, emotions and behaviors that are specific to nursing care such as interaction, assessment, empathy, and compassion. When nurses interact with others, self-reflection is described as being helpful for achieving mutual understanding. In summary, participants defined self-reflection using their own perspectives but also shared similarities. Participants described self-reflection as being aware of specific moments or situations where they were able to pause, think, and process their feelings to improve their practice. For them, self-reflection was used not only to review a specific event but could also be considered a process to review the entire day. In conjunction with providing the definitions of 
self-reflective practice, participants in this study explained the advantages to using selfreflection.

\section{Advantages of Using Self-Reflection}

To Emily, the use of self-reflective practices is advantageous. Emily stated:

The advantage is it helps to relax me. Just like we tell our patients, it's like they're going to treatment and maybe it's something stressful or painful to them. When we see them stressed up and tensed up we tell them to take a deep breath and they do and they immediately start to relax ... I know the same thing works for me. (personal communication, September 27, 2018)

For Beth:

A self-reflective practice would be the way you take care of yourself to keep yourself positive, like self-care. Self-reflection is being able to look at yourself and after you've interacted with someone, did you help them? Did you make it worse? Do you feel like you reached them? How was self-reflecting with patients and how could you do it better? (personal communication, September 28, 2018)

Emily described self-care as "eating well, taking your lunch and actually eating and resting" (personal communication, September 27, 2018). To Melissa, the advantage of using selfreflection for self-care varied according to how nurses used self-reflection during their early years as novice nurses:

It really depends on the nurse per se because novice nurses are sometimes stuck in that learning mode and just trying to survive the shift and make sure they've done everything. They don't realize half the stuff they're doing. That comes after you're one to two years of getting comfortable in your shift like, yes, I can do this shift. I can take care of these 
patients. Then yeah, you need to be self-reflective on how you take care of yourself in this situation now. (personal communication, August 6, 2018)

Nicole described the advantage of using self-reflection as a process that protects and supports her decision to remain connected to the unit:

It's what I need to do to protect, to take care of me. That's what I feel. I love this unit. I can't imagine myself working on a different unit. I want to be here. I want to continue here. It's something I need to do in order to say, hey, I'm still okay. I can still continue ... what that means to me is stepping back, looking at a situation or an event, and reviewing what happened. Reviewing and acknowledging, okay, what did I feel from that? How did I feel that went? Was there something I could have done different? Or no, this went really well, and I'm proud of myself for that. That took a long time to get there. I have to be able to not be criticizing, but also encouraging and uplifting to myself. (personal communication, November 30, 2018)

Participants described using self-reflection in the clinical setting as advantageous to nurses by reminding them to breathe and relax, helping them to acknowledge self-care, and looking at their feelings and actions to develop better ways of helping patients. Once nurses gain practical knowledge during their early years, they recognize reflective practices are methods of self-care, which help them cope with the stressors of the workplace environment.

Self-reflection is used to cope with workplace stressors. Melissa used self-reflection to learn not to take things so seriously, "We learn from our past experiences. So, yeah, it's affected me in a way that I don't take everything so seriously. I'm more easygoing now with that" (personal communication, August 6, 2018). Melissa learned self-reflective practices alleviated 
harmful feelings. Similarly, through self-reflection, Beth is now able to separate or release emotions after providing care to her patients:

I've learned through the years how because you have to be able to detach a little bit. If you get too sucked into it, you can get really subjective and then you can't be a good nurse for that kid. (personal communication, September 28, 2018)

Pediatric oncology nurses who are too connected or emotional can feel overly involved in the personal lives of their patients and families. On finding various ways to cope, Beth said:

Through the years I find different ways to cope to help myself bounce back up like, what do I want to do with my life and what kind of life do I want to live? I want to make a difference in the world. (personal communication, September 28, 2018) Amanda described how reflecting and learning to cope in a productive manner helped her manage feelings:

I learned how to cope with my feelings about what was happening in a way that was productive and educational for me. So, that the next time, I have more ability to manage the scenario because I learned from my past experiences. (personal communication, October 19, 2018)

Amanda learned to use self-reflective practices by managing scenarios and understanding how those instances are beneficial for her professional development. Emily identified what makes her use self-reflection:

That's what makes me self-reflect. What am I feeling right now that I'm doing that and not even realizing it? I think it's a coping mechanism for me that I'm doing it subconsciously, not even realizing it until somebody says something. (personal communication, November 15, 2018) 
Emily reported how important it is to use self-reflection because, if she does not have the opportunity to reflect and separate herself mentally, feelings of stress build and become inundating. Emily stated, “I know when I don't self-reflect at the time when it's stressful at work, it impacts me because it impacts my mood or it becomes cumulative later and then it comes out" (personal communication, September 27, 2018). Nicole reported using self-reflection to acknowledge specific events to learn about them and move forward:

Self-reflecting, you use it to really separate yourself ... just acknowledge the events. Learn from it because, good or bad, there's always something we can learn from situations. Incorporate anything you can into your practice the next time you're there. It comes to a point where it's helped me to let it go. Before, when I couldn't let it go, I don't know, it eats up at you. It causes issues. You're like tense and you're angry and it's not okay. (personal communication, October 1, 2018)

In addition to using self-reflective practices to cope with workplace stressors, participants also stated that reflecting and being in tune with their feelings is beneficial for nurses.

Self-reflecting and being in tune. Being in tune with feelings of stress could benefit nurses. For example, Emily reported using self-reflection to acknowledge certain moments when she needed to be in tune with feelings:

There are times that we don't listen to our bodies or our minds when we're beginning to stress out. It can give out a certain body language or send a message out without even realizing it. If we can realize and be in tune to what we're feeling during stressful situations and be able to take that big deep breath, a cleansing breath to regroup, and be able to look at a situation, at different angles, and through somebody else's eyes, we are 
able to better serve a patient and help them out. (personal communication, December 4, 2018)

Beth had used self-reflection to acknowledge moments when positive energy proved helpful: When you have a nurse that's positive, hopeful, and has energy, you share with your patients. That helps them to have hope during the really stressful time in their life. A big part of fighting cancer is the mental component. Having nurses that are more in tune with how they feel, they're going to be more effective at helping people with their emotions .... I have to be in tune with my own emotions, and I think I'm reflecting on how it went, positive or negative. Then I have to go back and readjust to try and make it be positive. That's what it means .... I'm always talking about emotions and feelings, and asking myself how I feel as I become more in tune with my emotions. The more I do it; it's like a reflex. (personal communication, December 4, 2018)

The more Emily and Beth used self-reflective practice, the more it became a natural response whenever they felt out of balance with their feelings. Self-reflective practices appear beneficial to nurses because participants described the importance of processing feelings in a positive manner as essential to providing cancer patients with competent caregivers.

Self-reflection helps nurses address feelings of burnout. As the nurses in this study used self-reflection more, they developed coping methods to combat the harmful effects of burnout. Beth described her use:

I'm being aware of my emotions and my feelings. But then the more aware I am, it helps me cope better. If I stuff all my emotions and I don't talk about it, after a while it's going to start draining me physically. I might not understand what's going on, but I get this heaviness in my heart. When I'm processing everything and reflecting how I feel and 
taking care of myself along the way, then I'm not going to hopefully burnout. (personal communication, December 4, 2018)

Emily described being aware of her feelings of burnout and stated:

When I'm able to recognize that I'm becoming burnt out or something needs to change and I'm not wanting to get up because I'm stressed out to go to work, I start thinking of different things to do. That's when I start thinking, okay who am I? I'm not just a nurse. I'm not just a mom, and I'm not just a grandma or wife. What do I like to do? I have to start looking at what I'm doing, what I'm not doing and start finding the things that I need to do, or what I like to do, that makes me happy to counteract that stress. (personal communication, September 27, 2018)

Emily understood the benefits of using self-reflection as a tool that helped her feel fulfilled and happier at work and in her home life. Emily stated, "I self-reflect to see how I'm carrying myself, where I'm at in my burnout, and I start recognizing where my burnout is now" (personal communication, September 27, 2018). To Emily, burnout is a feeling, "When I do not want to come in to work" (personal communication, September 27, 2018). Furthermore, using selfreflection is an essential tool that helps Emily cope with emotions and return to work. Emily stated, "There's other ways to be able to cope with stress. I don't know if it's effective or not without being self-reflective" (personal communication, November 15, 2018). For the nurses, being self-reflective was beneficial for coping with feelings of stress and burnout. Participants stated that they often worked together and shared similar experiences to build camaraderie as a way to combat burnout. 
Self-reflection and a link between coping and camaraderie. I found using selfreflective practices to cope appears beneficial to experienced nurses. Over the years, Melissa learned how to distinguish coping from other forms of actions:

As I was a new nurse I would think, why are they laughing when the patient just died? But they [colleagues] are laughing with the family and everything. Then talking to them later, realizing it's how you're coping with the whole situation. (personal communication, August 16, 2018)

By talking with her colleagues and engaging in her own version of self-reflective practice, Melissa understood how reflecting with colleagues can be helpful in learning how to cope in the clinical setting. Emily had similar experiences with her colleagues. When referring to using selfreflective practices with her colleagues, Emily stated:

It helps camaraderie because that's when we share. After you self-reflect, we're able to share each other's experience or our coping methods and listen to other people. We can relate better. It's intimate. It creates bonds to be able to have camaraderie. One of the things that I know, in the years that I've worked, it doesn't matter how busy it is on the unit, if you have camaraderie, we can be okay. (personal communication, November 15, 2018)

Experienced pediatric oncology nurses in this study reported self-reflective practices are beneficial and enable nurses to develop healthful coping habits. Amanda described a type of clinical-based reflective practice with her colleagues that requires a nurse to be willing to share one's perception:

You can practice reflection individually, but in a group, in [the pediatric hematology/oncology unit], we also have a shared reflective practice that we do together. 
You have to be willing to share your perception. You can't do it if you're not going to share your perception. (personal communication, October 19, 2018)

Nurses described self-reflective practices as helping them cope with workplace stressors. Coping with workplace stressors includes being in tune with feelings and emotions. For example, nurses address feelings of burnout by using reflective practices individually or with their colleagues. Shared reflective practice help nurses develop camaraderie with colleagues, but nurses must be willing to share their perceptions with others to develop knowledge and understanding of oneself in the clinical setting.

Reflection helps nurses understand the self in relation to others. The conscious act of using reflection can enter the mind of a nurse through visual perception. When a nurse feels distress or sees another person suffering, reflective practice helps to form questions that identify possible solutions. Emily was able to recognize distress in a fellow colleague and used a clinicalbased reflective practice to learn how she could provide support:

This nurse, I could see it on her face how distressed she was. That's another thing, I'll look around and when I see a nurse like that, I can see that she says I haven't charted a thing and more than half the day's gone by. I haven't charted a thing. It's like, okay, what can I do to help you? So it's recognizing that and talking with them, what can I do? Sometimes they're so stressed out because they haven't had time to self-reflect, they're just trying to get through. Time's going by and they're getting more behind. (personal communication, November 15, 2018)

Emily was able to acknowledge distress in another individual because when she experienced comparable emotions, she used self-reflection. Emily described the value of using self-reflection in the clinical setting as integral to being a quality nurse: 
I don't see how I can nurse people without being self-reflective because it's such an intimate interaction. It's really important to reexamine one's self; see how I'm feeling in a situation. Whether it's the situation or how I'm feeling that day because it comes across to the patient. I feel like if I'm rushed, if I'm stressed, if I'm anxious, I give that off to the patient and their families. For that reason, it's really important to self-reflect. (personal communication, December 4, 2018)

It is essential for nurses to be aware of their feelings. The way nurses feel can be visible to patients and families. Nurses learn to define positive emotions and behaviors when they are aware of using self-reflection. Emily reported, "Self-reflective practices is stopping to take time out to examine one's feelings and actions towards situations and learning from them" (personal communication, December 4, 2018). Emily described her experience of using clinical-based reflective practice as being aware of the environment, taking time to recognize feelings of distress in oneself or another, and asking oneself the question, "Okay, what can I do to help you?"

For Amanda, the experience of using self-reflection in the clinical setting meant being a nursing professional as opposed to an employee who does work:

As a professional, it's part of who you are. If I were just doing a job, to me that's the difference between being a professional and doing a job. Doing a job, I don't have to embrace it. I can do it. (personal communication, October 19, 2018)

Similar to Amanda, Emily (personal communication, November 15, 2018) stated she did not believe nurses can be effective caregivers without using self-reflection. Emily described she believed nurses are task-oriented: 
It can be effective as far as getting tasks done. But nursing is not tasks ... but it has to be done after work hours because it's too busy during work. It's just choppy ... just finding a place, it can be a disadvantage in that sometimes I can't always find a place to do it or have enough time to be able to discuss it fully; phone ringing and people coming in so it's hard to do ... because of [the Health Insurance Portability and Accountability Act] I have to be careful where I talk and what I talk about. It's not always so easy because I have to be mindful of the content and finding a place where I can talk privately, which is not always possible. (personal communication, November 15, 2018)

Emily and Amanda believe nurses may not have the time or desire to use self-reflection because the stress nurses encounter in the workplace may have a negative impact on them or the obligations nurses have to their patients are guided by feelings of doing a job rather than being a skilled professional.

Experienced nurses can authentically share their feelings while providing care; however, according to participants, it is the reflective practice that can enhance care. Using self-reflection implies willingness for nurses to share a bit of themselves to learn and to grow. Amanda believed self-reflection is advantageous to nurses because "it's the willingness to bare your soul and the willingness to absorb, listen to, and really be open to another person's experience and learn from it" (personal communication, October 19, 2018). Amanda acknowledged the benefit of selfreflection as a process of understanding. To Amanda, self-reflection is not simply used to understand patients but also to help patients and their families understand nurses are caring professionals. Lucy echoed the words of Amanda and described the benefits of using selfreflection as being able to reach an understanding: 
I'm imparting all this information about self-reflectiveness, and I'm having to think, okay what's this? But then as I break down things one by one, I understand as we're going over this a second time what I want to get out of it. You're imparting that to me, and then I understand a bit more. So, that's the same thing as self-reflective, till we get to the point where we have an understanding. (personal communication, November 13, 2019) Lucy shared participating in an interview process on nurses and self-reflective practices was an opportunity to better understand what self-reflection means. To Emily, Lucy, and Amanda, the main benefit of using self-reflective practices was achieving understanding concerning the self and others. Melissa stated that nurses must learn to understand themselves and to communicate their feelings with supportive colleagues:

We tell all of our new nurses if you keep it in to yourself, you're doing yourself harm because most likely whoever you talk to will have experienced what you've experienced. You need to talk about your feelings and your emotions with people around you because most likely one of your coworkers that you're close to has already experienced that and knows how to get through it. It's normal; we will support you through that. The worst thing you can do is hold it in, hold it in and hold it in. Then you break and you may never become a [hematology/oncology] nurse anymore. You might leave the field completely and go do something completely different. (personal communication, September 2, 2018) Emily, Amanda, Lucy, and Melissa described how pediatric oncology nurses must use selfreflective practices to develop an understanding of the self and others and to be open to the possibility of sharing intimate feelings with trusted colleagues. To Melissa, it was paramount for nurses to communicate feelings with colleagues to thrive in their professional roles. 
I revealed many advantages of the use of self-reflective practices for nurses in this study, including to (a) develop methods of self-care, (b) develop coping methods to combat workplace stressors, (c) be in tune with their feelings, (d) address feelings of burnout, (e) develop camaraderie in the clinical setting, and (f) learn about who they are as professionals to better serve the specific needs of their patients. Experienced pediatric oncology nurses use selfreflective practices to learn from their experiences in the clinical setting and develop as specialized healthcare professionals. It is important to determine how nurses learn to use selfreflection to understand how and why they use it in the clinical setting.

\section{How Nurses Learned to Use Self-Reflective Practices}

I detail the experiences of nurses and how they acquired knowledge about using selfreflective practices in this section. Some nurses were exposed to self-reflection early in their nursing education. Other nurses learned about self-reflection from their clinical experience and by interacting with patients and their families.

Guided self-reflection enhances clinical reasoning. During nursing school, Beth had an instructor who left a lasting impact on how she used self-reflection to enhance clinical reasoning. Beth described how her instructor inspired her to share her feelings during difficult moments: "He's like, there's good and bad in everything, but it's how you perceive it that makes you be strong. That's what first got me interested in that. I always loved his class" (personal communication, September 28, 2018). In nursing school, Beth learned about clinical reasoning and how to assess her patients until a solution was established. For Beth, the process of assessing patients and reassessing their signs and symptoms was transformed through self-reflection:

When I started with nursing school, they're talking about the nursing process. I always have to assess the situation and then, what I'm going to plan to do? Then I have to 
reassess. I am always reassessing my interventions to evaluate how they are. Are they good or bad? If they're not working, then I have to reassess and try something different until I find something that works. (personal communication, December 4, 2018)

One method that helped Beth develop the ability to successfully assess patients during her clinical education was learning a guided approach to self-reflection:

We would write about our experience on the unit that day. What went well? What went bad? What did we find the most challenging? What were we most uplifted by? At the end of the semester, we had to turn our journal in. (personal communication, September 28, 2018)

During nursing school, Beth learned about self-reflective practices because she was required to participate in a structured approach, while other nurses learned about self-reflection from their clinical experience.

Reflecting and learning through interaction and relation. Beth learned how to use self-reflection by interacting with patients and family members. Self-reflection was an ongoing process by which Beth paid close attention to her own feelings and behaviors to better interact with patients:

It's a process of reflecting on how I interact with people like were my interactions helpful or not helpful? That's what self-reflective means like, looking back, how did I interact with the world? Was I effective in making it better? Or did I make the situation a little worse? That's what self-reflecting is, looking at my own behavior, my own thoughts and emotions and how I interacted with people. That's self-reflection. (personal communication, November 29, 2018) 
Like Beth, Amanda learned to use self-reflection by interacting with others, not necessarily by reflecting on every caring moment. Amanda shared, "As an experienced nurse, I reflect less on patient care and what I did in patient care, and I reflect more on how I interacted with all the players" (personal communication, October 19, 2018). Similarly, Lucy mused, "So, being selfreflective is: How is the best way for me to interact with the patients? To make sure I empathize?" (personal communication, November 13, 2018). The nurse-patient interaction was described as assisting nurses to use self-reflection and to learn and appreciate the experiences of others. For example, Emily learned to modify or adjust how she uses self-reflection to appreciate the experiences of another person:

I know it comes from pulling from my experiences, whether I realize it or not. Then at the same time, it's a combination of pulling from experiences but, yet, leaving it behind at times to be able to understand somebody else's experience. (personal communication, November 15, 2018)

The importance of learning self-reflection. One of the participants, Nicole, highlighted that self-reflection should be used both in nursing education and in the clinical setting. Nicole said self-reflection

should be required. If you are training individuals to be good nurses, to send out into the world, part of that in school is teaching them how to self-reflect, how to cope, how to come to terms or deal with. You're giving me the tools to think and how to read an [electrocardiogram]. You're giving me the tools to understand this medication; you're giving me those tools. But you're not once addressing, okay, what happens when things go right, when things go wrong, what happens? How do you then process that? How do 
you then deal with that? How do you put that into words, into actions, into whatever it takes to continue? (personal communication, October 1, 2018)

Nicole was adamant about the need to teach nurses how to use self-reflection in the clinical setting, describing it as an essential tool that can be highly useful for nurses. To her, selfreflection is a tool to help nurses learn how to process their emotions, similar to tools they use to perform their clinical tasks. Performing clinical tasks requires nurses to interact with patients and their families. For many participants, learning about self-reflective practices occurred during their nursing education and training and by interacting with others. Learning self-reflection to develop as care professionals and to use in the clinical setting helped nurses to understand the importance of making time for clinical-based reflective practice.

\section{Making Time for Clinical-Based Reflective Practice}

Experienced nurses make time to use self-reflective practices in the clinical setting.

Making time for self-reflection meant a lot to Emily because it helped her to continue working in the clinical environment. Emily stated, "Being self-reflective enables me to continue nursing. Not having that, there's no way I could be able to do this as long as I have. There's no way" (personal communication, September 27, 2018). Nicole and Lucy described how making time for self-reflection and relaxation helps them regain energy. Nicole stated:

When I have found myself in a situation that I'm extremely anxious and I'm overwhelmed because of my to do list or I have to do this presentation for work, I actually can step away. I will physically leave the unit. I will go outside. It might be for 2 minutes. It might be for 20 minutes. (personal communication, November 16, 2018)

Lucy said: 
I have to say to myself, you know what, take 20 minutes to clear your mind. I can't do anything. Patients are fine they're being covered. I know that. I just close my eyes. I have the TV on so I can't hear the door opening and closing. I relax. I get up, refreshed and ready to go again. (personal communication, October 8, 2018)

Nicole and Lucy described the experience of a clinical-based reflective practice as a moment that spans roughly two to 20 minutes. For Nicole and Lucy, it was possible for nurses to make time to rest and use self-reflective practices in the clinical setting. In Section 1, I described self-reflective practices to set the stage for learning about the unique experiences of nurses who use clinicalbased reflective practice. To better understand the actual experience of using self-reflective practices, participants were specifically asked to describe their individual experiences. In Section 2, I address how participants of this study recalled how using a clinical-based reflective practice appeared to their mind's awareness.

\section{Section 2: The Experience of Using Self-Reflective Practice in the Clinical Setting}

In Section 2, I discuss how each of the six participants described their unique experiences using self-reflection in the clinical setting and how participants creatively used abstract ideas to describe their experiences. For Lucy and Nicole, the experience of using self-reflective practice is like being at the epicenter of an experience. Lucy described the act of self-reflecting in the clinical setting like being at the center of a stack of mirrors:

I'm standing in the middle of two of them and I'm able to look through one mirror and it reflects back through the other mirrors. I'm looking, and that is reflecting off experiences that I've had. And then ... I'm actually in the center and in charge of my surroundings. (personal communication, November 24, 2018)

Nicole provided a vivid portrayal of her experience: 
It's really re-centering myself. I was on Google Maps and I think I opened it too far and it asked me, do you want to re-center. For some reason, that just clicked to me. To me, that is what this is. When I self-reflect, I'm making a very conscious effort to not just be a negative event at work. It could've been something that happened that was positive. Why can I also not be enriched or re-centered by that? I'm trying to find those opportunities where, okay, yes absolutely, I expect it with an unexpected event, with a negative event. But I also want to stop and take a moment when things are going really well, and just self-reflect on that. (personal communication, November 30, 2018)

Nicole described her experience as a struggle between using self-reflection after experiencing negative events or after positive ones. For Beth, the ability to use self-reflection was always present on the unit. Though not as apparent as other medical tasks, self-reflection can occur at any moment, especially, during moments of solitude. Beth stated:

It's automatic because when I walk away from someone, my mind starts going. It's like an emotional barometer, like how did that go? When I'm not talking with someone else, I'm walking down the hallway. I go walk to the supply room and I go draw up a medication. I have a moment by myself where I'm not interacting with someone. I'm thinking to myself .... It's when I walk out of a patient's room and I get a feeling when I'm with people. The energy in the room, is it positive, negative, tense, happy, joyful, sad, angry? There are all those emotional responses when I walk out of that room. I'm always asking myself, well, how do I feel? Then if it's something that's not so good, then what do I need to do here to make it better for myself, for the person, for my colleagues? (personal communication, December 4, 2018) 
Amanda shared a similar experience as Beth and described her experience in the clinical setting using visual perception. To Amanda, an experience on the unit can be viewed as colorless until she reflects and adds her own mixture of details:

If there aren't a lot of tasks that need to be done and there's more time, then I would take a little bit more time to self-reflect. At that point, I'm self-reflecting not only about the current scenario, but I'm incorporating all of these past experiences and how that might feed into how I handle this current scenario ... self-reflection is where I take the black and white framework and I put my color on it, and I say, to me, this looked this way. To me, this part looked this way. It's the personal component of it that makes it selfreflection. (personal communication, October 19, 2018)

Although participants were able to reveal the unique characteristics of their experiences using self-reflective practices, the nature of the clinical environment posed a challenge to experienced nurses when they desired to use a nurse-centric (i.e., nurse only) reflective practice.

\section{Challenges to Using Clinical-Based Reflective Practice}

Melissa said nurses have time only to acknowledge their feelings but cannot often process their feelings adequately or in-depth. She shared:

Some shifts you don't have time to reflect. You're chasing the whole day; you're running all day long, you don't have the time to reflect. Those rare occasions that it's a slow day, the only thing I could think about is that it brings back other experiences you've done and you talk about it. But, self-reflection during the day doesn't happen much for me. It doesn't, really—There's always something to do. So to turn my brain off, to relax and self-reflect, yeah, I don't think I could do that. (personal communication, August 6, 2018) 
Melissa described self-reflection as a process that requires the mind to shut off and relax, but without the time to relax, self-reflection cannot be fully accomplished. Emily went a step further and specifically described the challenges nurses face when trying to self-reflect in clinical settings. Emily described how nurses value using self-reflection during their shifts but rarely found adequate time to fully process emotions:

As it is right now when we double-check chemo, two nurses have to check chemo. We're not supposed to be interrupted because that's a really crucial thing. We have to make sure all the calculations are correct. It's hard to even get through a chemo check without the phone ringing or an interruption of any kind like a nurse asking questions and coming in. Or you need to re-evaluate because they need to get medication. It's hard to find time. (personal communication, September 27, 2018)

According to participants in this study, nurses need adequate time to effectively use selfreflective practices. Without adequate time for self-reflection, Emily described feeling there was always something else she could be doing for patients. Although self-reflection did occur in the clinical setting, Emily described needing more time during her shift:

We're supposed to get 15 minutes before lunch, sometimes 15 after. We never ever, ever, take those two 15-minute breaks. We just don't do it. The whole time I've been there, I've never done that. I've been guilty of that. Some units are able to do that. They've come to help us out. Then they'll call and say, I want to take my 15-minute break. And I'm looking at them and feeling like, we don't take 15-minute breaks. I've been guilty, and I know it, because I started thinking about it one day ... there's times I just have to take a deep breath to help calm down, relax, not be so stressed out and then keep on going. I take a deep breath because it's easy getting caught up and you really don't 
breathe. You're just so locked into a stressful situation. I have to remember to go and relax. Relax just take a deep breath, relieve some of that stress and let it out. (personal communication, November 15, 2018)

Emily described how she perceives the value of taking "a deep breath" and being able to "calm down and relax" while working on the unit. Emily believed that nurses need adequate time on the unit to rest and use self-reflection. Beth acknowledged the challenges to using self-reflection in the clinical setting and described a process of ongoing reflection as a possible solution:

I'm always reflecting on how it went all the time. When a lot is going on, like a lot of people need me, or maybe a patient is crashing and they're a full code still. I have to move fast and think fast and hustle, call the doctor. I don't have a lot of time to really go deep into myself, like for the emotional part of it. Or I'm being more objective and trying to get my task done to keep that patient alive. (personal communication, December 4, 2018)

In some cases, not having time for self-reflection may translate into not wanting to delve into the emotional aspects related to the process. For example, Nicole believed that some nurses might not want to share their feelings in the clinical setting. Nicole stated, "I also know nurses that don't want to have to do that when they're there ... because they feel they're going to fall apart and won't be able to recompose and continue" (personal communication, October 1, 2018). Nicole described other challenges nurses experience, such as emotionally and mentally falling apart:

Emotionally and mentally - It's hard to do it during the shift because what if I break down and I can't recompose .... It's not always easy. As a nurse, you don't want to feel like, oops, I messed up. Something happened and that affected my patient. Sometimes 
self-reflection is scary because of that .... The disadvantages are that sometimes I might find something that I don't like. I don't want to be the person to have messed up, made a mistake, made an error; I don't want to have to be the one like, oops, I was responsible, I dropped the ball, and I did this. (personal communication, October 1, 2018)

Beth also described the difficulties of using self-reflection in the clinical setting:

I have to shut down and take a step back. There's a moment and all of sudden something takes over. I don't know how to describe that but I get this clarity .... It's when there's the weight of the world on my shoulders. I can feel it at the end of the shift. I'm like, oh I feel drained. Then I have to find a way to vent, and then fill my energy level back up so I can come back and do it again the next day, or a week later whenever I have to come back .... I have to process all those emotions. I mean I can tell when I feel this heaviness in my heart, or on my shoulders. I'm like, okay, what's going on? Then I have to let myself feel sad or cry. I have to let it go and then move on. It's a new day the next day .... Sometimes I'm at a loss for words. Inside, I'm reflecting like, what can I say in this situation? It's really sad right now .... Maybe the challenge or disadvantage is I'm always wondering what my families need or are going through. It does take a lot out of me. (personal communication, November 29, 2018)

Both Nicole and Beth agreed that using self-reflective practices could sometimes make nurses realize feelings that could be more harmful than beneficial during the shifts. Using a nursecentric reflective practice may be best after the shift has ended and when nurses move away from the hospital setting. After describing the challenges of using a nurse-centric reflective practice in the clinical setting, the nurses revealed something unexpected in the data. When experienced 
nurses described their experiences using self-reflective practices in-depth, they find themselves often reflecting outside of the clinical setting, as described in the next section.

\section{Section 3: The Experience of Using Self-Reflective Practice Away From the}

\section{Clinical Setting}

Experienced nurses in this study described how using self-reflection to cope with stress and burnout occurs less in the clinical setting and more when they experience solitude or when they are driving home from work. Participants reported the privacy of an office or being in one's vehicle as an ideal time and place for genuine self-reflection. Melissa remarked, "You have to find what works for you. We're all different personalities, and we all have different personal situations growing up that contribute to reflecting on how you cope with a situation" (personal communication, September 2, 2018). Despite the difficulties of using self-reflective practices in the clinical setting, experienced nurses use reflection in a way that works well for them. Five of the six participants mentioned the experience of a nurse-centric reflective practice that began as they walked to their vehicles, entered, and closed the doors. Being alone in a vehicle made the nurses feel safe enough to release their emotions and be true to expressing whatever feelings they suppressed during their shifts. The nurses in this study described the necessity of using selfreflective practices after their shifts ended because they had more time and desire to fully process their feelings and emotions in a safe environment. All nurses, except Amanda who lived approximately five minutes away from work, described an experience of using self-reflection in vehicles and during the drive home. Beth added:

Whenever I commute and I'm driving, my mind is thinking about things and how I can always improve it or make it better. No one else is there to distract me. I mean except the traffic on the road but I'm driving and I'm alone with my thoughts. That's where I do 
most of my self-reflecting .... When I'm in the car, I have more time to really stop and think about what happened. It's an uninterrupted 30 minutes of thought. So yeah, I can be deeper. (personal communication, November 29, 2018)

For Beth, driving home was a chance to truly self-reflect. Processing emotions that were normally hidden away in the clinical setting were exposed in self-reflection as nurses got in their vehicles and began to drive further away from the hospital. Lucy reported:

There will be times I remember we'd lose a patient and on that drive home .... Even though I might be stellar at work, you get in that car and those tears come down and I think, okay, I did the best I could. So reflective, the meaning of it is, it's helpful for me. It's a release, that venting, talking, that's one way of releasing it. (personal communication, October 8, 2018)

Emily described a similar experience:

When I drive home, I go over the day in my mind. That's when I'm able to rehash. That's when I find myself trying to figure out, what could have been different? What could I have done different? What can I do tomorrow when I go back? (personal communication, September 27, 2018)

Emily said driving home and being encapsulated in her vehicle is a time when she does most of her reflective thinking. Similarly, when Nicole does not have a chance to use self-reflective practices after experiencing the death of one of her patients, she has learned to use the time in her vehicle as a moment to reflect and process her feelings. Nicole stated, "If I can't, I'll do it on my drive. I love my drive in the car. It's probably the most therapeutic time of the day for me" (personal communication, November 16, 2018). Even the type of vehicles or individual routes 
they routinely take to get home give nurses the opportunity to be genuine. Melissa, who has 18 years of clinical experience, stated:

When you go from the hospital to home it's the true me, raw, no filter. Whatever I feel at that time is when it comes out or if it's crying or I'm just thinking about the day. Or if I'm so mad and angry that I hit my steering wheel. You just go. I have made it home and gone back in and drove around. I enjoy driving and drive more to finish my anger or frustration. Then, come back and usually go to bed and sleep. (personal communication, October 28, 2018)

Self-reflective practice that occurs away from the clinical setting appears helpful for experienced nurses to feel they are true versions of themselves. In conjunction with using self-reflective practices in the clinical setting, participants in this study described using self-reflective practices when they leave the medical environment and drive away. The essence of using self-reflective practices means learning about who you are as a nurse plus who you are as an individual.

Findings of this qualitative study indicate experienced pediatric oncology nurses with at least 10 or more years of bedside experience use self-reflection in the clinical setting to the extent possible given their busy work environment. Self-reflective practice enables nurses to learn about themselves by relating with others, by thinking about how to care for their patients, or by providing support for colleagues who are showing signs of distress. The main challenge experienced nurses face in the clinical setting is having limited time on the unit to fully engage in using a nurse-centric reflective practice. Experienced nurses described using a nurse-centric reflective practice during moments when they could process thoughts and feelings with adequate time. Additionally, participants appreciate their down time and value the moments when they are left alone with their reflective thoughts. Authentically using a self-reflective practice is a 
phenomenon that nurses experience to a greater extent when they are away from the clinical setting. During moments of solitude, experienced pediatric oncology nurses reflect less on their professional roles and more on their abilities to relate with patients, families, and themselves. Experienced pediatric oncology nurses in this study described their self-reflective practices as a continuous process that moves in a medium between the clinical setting and one's private life. I discuss these findings in further detail in Chapter 5. 


\section{CHAPTER FIVE - DISCUSSION}

My aim for this study was achieved through the analysis of the interview transcripts, which the experienced nurses shared. The influence of self-reflection on each participant's wellbeing was described. Using self-reflection to process and manage the emotional demands of the workplace occurred when participants were reflecting with other colleagues, when they were away from the clinical environment (e.g., being in a private office), or during the drive home from work. Being reflective with colleagues or reflecting away from the clinical environment helped participants to process their feelings candidly and with enough time. I explored these findings using an inductive approach to understanding how expert-level nurses with 10 or more years of experience described using self-reflective practices in the clinical setting.

In this chapter, I discuss the meaning of the results and embed the findings in the context of the relevant literature. Moustakas (1994) described how developing a "summary section of transcendental phenomenological research" (p. 156) is important and necessary to effectively disseminate findings of a study and provide some opportunities for transferability. To provide a summary of this phenomenological investigation, I arranged Chapter 5 in three sections. In Section 1, I discuss a summary of the entire research project. I provide a detailed discussion of the phenomenon of using self-reflective practices according to the main objectives of this qualitative investigation in Section 2. Additionally, I compare the results of this study to previous research literature. In Section 3, I share information pertaining to (a) the implications for nursing education, nursing practice, and nursing leadership; (b) the significance and transferability of the study; (c) directions for future research; and (d) the limitations of the study, which will be followed by (e) the conclusion of the chapter. I begin the following section with a brief summary of the previous chapters. 


\section{Section 1: Summary of Research Project}

I developed this study to fill the gap in the literature pertaining to the experiences of expert-level nurses who use self-reflective practices in the clinical setting. Learning and understanding how these expert-level nurses describe the experiences of using self-reflection in patient care may shed light on how they use it to manage the emotional and physical demands of their profession. In Section 1, information from Chapters 1 through 4 are discussed and summarized. A summary of Chapter 1 and the factors that motivated me to develop this study are discussed next. I will discuss the main findings of this study in Section 2.

\section{Chapter 1}

In Chapter 1, I explained that experienced pediatric oncology nurses with 10 or more years of bedside experience demonstrated a propensity to effectively manage workplace stressors such as grief, moral distress, and burnout by using an array of coping methods when compared to new nurses (Benner, 1982, 2001; Boyle \& Bush, 2018; Conte, 2011; Enskär, 2012; Hinds et al., 1994). Self-reflection is one method that nurses have described using to combat the harmful effects of stress and burnout. I discussed how self-reflective practice is used by nursing educators, pediatric oncology nurses, and nursing students to (a) learn from one's experiences (e.g., self-reflection) and the experiences of others (e.g., group reflection), (b) develop professional practice and clinical reasoning, and (c) cope with feelings and emotions in a positive manner (Altounji et al., 2012; Caldwell \& Grobbel, 2013; Charalambous \& Kaite, 2013; Enskär, 2012; Hines \& Gaughan, 2014; Jestico \& Finlay, 2017; Linder, 2009; Mirlashari et al., 2017). Little is known, however, about how expert-level pediatric oncology nurses describe the experiences of using self-reflective practices in the clinical setting. 
Research question and purpose of the study. Chapter 1 was specifically developed to explain how pediatric oncology nurses use self-reflective practices to learn from experience and effectively cope with workplace stressors. I concluded Chapter 1 with a statement addressing the need to understand the experiences of using self-reflection from expert-level nurses. The guiding research question was: What is the lived experience of expert-level pediatric oncology nurses using self-reflective practices in patient care? My purpose of the study was to explore the experiences of pediatric oncology nurses with 10 or more years of bedside experience who use self-reflective practices in the clinical setting.

\section{Chapter 2}

In Chapter 2, I researched concepts, theories, and models that provided a framework for understanding the nature of self-reflective practices and how it relates to the context of nursing education and clinical practice. I described the theoretical underpinnings of reflective practice in nursing and carefully reviewed journal articles to explore how nurses learn from their personal and professional experiences by using reflective practice. My extensive review of the relevant literature did not find an adequate description of the meaning of the use of self-reflective practices as a method that helps pediatric oncology nurses develop self-care in the clinical setting. Therefore, I reviewed conceptual and applied journal articles to learn more about the meaning of using self-reflective practices as a method of self-care. In Chapter 2, I described various definitions and methods for understanding self-reflective practices in nursing education and its role in professional and personal development.

What is self-reflection? Self-reflection was defined in the literature as (a) a process of asking oneself simple questions to better understand an experience (Borton, 1970), (b) a process of personal and professional development (Atkins \& Murphy, 1994; Mann et al., 2009; 
Oluwatoyin, 2015; Ruth-Sahd, 2003; Schön, 1983), (c) a meaningful cycle of thinking that can lead to self-awareness and self-understanding (Atkins \& Murphy, 1994; Boud et al., 1985; Boyd \& Fales, 1983; Chacko \& Sreerenjini, 2012; Fowler, 2014; Gibbs, 1988; Kerfoot, 1996; Kolb, 1984; Pool, 2018; Smyth, 1989), and (d) a process of thinking that leads to action (Boud et al., 1985; Boyd \& Fales, 1983; Gibbs, 1988; Hentz \& Lauterbach, 2005; Kolb, 1984; Schön, 1983, Smyth, 1989). In Chapter 2, I also discussed how self-reflection could be used as a strategy to develop authentic and emotionally intelligent leaders (Akerjordet \& Severinsson, 2008; Avolio \& Gardner, 2005; Kerfoot, 1996; Shirley, 2006). In Chapter 2, I presented methods researchers have used to understand the nature of self-reflective practices and the lived experiences of nurses as opposed to the meaning of using self-reflective practices or how nurses develop knowledge of self-care by reflecting on their past experiences. Therefore, I reviewed how researchers explored the lived experiences of nursing professionals and self-care concepts in general. I discovered descriptive phenomenology and its unique methods would be advantageous for this endeavor, and I further described these in Chapter 2 (Charalambous \& Kaite, 2013; Hines \& Gaughan, 2014; Moustakas, 1994; Peden-McAlpine et al., 2005).

\section{Chapter 3}

I described the phenomenological research methodology and the specific methods I used in Chapter 3. I reviewed the history of descriptive phenomenology and its key concepts (Moustakas, 1994). The key concepts of descriptive phenomenology were described as (a) the natural and phenomenological attitudes (Christensen et al., 2017; Husserl, 1931; van Manen, 2014), (b) consciousness and intentionality (Christensen et al., 2017; Lopez \& Willis, 2004; Matua, 2015; Moustakas, 1994; Willis et al., 2016), (c) Epoché and bracketing, (d) the phenomenological reduction, and (e) the imaginative variation (Husserl, 1931; Lopez \& Willis, 
2004; Moustakas, 1994; van Manen, 2014). In Chapter 3, I discussed how descriptive phenomenology has been used as a qualitative methodology to understand a particular human experience by emphasizing the structure of consciousness known as intentionality (Moustakas, 1994; Linder, 2009). Intentionality was described as a process of the conscious mind where it visualizes particular objects and develops meaning from those specific experiences (Moustakas, 1994). In phenomenology, researchers explore the phenomena of human experience. For example, as the conscious mind continues to experience an object in space, it naturally develops greater layers of meaning. In order to learn something new about a person's experience, the researcher is required to refrain from viewing phenomenon in their perspective to learn and describe the perspectives of others (Linder, 2009; Moustakas, 1994).

Main features of descriptive phenomenology. Epoché, as described by Moustakas (1994), is a method researchers use to explore and learn something new (e.g., transcendental) about human experience. Epoché is a process where the researcher suspends judgments and biases (e.g., bracketing) throughout the entire research process (Moustakas, 1994). The researcher then returns to the phenomena by means of the phenomenological reduction with a renewed perspective and an open mind (Moustakas, 1994). I used the phenomenological interview as the main method for gathering information on human experience (Moustakas, 1994). Each participant completed three in-depth interviews. Upon completing the interviews with the six participants, the data were transcribed verbatim.

I then analyzed the transcripts (e.g., the textural descriptions of experience) using descriptive phenomenological methods as described by Moustakas (1994). The imaginative variation was specifically used after all steps of the data analysis were completed (Moustakas, 1994). The imaginative variation is a process where the researcher reflects on the overall 
experience and imagines possible ways those experiences manifest. I used the imaginative variation to develop a structural description of how expert-level nurses experienced using selfreflective practices (Moustakas, 1994). The final step of descriptive phenomenology is to form a textural-structural description of the essence of experience according to participants (Moustakas, 1994). Researchers explore and describe the essence of a particular human experience to form the basis for developing knowledge and understanding from human consciousness (Linder, 2009; Moustakas, 1994).

\section{Chapter 4}

In Chapter 4, results were arranged in three themes: (a) setting the stage, (b) the experience of using self-reflective practice in the clinical setting, and (c) the experience of using self-reflective practice away from the clinical setting. Using self-reflection in patient care was described as a thinking process that centers on interacting with patients, families, and colleagues. Additionally, self-reflection was described as a phenomenon that experienced nurses use to focus in on the emotional aspects of an experience. The goal of using self-reflection was to help nurses process feelings effectively and to reduce the harmful effects of workplace stressors. For example, participants in this study described making time to use self-reflection to learn about being more empathetic and compassionate because they valued the opportunities where they could relate with their patients better. When participants described using self-reflective practice for personal reasons (e.g., self-care), they described using self-reflective practice with other colleagues (e.g., group reflection) or when they were away from the clinical environment.

The essence of using self-reflective practices. In Chapter 4, the medical environment was described as a place where the conscious mind struggles to reflect on the whole nature of human experience. Participants of this study described how it takes too much time for nurses to 
reflect and pay attention to the totality of their experiences, which are a complex tapestry of feelings, emotions, thoughts, motives, needs, and desires. Experienced nurses have learned to use self-reflective practice when they encounter private spaces or when they were away from the medical environment, such as being in one's vehicle and driving. Being in one's vehicle is a private place where participants have been able to make time for self-reflection without much conflict and to fully attend to their feelings of stress and burnout in a productive manner. In the next section, I discuss the main findings and why participants used self-reflection in the clinical setting.

\section{Section 2: Discussion of the Findings}

My purpose for Section 2 is to provide a detailed discussion of the phenomenon of expert-level nurses and their experiences using self-reflective practices. In Section 2, I discuss the meaning of the results and how I compare the findings with the relevant literature. It is essential to point out that there were four studies identified in the literature specific to selfreflective practice and pediatric oncology nursing (Charalambous \& Kaite, 2013; Enskär, 2012; Hines \& Gaughan, 2014; Mirlashari et al., 2017). Therefore, I compare results in the next section using the larger body of the nursing literature, which I previously summarized in Chapters 1 and 2 of this study. A summary of Section 2 includes the main objectives along with a discussion of the most significant findings. The objectives of this study were (a) to explore experienced pediatric oncology nurses' descriptions of how they developed an understanding of reflective practices in patient care, (b) to explore their experiences of what self-reflective practices mean to them in the clinical setting, and (c) to explore their descriptions of using self-reflective practices and the potential impact this practice may have on their wellbeing and professional longevity. 
Findings of this study are arranged two ways: (a) why participants used self-reflective practices and (b) how participants described using self-reflective practices.

\section{Why Participants Used Self-Reflective Practices}

Findings of this current study indicated why experienced pediatric oncology nurses use self-reflection. The nurse-patient interaction was key to the experience of using self-reflective practices in the clinical setting. For example, reflecting on the nurse-patient interaction was essential for participants because it helped them attain empathy, compassion, and understanding for others. I find this to be consistent with a previous study that explored nurses' descriptions about being an expert nurse in pediatric oncology care (Enskär, 2012). In a study done by Enskär (2012), pediatric oncology nurses believed experts are confident in their knowledge, can often make decisions based on experience and intuition, and, thus, may spend more time using reflective practice to connect and empathize with patients and their families. Findings of my current study are similar to Enskär's (2012) findings, which indicated expert-level nurses reflect back on their patient care experiences with the purpose of establishing meaningful relationships in the future.

Self-reflection is intuitive. Interestingly, participants in my study described using selfreflection intuitively and with minimal conscious effort. In Enskär's (2012) study, participants reported expert-level pediatric oncology nurses use their experience and intuition to make decisions. Findings of this study provide knowledge that expert-level nurses indeed use selfreflection intuitively and as part of the decision-making process that cultivates positive relationships with pediatric oncology patients. Furthermore, experienced nurses make decisions that may benefit the wellbeing of their patients when using self-reflection intuitively. 
Using self-reflection cultivates positive relationships. Results of this study are consistent with findings of previous studies in which pediatric oncology nurses used selfreflection intuitively for the purpose of developing the art (e.g., presence) and science of their practice (Cantrell, 2007; Tanner, 2006). Additionally, nursing students who were learning in a similar setting described using self-reflection intuitively during the process of care (Gustafsson \& Fagerberg, 2004; Karimi et al., 2017; Rees, 2013). Tanner (2006), Morrison (2011), and Enskär (2012) agreed experienced pediatric oncology nurses knew what to do in the clinical setting and often used self-reflective practices as the embodiment of expert-level nursing. Although determining expert-level nursing status was not the main objective of this study, participants described using self-reflection in a manner that resembles expertise in pediatric oncology nursing. More research is needed to explore how using self-reflection intuitively can help nurses attain expertise. In the previous section, I discussed the central reason why participants used self-reflective practices (i.e., to cultivate positive relationships with patients and to develop empathy and compassion). I will discuss how experienced nurses described using self-reflective practices in patient care in the following section.

\section{How Participants Described Using Self-Reflective Practices}

I found pediatric oncology nurses with 10 or more years of bedside experience tend to use group reflective practices to cope with workplace stressors. Participants described using group reflection to discuss sensitive topics with colleagues (i.e., a patient's death or unsuccessful treatment regimens), which seemed to help build trust and camaraderie. These findings were consistent with O’Neill et al.'s (2019) study, and their exploration of liaison psychiatry nurses' experiences of attending psychology-led reflective practice groups. O’Neill et al. (2019) concluded a psychology-led group reflective practice can be useful for expressing 
commonalities, sharing emotions, and learning from those experiences in the context of liaison psychiatry nursing.

Group reflective practice and discussing difficult topics. Similar to O’Neill et al. (2019), participants in this study reported sharing feelings with others is difficult. A safe environment is required to effectively reflect and share feelings with peers. Group reflective practice can be effective "because it offers information from multiple sources and multiple perspectives” (Mann et al., 2009, p. 612). Pye (2013) reported the level of nursing experience may "have a positive impact" (p. 259) on how nurses collaborate to cope with workplace stressors. Caldwell and Grobbel (2013) theorized self-reflection can help nurses discuss difficult topics and understand their feelings in a productive manner. More research is needed to determine the impact of using group reflective practices as a method to develop coping among experienced pediatric oncology nurses.

Reflecting on emotionally negative and positive events. Surprisingly, participants described using individual reflective practices to process emotionally negative and positive events. Findings of this study seem consistent with previous studies, even when levels of experience differed between participants. In the studies of Charalambous and Kaite (2013) and Mirlashari et al. (2017), nursing students mostly used self-reflection after experiencing emotionally negative events during their clinical rotations in pediatric oncology. In Karimi et al.'s (2017) study, nursing students felt self-reflection was an effective method for minimizing negative emotions. Researchers have found similar findings on the calming effects of using individual reflective practices as a method for developing resilience (Jestico \& Finlay, 2017), self-care (Altounji et al., 2012), and coping (Enskär, 2012) among pediatric oncology nurses. 
It seems likely participants in this study shared similar experiences as the nursing students and nursing professionals described in previous studies. For example, using selfreflection after experiencing difficult events and learning its benefits over many years (e.g., managing workplace stressors for 10 or more years) may have inspired participants to reflect on both negative and positive events. More research is needed, however, to explore this phenomenon and the potential impact it may have on improving resilience, self-care, and coping in pediatric oncology nursing. Additionally, future researchers can focus solely on exploring the meaning of using self-reflection after pediatric oncology nurses experience positive patient events (e.g., full remission of cancer signs and symptoms) to determine its effect on coping with stress and burnout.

Using reflective practice effectively requires time. Participants described needing more time during their shifts to fully use reflective practice to effectively cope with their feelings. In addition, participants not only needed more time but also a private space in which they could be free to use authentic self-reflection. Results of this study are consistent with findings of Zander et al. (2010, 2013), Mirlashari et al. (2017), and Boyle and Bush (2018), who suggested using selfreflective practices in the clinical setting is often difficult and requires nurses to have adequate time. It remains difficult, however, to suggest how much time experienced pediatric oncology nurses need to effectively process harmful feelings of stress and burnout in a healthy manner, but it is clear that more time is desired.

Using self-reflection away from the clinical setting benefits nurses who desire more time to adequately address workplace stressors (Altounji et al., 2012). In a study done by Altounji et al. (2012), where the researchers developed a self-care retreat for pediatric oncology nurses, making time for self-refection away from the clinical setting was essential for pediatric oncology 
nurses to develop effective coping strategies and to address feelings of moral distress.

Conversely, in a study done by Moody et al., (2013) in which they explored how a mindfulnessbased course would be helpful for decreasing feelings of burnout in pediatric oncology nurses, it was found providing nurses with mindfulness training or opportunities for "reflecting on meaningful experiences and practice" (p. 276) can be taught in the workplace. Results of this study indicated that using self-reflection in multiple locations can be a productive experience, which helps pediatric oncology nurses cope with the demands of the workplace (e.g., stress, moral distress, and burnout). Making time for self-reflection (i.e., either in the clinical setting or away) may give experienced nurses the opportunity to slow down the pace of the medical environment or even minimize the harmful effects of working in a fast pace environment. Perhaps making time to use self-reflection in the clinical setting can lead to more precision and accuracy when dealing with complex treatment regimens fundamental to the pediatric oncology setting. In addition, making time to use self-reflection away from the clinical setting may help nurses process their feelings in a manner that is advantageous to their health and wellbeing.

Using self-reflective practices away from the clinical setting. Unexpectedly, the experienced nurses in this study described using self-reflective practice authentically when they were physically away from the clinical setting (e.g., a private office, taking a walk outside, or in their vehicles while driving home). Being away from the clinical setting and making time for reflection seem to benefit participants in managing their emotions in a productive manner. Consistent with previous studies (Altounji et al., 2012; Enskär, 2012), I found experienced nurses prefer to fully use reflective practices when they drive home from work. Being alone in one's car is a moment where experienced nurses have learned to fully engage in using selfreflection and to be fully attentive to the whole nature of their experiences. Interestingly, 
reflecting on the drive home was essential for participants to address a patient's death along with their feelings of grief, stress, and burnout.

Reflecting and managing workplace stressors. Although using self-reflection to process both negative and positive events were found to be essential in the clinical setting, driving home presented participants with a chance to be their true selves and reflect on their feelings of burnout. Most importantly, reflecting and being authentic during the drive home gave participants a sense of letting go of unwanted feelings of burnout so they could return to work the next day with a refreshed enthusiasm for their jobs as pediatric oncology nurses. In essence, having solitude (e.g., a private office), taking a brief walk outside, or being in the privacy of one's vehicle were useful for participants to use self-reflective practices and to manage their feelings of stress and burnout in a positive manner. Reflecting on the drive home and processing feelings of stress and burnout were unanticipated findings and require further exploration. Learning more about how pediatric oncology nurses describe their experiences of using selfreflection during their drive home from work may shed light how they develop coping habits that help them process feelings of stress and burnout. These results have implications for nursing students, educators, and leaders. In the following section, I will discuss the implications of these results in detail.

\section{Section 3: Implications and Significance}

The potential to understand why experienced pediatric oncology nurses use self-reflection and how they describe using it in patient care are the main implications of findings of this study. Further insight was also provided into how experienced nurses use group reflection in the clinical setting to develop authentic relationships with patients, which can improve a nurse's ability to empathize and have compassion. Furthermore, nurses who make time to use individual self- 
reflection and process their emotions during the drive home from work can potentially help reduce stress, moral distress, and burnout in a healthy and timely manner. Nursing education program managers, nurses, and nursing students may gain a greater understanding of experienced pediatric oncology nurses and their unique abilities to manage workplace stressors effectively from findings of this study. Lastly, this study was unique because I used transcendental phenomenological philosophy, methods, interviews, and data analysis to explore the experience of using self-reflection in patient care. In the final section of Chapter 5, I discuss specific implications of the findings, which include nursing education, nursing practice, and nursing leadership. Additionally, in Section 3, I discuss the significance and transferability of the study, along with the directions for future research and limitations of the study.

\section{Implications for Nursing Education}

Although this study produced modest information on the experience of using selfreflective practice in the clinical setting, I offer implications for nursing educators to consider. Working in the fast-paced environment of the pediatric oncology setting can reduce the time nurses have to focus on using self-reflection and self-care (Altounji et al., 2012; Caldwell \& Grobbel, 2013; Enskär, 2012; Hecktman, 2012). Furthermore, when pediatric oncology nurses do not have adequate time during their shifts to address their feelings of stress in a healthy manner (Barnard et al., 2006; Bowden et al., 2015; Chang et al., 2007; Davis, 2013; Emery, 1993; Emold et al., 2011; Gallagher \& Gormley, 2009; Hecktman, 2012; Kushnir et al., 1997; Morrison \& Morris, 2017), overwhelming stress may lead to nursing burnout or result in nurses leaving the profession (Barnard et al., 2006; Davis et al., 2013; Emold et al., 2011; Gallagher \& Gormley, 2009; Moody et al., 2013; Morrison \& Morris, 2017; Zadeh et al., 2012). To support the longevity of pediatric oncology nurses' careers and help them understand the potential benefits 
of making time for self-reflection in the clinical setting, nursing educators should begin to teach nursing students about group reflective practice.

Using group reflective practice might help experienced nurses discuss difficult topics with other nurses, which has the potential to build trust and camaraderie. Nursing students may experience difficulties when sharing their feelings with other students (Mann et al., 2009). Nursing educators can develop a pathway for deepening a reflective group culture through restorative practices (Schott Foundation, 2014). Researchers for the Schott Foundation (2014) stated, "Restorative practices are processes that proactively build healthy relationships and a sense of community to prevent and address conflict and wrongdoing" (p. 2). Educators can implement restorative practices, such as social-emotional learning (Schott Foundation, 2014), with students who are exposed to traumatic events.

Social-emotional learning in a group can teach students to reflect, recognize, and manage their feelings in a positive manner while cultivating a culture of trust and respect with their peers (Schott Foundation, 2014). The nursing educator can facilitate group reflective practice with students using a restorative practice framework for the purpose of developing mature students who can trust and collaborate well with others. In addition, nursing educators should continue to inspire students to develop research on group reflective practices and learn about the benefits of using them in the clinical setting.

Developing research with students could expose them to the importance of learning about reflection prior to entering the professional setting (Joyce-McCoach et al., 2013). Reflective practice can be taught and learned in the nursing context, however, only when deep reflective thinking is practiced (Mann et al., 2009). Mann et al. (2009) stated, "The factors that have been associated with its success appear to be a facilitating context, a safe atmosphere, mentorship and 
supervision, peer support and time to reflect" (p. 614). Understanding how nursing students can use deep reflective thinking has yet to be determined in the literature. Based on findings of this study, I suggest teaching students about group reflective practice and enabling them to share their inner thoughts with their peers can prove to be a useful strategy for learning about one's experiences in a thoughtful manner.

Nursing students who are looking to enter the pediatric oncology setting can be exposed to expert-level nursing skills by learning about the benefits of group reflection prior to entering the profession. Nursing students who have the capacity to reflect and share their emotionally charged feelings with other nurses can potentially build trusting relationships with their colleagues and decrease the likelihood of experiencing overwhelming stress and burnout. Chong (2009) and Rees (2013) purported teaching nursing students about reflection may help them process their emotions in a healthy manner as they gain more experiences in the clinical setting.

Educators who use reflection as a learning strategy could provide students with guidance, supervision, and a structured approach to ensure nursing students understand the benefits to their learning (Mann et al., 2009). Mann et al. (2009) concluded, "No educational strategy will provide a panacea for the challenges of professional practice; however, reflection may be a strategy, a 'habit of the mind' that can serve certain practitioners well, in certain situations" (p. 615). Educators can use results of this study to help nursing students understand that, when pediatric oncology nurses gain more years of experience, using group refection can prove useful for connecting with others and processing emotions in a healthy manner.

Lastly, using self-reflection should not solely be considered a method for professional development or strictly used in the context of the clinical setting (Bailey \& Graham, 2007; Chong, 2009; Rees, 2013). Indicated by findings of this study, experienced nurses make time to 
fully use reflective practices when they are away from the clinical setting and when they can be authentic with their feelings of stress and burnout. Nursing students can be inspired by educators to make time for self-reflection during moments of solitude or when they are away from the clinical setting as well. Nursing educators can teach students about how and when experienced nurses use self-reflection for their own wellbeing and how to apply those principles to their own lives and practice. For example, educators can develop assignments or provide resources for nursing students to understand where and when they may be best able to reflect and cope with their feelings of grief, stress, and burnout. When nursing students are given the opportunity to address their own feelings in a manner that is best suited for them, they can potentially reduce the likelihood of experiencing overwhelming feelings of stress and burnout. These opportunities can be developed so students can use reflection away from the clinical setting, in groups of nursing students and facilitators, or when they have moments of privacy during their day. What is ultimately essential in nursing education is not to force students to learn reflective practices, but to encourage them to find what works best for them and to continue developing and supporting those practices throughout their nursing careers (Mann et al., 2009).

\section{Implications for Nursing Practice}

Results of this study align with Enskär's (2012) finding that pediatric oncology nurses may perceive expert-level nurses as being confident in their clinical skills and may spend more time reflecting on experiences that cultivate positive relationships in the medical environment. Fully using self-reflective practices in the clinical setting, however, was not as helpful for pediatric oncology nurses to improve methods of self-care, like healing and recovering from the feeling of stress and burnout. Results of this study indicate experienced pediatric oncology nurses require adequate time and private spaces to fully use self-reflection to process emotions 
productively. Similar to Altounji et al.'s (2012) findings, I found it may be beneficial for pediatric oncology nurses to make time for reflective practice during private moments or when they are away from the clinical setting. More research must be conducted to understand how nurses with varying levels of experience can best be supported when they want to make time for using self-reflection in the clinical setting.

Results of this study add to the meaning of using reflective practices because the expertlevel nurses were aware of what would happen in the clinical environment if they did not use reflective practice effectively and with adequate time. These findings were similar to Enskär's (2012) findings. If the nurses have the capacity to know what happens when they do not use selfreflective practices, then they must also be aware of the benefits of using it. Self-reflection can be used as a strategy to find meaning in their roles as pediatric oncology nurses (Altounji et al., 2012; Enskär, 2012; Hines \& Gaughan, 2014; Oluwatoyin, 2015). Boyle and Bush (2018) stated, "Finding meaning in one's nursing practice is an effective strategy to offset negative affective corollaries" (p. 68). Boyle and Bush (2018) suggested "seminars, workshops, course series, and continuing educational offerings" (p. 69) can be developed to educate pediatric oncology nurses about the specific workplace stressors inherent in their profession and to help them find meaning in their lives. For example, information about using self-reflection in the clinical setting can be incorporated in conjunction with strategies such as patient debriefings.

Patient debriefings have been described as a "powerful tool used to deconstruct feelings of guilt, remorse, and sadness" (Boyle \& Bush, 2018, p. 156) after nurses experience a child's death. After a patient's death, support staff can modify debriefing sessions to incorporate some form of reflective practice that encourages nurses to find meaning in their practice and to effectively cope with their feelings of grief and sadness. Conducting more research combining 
both debriefing sessions and reflective practice can begin to shed light on how pediatric oncology nurses develop healthy coping habits in the clinical setting. Findings of this study fill the gap in the literature on expert-level nurses. Participants who identified with using selfreflective practices were able to reflect back on the health and wellbeing of their patients and learned to develop healthy coping habits during their shift, especially in private spaces or away from the clinical environment.

Hospital leaders and administrators may find value in these results. Hospital administrators who employ pediatric oncology nurses should promote healthy work environments and cultures where employees are able to choose the moments when they feel free to use self-reflection as needed. Motivating their employees and staff to use reflective practices naturally and during moments when reflection can be more beneficial than unfavorable can help all nurses develop the patience to value and perceive the true nature of the clinical setting (i.e., stressful, challenging, and rewarding). Experienced nurses are not oblivious to the demands of the pediatric oncology setting but they are susceptible to feeling of distress and feeling burnt out (Boyle \& Bush, 2018). Therefore, nurse managers may find value in findings of this study to develop recruiting methods to identify nurses who use self-reflective practices and support current employees who identify with using reflection in the clinical setting.

Cultivating the social and emotional health of pediatric oncology nurses is critical to the type of care they provide to their patients (Cantrell, 2007). A medical culture where nurses are not allowed to take breaks or where it is perceived to be socially unacceptable to do so is not a healthy practice in pediatric oncology nursing, or any other type of professional setting. According to participants of this study, using self-reflection as an ongoing process to learn and grow from past experiences can help mitigate harmful feelings of stress and burnout. 
Furthermore, using group reflective practices can theoretically help pediatric oncology nurses share their feelings and emotions with other colleagues whom have shared similar experiences (Altounji et al., 2012; Mann et al., 2009). Though further research is needed, I suggest using selfreflective practices individually or with others had a positive impact on developing self-care in the clinical setting. Therefore, hospital administrators can develop programs in which nurses are provided with adequate time and space to use self-reflective practices at their discretion and with supportive staff.

\section{Implications for Nursing Leadership}

Nursing leadership is responsible for developing programs and cultivating behaviors (e.g., authenticity, self-awareness, emotional intelligence, resilience) that include a safe and healthy environment for nurses (Akerjordet \& Severinsson, 2008; Avolio \& Gardner, 2005; Chacko \& Sreerenjini, 2012; Kerfoot, 1996; Pool, 2018; Shirley, 2006). Developing programs where nurses learn about self-reflective behaviors can potentially improve their capacity to cope with workplace stressors in a positive manner (Horton-Deutsch \& Sherwood, 2008). I found experienced nurses described self-reflective behaviors that potentially improve their emotional competence (e.g., group reflection in the clinical setting or individual reflection in private spaces or during the drive home). To espouse such behaviors, nursing leaders must figure out how to identify self-reflection in the clinical setting. The problem nursing leaders face is how to identify self-reflective behaviors from other forms of thinking (e.g., intuition, resilience, tacit knowledge). Studies have shown, however, self-reflection is not easily distinguishable in the clinical setting (Boyle \& Bush, 2018; Carlsson et al., 2002).

Previous researchers agree with the main findings of this study (Cantrell, 2007; Edwards, 2003; Fowler, 2014; Gustafsson \& Fagerberg, 2004; Karimi et al., 2017; Rees, 2013; Tanner, 
2006); self-reflection is often tacit in the clinical setting. Mann et al. (2009) identified similar findings in their systematic literature review on reflection and reflective practices in healthcare professional education. Nevertheless, experienced nurses use self-reflection intuitively when they interact with their patients. For example, participants described using self-reflection as method that is essential to the process of care, specifically to develop authentic relationships in the clinical setting. A possible solution for nursing leaders could be to develop mentorship programs to bring novice and expert-level nurses together.

Mentorship programs can be developed to help novice nurses shadow experienced nurses in the clinical setting. Shadowing expert-level nurses can potentially help novice nurses learn how to identify and understand self-reflective behaviors, which manifest during the nurse-patient interaction. In addition, experienced nurses described using group reflective practices to discuss difficult topics with other nurses. Mentorship programs that bring novice nurses together with expert-level nurses may shed light on the benefits of using group reflective practice in the clinical setting. Nursing leaders want pediatric oncology nurses to connect authentically and collaborate effectively during the process of care (Boyle \& Bush, 2018). Developing mentorship programs for novice nurses to learn how experienced nurses develop reflective behaviors can produce a coalition that strives to provide patients with quality care.

Lastly, participants in this study used self-reflective practices away from the clinical setting (i.e., private spaces) because it was perceived as an integral part of being a professional and a thinking process that helped them care for themselves as human beings (e.g., self-care). For instance, participants described making time to use self-reflection away from the clinical setting (e.g., during the drive home). Reflecting during the drive home from work gave participants an opportunity to process and let go of unwanted feelings of stress and burnout. 
Nursing leaders can develop programs away from the clinical setting (e.g., self-care retreats) to give nurses adequate time and space to effectively process their feelings of stress and burnout (Altounji et al., 2012). Nursing leaders can also develop programs that incorporate private spaces, in particular, one's vehicle.

Programs can be called "driving with an expert nurse," when an experienced nurse adopts a novice nurse for the day and takes them on a "reflective ride." Developing programs that connect expert and novice nurses together may produce nurses who learn the value of collaboration and understand the benefits of using group reflective practices away from the medical environment. Developing programs away from the clinical setting can help nursing leaders provide a safe and healthy alternative to using self-reflection in the clinical setting. Nursing leaders may find benefits from the evidence that expert-level nurses demonstrate reflective behaviors in and away from the clinical setting. The importance of this study was exploring the experiences of expert-level nurses who use self-reflection in patient care. In the following section, I discuss the significance and transferability of this study and its impact on understanding the experience of using self-reflection in pediatric oncology nursing context.

\section{Significance and Transferability of the Study}

This study was among the first of its kind to investigate how expert-level pediatric oncology nurses described their experiences using self-reflective practices in the context of the clinical setting. A momentary look into the minds of experienced nurses is found in this study, which may offer hospital administrators, nurse managers, educators, and practitioners an opportunity to understand what using self-reflection means to experienced pediatric oncology nurses. Nurses who identify with using self-reflection describe having the freedom, space, and time to see the entire complex field of human experience when they have moments to rest or 
when they find themselves away from the clinical setting. Our understanding of the nature of self-reflective practices, according to experienced pediatric oncology nurses, may be improved based on information from this study.

Transferability refers to how results of qualitative research can be generalized or transferred to other settings (Bailey, 2007). Using and describing specific recruiting (e.g., purposive sampling) methods may help readers understand the potential transferability of this qualitative research (Bailey, 2007). As I previously described in Chapter 3, participants were recruited using purposive and snowball sampling techniques. Researchers could use similar recruiting methods to obtain information that pertains to other nursing professionals and their experiences using self-reflective practices. Furthermore, Bailey (2007) stated, "The more details about a setting that the researcher provides, the more apt readers are to extrapolate the findings to other settings" (p. 183). To demonstrate transferability, thick description about the context and social setting of the participant's experiences using self-reflective practices were provided throughout the entire data collection process. In the future, researchers can use similar sampling techniques and provide a detailed description of the data collection process to enhance transferability of their data.

\section{Directions for Future Research}

Exploring different methodologies can be helpful for learning more about nurses and their experiences using self-reflection in the clinical setting. Furthermore, developing broader research questions can be helpful by identifying the value of using self-reflection in nursing and its potential impact on patient outcomes. Future researchers may also want to differentiate reflective nurses from nurses who do not use self-reflection as readily in the clinical setting. Comparing reflective nurses with non-reflective nurses can shed light on the meaning of using 
self-reflective practices from nurses employed in different hospital and in various types of nursing practice (Mann et al., 2009).

Researchers can use quantitative methods to measure the effects of using reflection on a nurse's physiology (e.g., heart rate, blood pressure, and body temperature) in relation to their perceived feelings of stress (e.g., mapping brain waves during reflective practice). Qualitative methods can then be used to explore their experiences of using individual or group reflective practice with their colleagues and with patients and their families. For example, to know if being reflective in a group can influence patient outcomes, researchers could begin to focus on developing ethical studies that determine how and if this is possible in the clinical setting. Future researchers can explore a variety of research questions, such as:

- Does individual or group reflective practice prove to be more important and beneficial in the pediatric oncology setting for improving patient outcomes?

- What happens in a group of pediatric oncology nurses when they use reflective practice in the clinical setting?

- Would group reflective practice be better for developing self-care when compared with self-reflection alone?

- Does using group reflective practice with other nurses cause more stress and burnout?

- Do nurses get more out of the reflective experience when they have adequate time and place to do so?

- Can nurses develop their reflective practices and methods of self-care when they interact with their patients and families?

- What are the effects of being reflective according to the nurse and their patient? 
- Does the reflective practice interaction have a direct effect on producing positive patient outcomes?

More research can be developed to compare the experiences of nurses who identify with using ongoing self-reflective practices with other nurses who describe using it only in a professional context. Researchers would be required to create specific survey tools (i.e., using theoretical concepts and models) that help nurses determine or identify if they possess reflective skills that specifically pertain to stress management in the nursing context to complete this type of study. By using an array of methodologies, future researchers can better understand the various uses and meanings of individual or group reflective practices and their subsequent effects on the health and wellbeing of patients and nursing professionals. Additionally, the opportunity for a nursing student to learn and understand if they can handle working in the pediatric oncology setting prior to their employment may minimize the risk of nurse burnout and maximize nurse retention. The paucity of information on reflective practice in the pediatric oncology setting makes it difficult for nursing students to identify specific characteristics of a reflective nurse practitioner.

Researchers must continue to use qualitative measures to explore the meaning of using reflective practices from a larger sample of the population and with a diverse group of nursing professionals and students. The goal of using reflective practice is to develop a keen awareness of the self in relation to others and to produce positive health outcomes for patients and nurses. Therefore, developing more research so researchers can predict when nurses require the most reflective practice during their daily lives can potentially increase the likelihood of nurses who feel healthy and capable of being fully present in the clinical setting. While being attentive to the nature of this phenomenological study (i.e., explore the lived experience of experience) and to 
the descriptions of each participant, some limitations of the study become apparent. Before concluding the presentation of this study, I provide the limitations.

\section{Limitations of the Study}

I took steps to minimize limitations at the beginning and throughout the research process. In this section, I discuss five specific limitations in an effort to provide recommendations on how to address them for future studies. The first limitation of this study was my review of the relevant literature did not provide sufficient evidence on expert-level pediatric oncology nurses with at least 10 or more years of bedside experience and their descriptions of using self-reflective practices in the clinical setting. Searching the nursing literature for nurses with specific experiences proved to be difficult.

A second limitation was all participants recruited were female. I developed sampling procedures for this study to obtain rich information from experienced nurses who could be identified as using self-reflective practices in the clinical setting. Male nurses were not excluded deliberately, rather, they were subjected to the snowball sampling procedures of the study. This meant other nurses were not able to identify and recommend male nurses who fit the inclusion criteria. For future studies, extending the inclusion criteria to include novice or expert male pediatric oncology nurses may be useful for examining their experiences using self-reflection and comparing them with their female counterparts.

A third limitation was the social desirability of this study. Van de Mortel (2008) said, "Socially desirable responding is the tendency for participants to present a favorable image of themselves.... Socially desirable responding is most likely to occur in responses to socially sensitive questions" (p. 41). In descriptive phenomenological research, the primary researcher must share their thoughts, feelings, and ideas that pertain to the development of this study 
(Moustakas, 1994). During the beginning and throughout the entire research process, I communicated with participants on a weekly basis on their scheduled interview dates or pertaining to their interview transcriptions. By communicating with each participant, they may have been well aware of my passion for developing this type of study. The social desirability of this study may have influenced how each participant responded to the interview questions during all phases of the interview process (van de Mortel, 2008).

A fourth limitation was using Husserl's TP to obtain in-depth information on the human experience (Moustakas, 1994). Developing a phenomenological research study was a difficult task considering the amount of information the primary researcher is required to learn prior to the start of the investigation. Husserl's phenomenology is based on developing an understating of the nature and structures of human consciousness (van Manen, 2014). To understand human consciousness from another's perspective requires specific methods. For example, the epoché or bracketing was the ability for the primary researcher to develop a rich description of his personal thoughts, opinions, biases, and presumptions about the phenomenon in question (Moustakas, 1994). I strived to develop a complete and detailed account of my own experience using selfreflective practices prior to the start of the investigation and throughout the entire research process.

In Husserl's (1931) phenomenology, the primary researcher is required to abstain from seeing the phenomenon through his own lens to see the experiences of another (Moustakas, 1994). I aimed to be open and truthful about my personal experiences with the phenomenon and to refrain from adding my personal biases and opinion during the interview process. The most challenging thing about using Husserl's phenomenology was that it did not allow for the interpretation of text, drawings, pictures, or any forms of communication or media that was not 
part of the experience of each participant. I admit it was very difficult to understand another person's experience without feeling the need to see it through my own lens. Nevertheless, I endeavored to be truthful and authentic to provide a credible and valid description of the experiences of nurses who use self-reflective practices in the clinical setting.

The fifth limitation was not pursuing experienced nurses who were ethnically or culturally diverse. Although some participants may identify with specific ethnicities and cultures, this study was not concerned with diversity of participants. The recruitment process was only based on years of experience in pediatric oncology nursing (i.e., 10 or more years) and whether nurses identified with using self-reflective practices in their daily lives. A limitation of this study may have been the lack of diversity of participants, which may have influenced how they described their experiences using self-reflective practices. Exploring diversity among participants may yield a greater understanding of the process of using self-reflective practices for the purposes of the patient and for the nurse (i.e., self-care).

\section{Conclusion}

I was dedicated to using phenomenological methods to explore pediatric oncology nurses' experiences who have 10 or more years of bedside experience and who identify with using self-reflective practices in the clinical setting in this qualitative study. The descriptive phenomenological approach provided an initial understanding of the nurses' lived experiences. Experienced female nurses described self-reflection in the pediatric oncology setting as taking a brief moment to pause and think about events specifically related to managing professional boundaries and developing meaningful relationships with others. Stopping to use self-reflection, however, was perceived as challenging. Participants described the importance of integrating selfreflection as best they could during their shifts but find the time to fully use it in private spaces 
and when they move away from the clinical setting (i.e., entering the safe and uninterrupted environment of their vehicle). Being away from the clinical setting and being alone with one's reflective thoughts was associated with a greater propensity for participants to cope with workplace stressors in a healthy manner.

When experienced nurses moved away from the clinical setting and made time for indepth self-reflection, they perceived this practice as a coping method. These results indicate using self-reflection as a coping method helped participants to process feelings (e.g., being overwhelmed, stressed, helpless, and having minimal energy during their shifts for self-care) in a healthy and timely manner. In essence, using self-reflective practices was described as a thinking process that flows between the professional and personal context, which helped experienced nurses to learn and develop empathy, compassion, self-awareness, and self-care. More research is needed to understand why nurses use self-reflection as a coping method when they are away from the clinical setting. Understanding the features of being reflective in private spaces can help future researchers and nursing educators understand the nature of developing healthy coping methods for nurses entering the pediatric oncology setting. 


\section{References}

Akerjordet, K., \& Severinsson, E. (2008). Emotionally intelligent nurse leadership: A literature review study. Journal of Nursing Management, 16, 565-577. doi:10.1111/j.13652834.2008.00893.x

Altounji, D., Morgan, H., Grover, M., Daldumyan, S., \& Secola, R. (2012). A self-care retreat for pediatric hematology oncology nurses. Journal of Pediatric Oncology Nursing, 30, 18-23. doi:10.1177/1043454212461951

Atkins, S., \& Murphy, K. (1993). Reflection: A review of the literature. Journal of Advanced Nursing, 18, 1188-1192. doi:10.1046/j.1365-2648.1993.18081188.x

Atkins, S., \& Murphy, K. (1994). Reflective practice. Nursing Standard, 8(39), 49-56. doi:10.7748/ns.8.39.48.s64

Avolio, B. J., \& Gardner, W. L. (2005). Authentic leadership development: Getting to the root of positive forms of leadership. The Leadership Quarterly, 16, 315-338. doi:10.1016/j.leaqua.2005.03.001

Bailey, C. A. (2007). A guide to qualitative field research (2nd ed.). Thousand Oaks, CA: Pine Forge.

Bailey, M. E., \& Graham, M. M. (2007). Introducing guided group reflective practice in an Irish palliative care unit. International Journal of Palliative Nursing, 13, 555-560. doi:10.12968/ijpn.2007.13.11.27591

Barbour, J. F. (2013). The making of a butterfly: Reflective practice in nursing education. International Journal of Human Caring, 17(3), 7-12. doi:10.20467/1091-5710.17.3.7 
Barnard, D., Street, A., \& Love, A. (2006). Relationships between stressors, work supports, and burnout among cancer nurses. Cancer Nursing, 29, 338-345. doi:10.1097/00002820200607000-00013

Beckstrand, R. L., Rawle, N. L., Callister, L., \& Mandleco, B. L. (2010). Pediatric nurses’ perceptions of obstacles and supportive behaviors in end-of-life care. American Journal of Critical Care, 19, 543-552. doi:10.4037/ajcc2009497

Benner, P. (1982). From novice to expert. American Journal of Nursing, 82, 402-407. doi: $10.2307 / 3462928$

Benner, P. (2001). From novice to expert: Excellence and power in clinical nursing practice. Englewood Cliffs, NJ: Prentice Hall.

Bevan, M. T. (2014). A method of phenomenological interviewing. Qualitative Health Research, 24, 136-144. doi:10.1177/1049732313519710

Borton, T. (1970). Reach, touch, and teach. New York, NY: McGraw-Hill.

Boud, D., Keogh, R., \& Walker, D. (1985). Reflection: Turning experience into learning. London, England: Kogan Page.

Bowden, M. J., Mukherjee, S., Williams, L. K., DeGraves, S., Jackson, M., \& McCarthy, M. C. (2015). Work-related stress and reward: An Australian study of multidisciplinary pediatric oncology healthcare providers. Psycho-Oncology, 24, 1432-1438. doi:10.1002/pon.3810

Boyd, E. M., \& Fales, A. M. (1983). Reflective learning: Key to learning from experience. Journal of Humanistic Psychology, 23, 99-117. doi:10.1177/0022167883232011 
Boyle, D. A., \& Bush, N. J. (2018). Reflections on the emotional hazards of pediatric oncology nursing: Four decades of perspectives and potential. Journal of Pediatric Nursing, 40(May-June), 63-73. doi:10.1016/j.pedn.2018.03.007

Bucholtz, M. (2000). The politics of transcription. Journal of Pragmatics, 32, 1439-1465. doi:10.1016/S0378-2166(99)00094-6

Burns, S., \& Bulman, C. (2000). Reflective practice in nursing: The growth of professional practitioner (2nd ed.). London, England: Blackwell Science.

Caldwell, L., \& Grobbel, C. C. (2013). The importance of reflective practice in nursing. International Journal of Caring Sciences, 6, 319-326. Retrieved from http://www.internationaljournalofcaringsciences.org

Cantrell, M. A. (2007). The art of pediatric oncology nursing practice. Journal of Pediatric Oncology Nursing, 24, 132-138. doi:10.1177/1043454206298842

Carel, H. (2011). Phenomenology and its application in medicine. Theoretical Medicine and Bioethics, 32, 33-46. doi:10.1007/s11017-010-9161-x

Carlsson, G., Drew, N., Dahlberg, K., \& Lützen, K. (2002). Uncovering tacit knowledge. Nursing Philosophy, 3, 144-151. doi:10.1046/j.1466-769X.2002.00092.x

Chacko, B., \& Sreerenjini, B. (2012). Reflective practice in nursing. International Journal of Nursing Education, 4(2), 43-45. Retrieved from http://www.ijone.org Chang, A., Kicis, J., \& Sangha, G. (2007). Effect of the clinical support nurse role on workrelated stress for nurses on an inpatient pediatric oncology unit. Journal of Pediatric Oncology Nursing, 24, 340-349. doi:10.1177/1043454207308065 
Charalambous, A., \& Kaite, C. (2013). Undergraduate nursing students caring for cancer patients: Hermeneutic phenomenological insights of their experiences. Health Service Research, 13(63), 1-13. doi:10.1186/1472-6963-13-63

Charalambous, A., Papadopoulos, R., \& Beadsmoore, A. (2008). Ricoeur's hermeneutic phenomenology: An implication for nursing research. Scandinavian Journal of Caring Sciences, 22, 637-642. doi:10.1111/j.1471-6712.2007.00566.x

Chong, M. C. (2009). Is reflective practice a useful task for student nurses? Asian Nursing Research, 3, 111-120. doi:10.1016/S1976-1317(09)60022-0

Christensen, M., Welch, A., \& Barr, J. (2017). Husserlian descriptive phenomenology: A review of intentionality, reduction, and the natural attitude. Journal of Nursing Education and Practice, 7(8), 113-118. doi:10.5430/jnep.v7n8p113

Conte, T. M. (2011). Pediatric oncology nurse and grief education: A telephone survey. Journal of Pediatric Oncology Nursing, 28, 93-99. doi:10.1177/1043454210377900

Conte, T. M. (2014). The lived experience of work-related loss and grief among pediatric oncology nurses. Journal of Hospice \& Palliative Nursing, 16, 40-46. doi:10.1097/NJH.0000000000000019

Creswell, J. (2014). Research design: Qualitative, quantitative, and mixed methods approaches (4th ed.). Thousand Oaks, CA: Sage.

Davis, S., Lind, B. K., \& Sorensen, C. (2013). A comparison of burnout among oncology nurses working in adult and pediatric inpatient and outpatient settings. Oncology Nursing Forum, 40, E303-E311. doi:10.1188/13.ONF.E303-E311 
DeFreese, J. D., \& Mihalik, J. P. (2016). Work-based social interactions, perceived stress, and workload incongruence as antecedents of athletic trainer burnout. Journal of Athletic Training, 51, 28-34. doi:10.4085/1062-6050-51.2.05

De Graves, S., \& Aranda, S. (2005). When a child cannot be cured-reflections of health professionals. European Journal of Cancer Care, 14, 132-140. doi:10.1111/j.13652354.2005.00520.x

Dolphin, S. (2013). How nursing students can be empowered by reflective practice. Mental Health Practice, 16(9), 20-23. doi:10.7748/mhp2013.06.16.9.20.e867

dos Santos, L. S. B., Costa, K. F. L., Leite, A. R., Leite, I. D. R., Sacramento, N. T., Oliveira, G., $\&$ dos Santos, G. (2017). Emotional perceptions and reactions of nursing professionals assisting children with cancer. Journal of Nursing, 11, 1616-1623. doi:10.5205/reuol.9763-85423-1-SM

Duke, S., \& Appleton, J. (2000). The use of reflection in a palliative care program: A qualitative study of the development of reflective skills over an academic year. Journal of Advanced Nursing, 32, 1557-1568. doi:10.1046/j.1365-2648.2000.01604.x

Dunn, K. S., Otten, C., \& Stephens, E. (2005). Nursing experience and the care of dying patients. Oncology Nursing Forum, 32, 97-104. doi:10.1188/05.ONF.97-104

Edmonds, C., Lockwood, G. M., Bezjak, A., \& Nyhof-Young, J. (2012). Alleviating emotional exhaustion in oncology nurses: An evaluation of Wellspring's "Care for the Professional Caregiver Program.” Journal of Cancer Education, 27, 27-36. doi:10.1007/s 13187-011-0278-z

Edwards, S. (2003). Critical thinking at the bedside: A practical perspective. British Journal of Nursing, 12, 1142-1149. doi:10.12968/bjon.2003.12.19.11799 
Emery, J. E. (1993). Perceived sources of stress among pediatric oncology nurses. Journal of Pediatric Oncology Nursing, 10(3), 87-92. doi:10.1177/104345429301000303

Emold, C., Schneider, N., Meller, I., \& Yagil, Y. (2011). Communication skills, working environment, and burnout among oncology nurses. European Journal of Oncology Nursing, 15, 358-363. doi:10.1016/j.ejon.2010.08.001

Enskär, K. (2012). Being an expert nurse in pediatric oncology care: Nurses' descriptions in narratives. Journal of Pediatric Oncology Nursing, 29, 151-160. doi:10.1177/1043454212446344

Enuku, C. A., \& Evawoma-Enuku, U. (2013). The need to incorporate reflective practice into nursing education in Nigeria. Journal of Nursing and Health Sciences, 1, 57-62. doi:10.9790/1959-0125762

Erikson, C., Salsberg, E., Forte, G., Bruinooge, S., \& Goldstein, M. (2007). Future supply and demand for oncologists: Challenges to assuring access to oncology services. Journal of Oncology Practice, 3, 79-86. doi:10.1200/JOP.0723601

Fejes, A. (2008). Governing nursing through reflection: A discourse analysis of reflective practices. Journal of Advanced Nursing, 64, 243-250. doi:10.1111/j.13652648.2008.04800.x

Forsey, M., Salmon, P., Eden, T., \& Young, B. (2013). Comparing doctors' and nurses' accounts of how they provide emotional care for parents of children with acute lymphoblastic leukemia. Psycho-Oncology, 22, 260-267. doi:10.1002/pon.2084

Fowler, J. (2014). Reflection: From staff nurse to nurse consultant. Part 5: Reflective practice. British Journal of Nursing, 23, 398. doi:10.12968/bjon.2014.23.7.398 
Franca, J. R. F. S., Costa, S. F. G., Lopez, M. E. L., Nobrega, M. M. L., \& Franca, I. S. X. (2013). The importance of communication in pediatric oncology palliative care: Focus on humanistic nursing theory. Revista Latino-America de Enfermagem, 21, 780-786. doi:10.1590/S0104-11692013000300018

Gallagher, R., \& Gormley, D. K. (2009). Perceptions of stress, burnout, and support systems in pediatric bone marrow transplantation nursing. Clinical Journal of Oncology Nursing, 13, 681-685. doi:10.1188/09.CJON.681-685

Geisler, P. R. (2015). Interprofessional education in athletic training education: The next wave (to tap the breaks on). International Journal of Athletic Therapy and Training, 20(6), 1-4. doi:10.1123/ijatt.2015-0084

Gelabert-Vilella, S., Bonmatí-Thomàs, A., Bosch-Farré, C., Malagón-Aguilera, C. M., FuentesPumarola, C., \& Ballester-Ferrando, D. (2014). Mental health nursing practicum: Student and mentor perspectives on stress and satisfaction. Journal of Technology and Science Education, 4, 79-88. doi:10.3926/jotse.96

Gibbs, G. (1988). Learning by doing: A guide to teaching and learning methods. Oxford, England: Oxford Polytechnic.

Graneheim, U., \& Lundman, B. (2004). Qualitative content analysis in nursing research: Concepts, procedures, and measures to achieve trustworthiness. Nurse Education Today, 24, 105-112. doi:10.1016/j.nedt.2003.10.001

Gustafsson, C., \& Fagerberg, I. (2004). Reflection, the way to professional development. Journal of Clinical Nursing, 13, 271-280. doi:10.1046/j.1365-2702.2003.00880.x

Halm, M. (2017). The role of mindfulness in enhancing self-care for nurses. American Journal of Critical Care, 26, 344-348. doi:10.4037/ajcc2017589 
Hartlage, H. N. (2012). Exploring boundaries in pediatric oncology nursing. Journal of Pediatric Oncology Nursing, 29, 109-112. doi:10.1177/1043454212438965

Hecktman, H. M. (2012). Stress in pediatric oncology nurses. Journal of Pediatric Oncology Nursing, 29, 356-361. doi:10.1177/1043454212458367

Heidegger, M. (1962). Being and time (J. Macquarrie \& E. Robinson, Trans.). Oxford, England: Blackwell.

Heller, B. R., Oros, M. T., \& Durney-Crowley, J. (2000). The future of nursing education: Ten trends to watch. Nursing and Health Care Perspectives, 21, 9-13. Retrieved from http://www.nln.org

Hentz, P. B., \& Lauterbach, S. S. (2005). Becoming self-reflective: Caring for self \& others. International Journal of Human Caring, 9, 24-28. Retrieved from https://www.humancaring.org/page-18066

Hinds, P. S., Quargnenti, A. G., Hickey, S. S., \& Mangum, G. H. (1994). A comparison of the stress-response sequence in new and experienced pediatric oncology nurses. Cancer Nursing, 17, 61-71. Retrieved from https://journals.lww.com/cancernursingonline

Hines, M. E., \& Gaughan, J. (2014). Pediatric nurses acknowledging praxis: Recognizing caring in reflective narratives. International Journal of Human Caring, 18(3), 26-35. doi:10.20467/1091-5710-18.3.26

Horton-Deutsch, S., \& Sherwood, S. (2008). Reflection: An educational strategy to develop emotionally competent nurse leaders. Journal of Nursing Management, 16, 946-954. doi:10.1111/j.1365-2834.2008.00957.x

Hsieh, H. F., \& Shannon, S. E. (2005). Three approaches to qualitative content analysis. Qualitative Health Research, 15, 1277-1288. doi:10.1177/1049732305276687 
Husserl, E. (1931/2012). Ideas: General introduction to pure phenomenology (Rev. ed.). New York City, NY: Routledge.

Jestico, E., \& Finlay, T. (2017). “A stressful and frightening experience?” Children's nurses' perceived readiness to care for children with cancer following pre-registration nurse education: A qualitative study. Nurse Education Today, 48, 62-66.

doi:10.1016/j.nedt.2016.09.019

Johns, C. (2009). Becoming a reflective practitioner. West Sussex, England: Blackwell.

Jonsson, A., \& Halabi, J. (2006). Work related post-traumatic stress as described by Jordanian emergency nurses. Accident and Emergency Nursing, 14(2), 89-96. doi:10.1016/j.aaen.2006.02.001

Joyce-McCoach, J. T., Parrish, D. R., Andersen, P. R., \& Wall, N. (2013). Unlocking reflective practice for nurses: Innovations in working with Master of Nursing students in Hong Kong. Nurse Education in Practice, 13, 388-392. doi:10.1016/j.nepr.2012.11.002

Karbasi, C., Pacheco, E., Bull, C., Evanson, A., \& Chaboyer, W. (2018). Registered nurses' provision of end-of-life care to hospitalised adults: A mixed studies review. Nurse Education Today, 71, 60-74. doi:10.1016/j.nedt.2018.09.007

Karimi, S., Haghani, F., Yamani, N., \& Kalyani, M. N. (2017). Exploring the perceptions of nursing students about consequences of reflection in clinical settings. Electronic Physician, 9, 5191-5198. doi:10.19082/5191

Kelsey, C., \& Hayes, S. (2015) Frameworks and models—-scaffolding or strait jackets? Problematising reflective practice. Nurse Education in Practice, 15, 393-396. doi:10.1016/j.nepr.2015.05.006 
Kerfoot, K. (1996). The emotional side of leadership: The nurse manager's challenge. Nursing Economics, 14, 59-61. Retrieved from http://www.nursingeconomics.net

King-Okoye, M., \& Arber, A. (2014). "It stays with me": The experiences of second- and thirdyear student nurses when caring for patients with cancer. European Journal of Cancer Care, 23, 441-449. doi:10.1111/ecc.12139

Kolb, D. (1984). Experiential learning: Experience in the source of learning and development. Englewood Cliffs, NJ: Prentice-Hall.

Koshy, K., Limb, C., Gundogan, B., Whitehurst, K., \& Jafree, D. J. (2017). Reflective practice in health care and how to reflect effectively. International Journal of Surgery: Oncology, 2(e20), 1-3. doi:10.1097/IJ9.0000000000000020

Krippendorf, K. (2004). Context analysis: An introduction to its methodology (2nd ed.). London, England: Sage.

Kushnir, T., Rabin, S., \& Azulai, S. (1997). A descriptive study of stress management in a group of pediatric oncology nurses. Cancer Nursing, 20, 414-421. doi:10.1097/00002820199712000-00005

Laposa, J. M., Alden, L. E., \& Fullerton, L. M. (2003). Work stress and posttraumatic stress disorder in ED nurses/personnel. Journal of Emergency Nursing, 29, 23-28. doi:10.1067/men.2003.7

Lazzarin, M., Biondi, A., \& Di Mauro, S. (2012). Moral distress in nurses in oncology and hematology. Nursing Ethics, 19, 183-195. doi:10.1177/0969733011416840

Lexico. (2019). Definition of intention in English. Retrieved from https://www.lexico.com/en/definition/intention 
Linder, L. (2009). Experiences of pediatric oncology nurses: The first year of hire. Journal of Pediatric Oncology Nursing, 26, 29-40. doi:10.1177/1043454208323913

Lopez, K. A., \& Willis, D. G. (2004). Descriptive versus interpretive phenomenology: Their contributions to nursing knowledge. Qualitative Health Research, 14, 726-735. doi: $10.1177 / 1049732304263638$

Macpherson, C. F. (2008). Peer-supported storytelling for grieving pediatric oncology nurses. Journal of Pediatric Oncology Nursing, 25, 148-163. doi:10.1177/1043454208317236

Mann, K., Gordon, J., \& MacLeod, A. (2009). Reflection and reflective practice in health professions education: A systematic review. Advances in Health Science Education, 14, 595-621. doi:10.1007/s10459-007-9090-2

Matua, G. A. (2015). Choosing phenomenology as a guiding philosophy for nursing research. Nursing Research, 22(4), 30-34. doi:10.7748/nr.22.4.30.e1325

Maxwell, J. A. (2005). Qualitative research design: An interactive approach (2nd ed.). Thousand Oaks, CA: Sage.

McGarth, D., \& Higgins, A. (2006). Implementing and evaluating reflective practice group sessions. Nurse Education in Practice, 6, 175-181. doi:10.1016/j.nepr.2005.10.003

Mirlashari, J., Warnock, F., \& Jahanbani, J. (2017). The experiences of undergraduate nursing students and self-reflective accounts of first clinical rotation in pediatric oncology. Nurse Education in Practice, 25, 22-28. doi:10.1016/j.nepr.2017.04.006

Moerer-Urdahl, T., \& Creswell, J. W. (2004). Using transcendental phenomenology to explore the "ripple effect" in a leadership mentoring program. The International Journal of Qualitative Methods, 3(2), 1-28. doi:10.1177/160940690400300202 
Montgomery, K. E., Sawin, K. J., \& Hendricks-Ferguson, V. (2017). Communication during palliative care and end of life: Perceptions of experienced pediatric oncology nurses. Cancer Nursing, 40(2), E47-E57. doi:10.1097/NCC.0000000000000363

Moody, K., Kramer, D., Santizo, R. O., Magro, L., Wyshogrod, D., Ambrosio, J., . . Stein, J. (2013). Helping the helpers: Mindfulness training for burnout in pediatric oncology —a pilot program. Journal of Pediatric Oncology Nursing, 30, 275-284. doi:10.1177/1043454213504497

Morrison, C. F., \& Morris, E. J. (2017). The practices and meanings of care for nurses working on a pediatric bone marrow transplant unit. Journal of Pediatric Oncology Nursing, 34, 214-221. doi:10.1177/1043454216688637

Morrison, S. M. (2011). An integrative review of expert nursing practice. Journal of Nursing Scholarship, 43, 163-170. doi:10.1111/j.1547-5069.2011.01398.x

Moustakas, C. (1994). Phenomenological research methods. Thousand Oaks, CA: Sage.

Mukherjee, S., Beresford, B., \& Tennant, A. (2014). Staff burnout in pediatric oncology: New tools to facilitate the development and evaluation of effective interventions. European Journal of Cancer Care, 23, 450-461. doi:10.1111/ecc.12176

Munhall, P. L. (2007). A phenomenological method. In P. L. Munhall (Ed.), Nursing research: A qualitative perspective (pp. 145-210). Sudbury, MA: Jones and Bartlett.

Oluwatoyin, F. E. (2015). Reflective practice: Implications for nurses. Journal for Nursing and Health Science, 4(4), 28-33. doi:10.9790/1959-04432833

O’Neill, L., Johnson, J., \& Mandela, R. (2019). Reflective practice groups: Are they useful for liaison psychiatry nurses working within the emergency department? Archives of Psychiatric Nursing, 33, 85-92. doi:10.1016/j.apnu.2018.11.003 
Papadatou, D. (2001). The grieving healthcare provider. Bereavement Care, 20(2), 26-29. doi:10.1080/02682620108657520

Papadatou, D., Bellali, T., Papazoglou, I., \& Petraki, D. (2002). Greek nurse and physician grief as a result of caring for children dying of cancer. Journal of Pediatric Nursing, 28, 345353. Retrieved from http://www.pediatricnursing.net

Pearson, H. N. (2013). "You've only got one chance do get it right”: Children's cancer nurses' experiences of providing palliative care in the acute hospital setting. Issues in Comprehensive Pediatric Nursing, 36, 188-211. doi:10.3109/01460862.2013.797520

Peden-McAlpine, C., Tomlinson, P. S., Forneris, S. G., Genck, G., \& Meiers, S. J. (2005). Evaluation of a reflective practice intervention to enhance family care. Journal of Advanced Nursing, 49, 494-501. doi:10.1111/j.1365-2648.2004.03322.x

Pool, N. M. (2018). Looking inward: Philosophical and methodological perspectives on phenomenological self-reflection. Nursing Science Quarterly, 31, 245-252. doi: $10.1177 / 0894318418774912$

Pritchard, M., \& Davies, B. (2002). End of life in pediatric oncology: How clinical practice leads to research. Journal of Pediatric Oncology Nurses, 19(6), 191-197. doi:10.1053/jpon.2002.127226

Pye, K. (2013). Exploring moral distress in pediatric oncology: A sample of registered practitioners. Issues in Comprehensive Pediatric Nursing, 36, 248-261. doi:10.3109/01460862.2013.812693

Rains, C. A., \& Robinson, B. (2010). School nurses and athletic trainers team up on concussion management. NASN School Nurse, 25, 234-238. doi:10.1177/1942602X10376672 
Rashotte, J., Fothergill-Bourbonnais, F., \& Chamberlain, M. (1997). Pediatric intensive care nurses and their grief experiences: A phenomenological study. Journal of Acute and Critical Care, 26, 372-386. doi:10.1016/S0147-9563(97)90024-8

Rees, K. L. (2013). The role of reflective practices in enabling final year nursing students to respond to the distressing emotional challenges of nursing work. Nurse Education in Practice, 13, 48-52. doi:10.1016/j.nepr.2012.07.003

Reid, F. C. (2013). Lived experiences of adult community nurses delivering palliative care to children and young people in rural areas. International Journal of Palliative Nursing, 19, 541-547. doi:10.12968/ijpn.2013.19.11.541

Rizzo, C. S., Brietbach, A. P., \& Richardson, R. (2015). Athletic trainers have a place in interprofessional education and practice. Journal of Interprofessional Care, 29, 256-257. doi:10.3109/13561820.2014.942778

Robert, R. R., \& Petersen, S. (2013). Critical thinking at the bedside: Providing safe passage to patients. MedSurg Nursing, 22, 85-93. Retrieved from https://www.amsn.org/professional-development/periodicals/medsurg-nursing-journal

Ruth-Sahd, L. A. (2003). Reflective practice: A critical analysis of data-based studies and implications or nursing education. Journal of Nursing Education, 42, 488-497. doi:10.3928/0148-4834-20031101-07

Sadler, G. R., Lee, H., Lim, R. S., \& Fullerton, J. (2010). Recruiting hard-to-reach United States population sub-groups via adaptations of snowball sampling strategies. Nursing \& Health Sciences, 12, 369-374. doi:10.1111/j.1442-2018.2010.00541.x.

Schön, D. A. (1983). The reflective practitioner: How professionals think in action. New York, NY: Basic Books. 
Schott Foundation. (2014). Restorative practices: Fostering healthy relationships \& promoting positive disciplines in schools. Retrieved from http://schottfoundation.org/sites/default/files/restorative-practices-guide.pdf

Shirley, M. R. (2006). Authentic leaders creating healthy work environments for nursing practice. American Journal of Critical Care, 15, 256-268. Retrieved from http://ajcc.aacnjournals.org/content/15/3/256.full.pdf + html

Smyth, J. (1989). Developing and sustaining critical reflection in teacher education. Journal of Teacher Education, 40(2), 2-9. doi:10.1177/002248718904000202

Solomon, J. L. (1987). From Hegel to existentialism. New York, NY: Oxford University.

Starks, H., \& Trinidad, S. B. (2007). Choose your method: A comparison of phenomenology, discourse analysis, and grounded theory. Qualitative Health Research, 17, 1372-1380. doi:10.1177/1049732307307031

Sultan, N. (2018). Heuristic inquiry: Researching human experience holistically. Thousand Oaks, CA: Sage.

Tanner, C. A. (2006). Thinking like a nurse: A research-based model of clinical judgment in nursing. Journal of Nursing Education, 45, 204-211. doi:10.3928/01484834-20060660104

van de Mortel, T. F. (2008). Faking it: Social desirability response bias in self-report research. Australian Journal of Advanced Nursing, 25(4), 40-48. Retrieved from http://www.ajan.com.au

van Manen, M. (1990). Researching lived experience: Human science for an action sensitive pedagogy. Albany: State University of New York Press. 
van Manen, M. (2014). Phenomenology of practice: Meaning-giving methods in phenomenological research and writing. New York, NY: Routledge.

van Stralen, S. (2002). Making sense of one's experience in the workplace. New Directions for Adult \& Continuing Education, 94, 13-21. doi:10.1002/ace.55

van Zyl, A. B., \& Noonan, I. (2018). The Trojan War inside nursing: An exploration of compassion, emotional labour, coping, and reflection. British Journal of Nursing, 27, 1192-1196. doi:10.12968/bjon.2018.27.20.1192

Vogenberg, F. R., \& Santilli, J. (2018). Healthcare trends for 2018. American Health \& Drug Benefits, 11, 48-54.

Walker, J. (2008). The nursing expert in clinical practice. Journal of Orthopaedic Nursing, 12, 512. doi:10.1016/j.joon.2007.08.016

Willis, D. G., Sullivan-Bolyai, S., Knalf, K., \& Cohen, M. Z. (2016). Distinguishing features and similarities between descriptive phenomenological and qualitative descriptive research. Western Journal of Nursing Research, 38, 1185-1204. doi:10.1177/0193945916645499

Wilson, K. (2005). The evolution of the role of nurses: The history of nurse practitioners in pediatric oncology. Journal of Pediatric Oncology Nursing, 22, 250-253. doi:10.1177/1043454205279288

Zadeh, S., Gamba, N., Hudson, C., \& Wiener, L. (2012). Taking care of care providers: A wellness program for pediatric nurses. Journal of Pediatric Oncology Nursing, 29, 294299. doi: $10.1177 / 1043454212451793$

Zander, M., Hutton, A., \& King, L. (2010). Coping and resilience factors in pediatric oncology nurses. Journal of Pediatric Oncology Nursing, 27, 94-108. doi: $10.1177 / 1043454209350154$ 
Zander, M., Hutton, A., \& King, L. (2013). Exploring resilience in pediatric oncology nursing staff. Collegian, 20, 17-25. doi:10.1016/j.colegn.2012.02.002 


\section{Appendices}

\section{Appendix A: Participant Packet}

\section{RECRUITMENT EMAIL to be distributed to potential participants}

Subject line: Seeking experienced pediatric oncology nurses, who self-identify with using selfreflective practices, to participate in a qualitative research study

Dear Potential Study Participant,

Hello, my name is Daniel Bonilla and I am a doctoral student in the Leadership Studies program at the College of Educational Studies at Chapman University. I am a health care educator and certified athletic trainer who is interested in the unique lived experiences of medical professionals who maintain employment and navigate the unique emotional landscape of pediatric oncology. You are receiving this email because you have been identified as an experienced pediatric oncology nurse currently employed at Loma Linda University Children's Hospital Pediatric Hematology Oncology Stem Cell Transplant Intensive Care Unit (Unit 4800) with at least 10 years of professional experience. Your email address was obtained from a current pediatric oncology nurse currently employed at Loma Linda University Children's Hospital. I would like to invite you to participate in a qualitative research study. This study is completely voluntary and your choice to participate will not affect the relationship with the researcher or Loma Linda University Children's Hospital.

As part of my dissertation, I am exploring how experienced pediatric oncology nurses (who selfidentify as being reflective) perceive and describe their lived experiences using self-reflective practices in patient care. We hope the findings from this investigation will provide insight as to how experienced pediatric oncology nurses develop self-reflective practices which provide them with the physical and emotional strength to last in their profession as specialty nurses even when faced with the death and dying of their patients.

If you agree to participate in this study, I will schedule an in-person interview with you at the time and location of your choice. Participating in the study will require your participation in three in-person interviews that will take approximately one or more hours. Throughout the study period you may be asked to participate in follow up interviews or answer follow up questions. To maintain confidentiality, you will not be identified by name on any form of recording; similarly, any identifying information that may arise during recording will be replaced in the final copies used for analysis. My dissertation committee and I will be the only persons to read the written data, though my professors will not be able to identify any of the participants by their actual names. When the written data have been completed, you may be asked to check to see if the transcript accurately represents what you have shared. At that time you will have the opportunity to add any additional comments that you like to the document or ask that topics be removed. All original copies of the recorded data will be kept locked, and within 10 years of when the study is finished all original audio files will be destroyed. Your name and any other information gathered in this study will remain confidential and will only be used for educational purposes. 
The information gathered from this investigation will be used for my dissertation, conference presentations, and publications that emerge from the findings of the dissertation. If, at a later date, I wish to use your data in new research, you will be contacted and given the same rights to refuse participation in the new study. If you have any questions, please contact me or my

Dissertation Chair: Dr. Whitney McIntyre Miller at 714-XXX-XXXX, xxxxx@chapman.edu. I appreciate your thoughtful consideration of my request. I look forward to your participation in the study.

With regards,

Daniel J. Bonilla

Daniel J. Bonilla, MS, ATC

Doctoral Student

Chapman University

College of Educational Studies

Email: xxxxx@mail.chapman.edu

Cell: 626-XXX-XXXX 


\section{CHAPMAN UNIVERSITY}

\section{CONSENT TO ACT AS A HUMAN RESAERCH SUBJECT}

Experienced Pediatric Oncology Nurses Using Self-Reflective Practices in the Clinical Setting: A Descriptive Phenomenological Investigation

You are being asked to participate in a research study. Participation is completely voluntary. Please read the information below and ask questions about anything that you do not understand. A researcher listed below will be available to answer your questions.

\section{RESEARCH TEAM}

Lead Researcher

Daniel J. Bonilla, Doctoral Student

College of Educational Studies

Cell: 626-XXX-XXXX and E-mail: xxxxx@mail.chapman.edu

Faculty Sponsor

Dr. Whitney McIntyre Miller

College of Educational Studies

Phone: 714-XXX-XXXX and E-mail: xxxxx@chapman.edu

STUDY LOCATION(S): TBD

STUDY SPONSOR(S): Chapman University

\section{Investigator Financial Conflict of Interest}

No one on the study team has a disclosable financial interest related to this research project.

\section{WHY IS THIS RESEARCH STUDY BEING DONE?}

The purpose of this research study is to explore how experienced pediatric oncology nurses describe their experiences using self-reflective practices in patient care. Due to the high demands of pediatric oncology nurses to provide patient care to a specific population an interview-based exploration of the lived experiences of participants is necessary to understand the unique selfreflective practices currently used among nurses. There is limited research and information on pediatric oncology nurses and how they choose particular reflective practices that help them cope. Understanding the lived experiences of pediatric oncology nurses and how they define, describe, and understand self-reflective practices may help future nursing educators illuminate specific characteristics and unique experiences that cultivate a lengthy career in pediatric oncology. The nursing profession, as well as the whole health care system, may benefit from understanding pediatric oncology nurses' experiences using self-reflective practices.

\section{HOW MANY PEOPLE WILL TAKE PART IN THIS STUDY?}

You will be one of five to 10 experienced pediatric oncology nurses who are currently employed at Loma Linda University Children's Hospital. Experienced will be defined as being employed 
as a pediatric oncology nurse for 5 or more years. Individual interviews will be conducted inperson at a location previously agreed upon by you and the researcher.

\title{
WHAT PROCEDURES ARE INVOLVED WITH THIS STUDY AND HOW LONG WILL THEY TAKE?
}

1. Daniel Bonilla will be the primary researcher. If you agree to participate, an individual interview time at your convenience will be scheduled with you via telephone or email. After the consent form has been signed and collected, you will be asked to complete a brief demographic questionnaire via email (e.g., age, years of experience, level of education). You will be asked to participate in three in-person interviews lasting approximately one or more hours, which will be audio recorded. The semi-structured interview will gather an understanding of your lived experiences with patients and the overall system of care as a pediatric oncology nurse. The interview protocol will be flexible (approximately 5-10 open-ended questions) to allow for the questions to evolve throughout the interview session. The questions will be provided in advance to stimulate self-reflection on each question prior to the scheduled interview.

2. Participation in the study will include a total of three interviews (one initial interview and two follow up interviews) and take a total of about 1 or more hours over a period of 3 to 4 months.

\begin{abstract}
AM I ELIGIBLE TO PARTICIPATE IN THIS STUDY?
Please note this may not be a complete list of eligibility criteria. We have included a few examples of study criteria to help you better understand how your eligibility in the study will be determined; your study team will go through the study eligibility criteria with you to verify if you qualify for participation in this study.
\end{abstract}

\section{Inclusion Requirements}

You can participate in this study if you are:

- Female or Male (18 years or older)

- A Registered Nurse and currently employed as a pediatric oncology nurse

- Have 5 or more years of experience as a pediatric oncology nurse

- Self-identify as being reflective in the process of patient care

\section{Exclusion Requirements}

You cannot participate in this study if you are:

- Not a Registered Nurse and or pediatric oncology nurse

- Have less than 5 years of pediatric oncology nursing experience

- Do not self-identify with being reflective in the process of patient care

\section{WHAT ARE THE POSSIBLE DISCOMFORTS OR RISKS RELATED TO THE STUDY?}

There are no known harms or discomforts associated with this study beyond those encountered in normal daily life. The possible risks and/or discomforts associated with the procedures described in this study include: loss of confidentiality and emotional discomfort when reflecting on your 
own lived experiences during the interview. If discomfort occurs, it should be brief and shortlived.

Breach of Privacy and Confidentiality: No personal information (i.e., your name) will be saved as a part of this research process. Audio files will be coded in a way that will protect your identity. These files will be stored on a personal laptop computer with password protection and the data discarded not later than 10 years after publication of this study. Your participation in the study will not be disclosed to any other individuals.

\section{ARE THERE BENEFITS TO TAKING PART IN THE STUDY? Participant Benefits}

The possible benefits you may experience from the procedures described in this study include increased awareness of your emotions regarding self-reflective practices, the capacity to selfreflect after experiencing strong emotions, and learning to pay attention to difficult situations and responding with social skills that enhance dignity and respect for others.

\section{Benefits to Others or Society}

Greater understanding of how experienced pediatric oncology nurses describe how they choose and use self-reflective practices in patient care.

\section{WILL I BE PAID FOR TAKING PART IN THIS STUDY?}

You will not be compensated for your participation in this research study.

\section{Reimbursement}

You will not be reimbursed for any out of pocket expenses, such as parking or transportation fees.

\section{WHAT HAPPENS IF I AM INJURED BECAUSE I TOOK PART IN THIS STUDY?} It is important that you promptly tell the researchers if you believe you have been injured because of taking part in this study. You can tell the researcher in person or call him/her at the number listed at the top of this form.

If you become ill or get injured as a result of this study you should seek medical treatment through your doctor or treatment center or choice. The University and/or researchers will not pay for medical care required because of a bad outcome resulting from your participation in this research study. This does not keep you from seeking to be paid back for care required because of a bad outcome.

\section{WHAT HAPPENS IF I WANT TO STOP TAKING PART IN THIS STUDY?}

You are free to withdraw from this study at any time. If you decide to withdraw from this study you should notify the research team immediately. The research team may also end your participation in this study if you do not follow instructions, miss scheduled visits, or if your safety and welfare are at risk. 


\section{HOW WILL MY PERSONAL INFORMATION BE KEPT?}

All information will be kept confidential and will be reported in an anonymous fashion, and that the recordings will be erased 10 years after the study has been completed.

\section{WHO WILL HAVE ACCESS TO MY STUDY DATA?}

The research team, authorized Chapman University personnel, and regulatory entities such as the Office of Human Research Protections (OHRP), may have access to your study records to protect your safety and welfare.

Any information derived from this research project that personally identifies you will not be voluntarily released or disclosed by these entities without your separate consent, except as specifically required by law. Study records provided to authorized, non-Chapman University entities will not contain identifiable information about you; nor will any publications and/or presentations without your separate consent.

While the research team will make every effort to keep your personal information confidential, it is possible that an unauthorized person might see it. We cannot guarantee total privacy.

\section{WHO CAN ANSWER MY QUESTIONS ABOUT THE STUDY?}

If you have questions, concerns, or complaints, or think the research has hurt you, talk to the research team at Daniel Bonilla 626-XXX-XXXX or Dr. Whitney McIntyre Miller $714-X X X$ $X X X X$.

This research has been reviewed and approved by an Institutional Review Board ("IRB"). You may talk to them at 714-628-2833 or mailto:irb@chapman.edu if:

- Your questions, concerns, or complaints are not being answered by the research team.

- You cannot reach the research team.

- You want to talk to someone besides the research team.

- You have questions about your rights as a research participant.

- You want to get information or provide input about this research.

\section{HOW DO I AGREE TO PARTICIPATE IN THIS STUDY?}

You should not sign this consent form until all of your questions about this study have been answered by a member of the research team listed at the top of this form. You will be given a copy of this signed and dated consent form to keep.

Participation in this study is voluntary. You may refuse to answer any question or discontinue your involvement at any time without penalty or loss of benefits to which you might otherwise be entitled. Your decision will not affect your future relationship with Chapman University. 


\section{AUDIO RECORDING:}

I have received an adequate description of the purpose and procedures for audio-recording sessions during the course of the proposed research. I give my consent to allow myself to be audio-recorded during participation in this study, and for those records to be reviewed by persons involved in the study, as well as for other professional purposes as described to me.

Yes, I agree to allow the research team to audio record my interview(s).

No, I do not wish to have my interview audio recorded.

Signature of Participant

Date

Your signature below indicates you have read the information in this consent form and have had a chance to ask any questions you have about this study.

\section{I agree to participate in the study.}

Subject Signature

Date

Printed Name of Subject

Researcher Signature

Date

Printed Name of Researcher 


\section{CHAPMAN UNIVERSITY}

\section{Research Participant's Bill of Rights}

\section{The rights listed below are the right of every individual asked to participate in a research}

study. You have the right:

1. To be told about the nature and purpose of the study.

2. To be told about the procedures to be followed in the research study, and whether any of the drugs, devices, or procedures is different from what would be used in standard practice.

3. To receive a description of any side effects, discomforts, or risks that you can reasonably expect to occur during the study.

4. To be told of any benefits that you may reasonably expect from the participation in the study, if applicable.

5. To receive a description of any alternative procedures, drugs, or devices that might be helpful, and their risks and benefits compared to the proposed procedures, drugs or devices.

6. To be told of what sort of medical treatment, if any, will be available if any complications should arise.

7. To be given a chance to ask any questions concerning the research study both before agreeing to participate and at any time during the course of the study.

8. To refuse to participate in the research study. Participation is voluntary. You may refuse to answer any question or discontinue your involvement at any time without penalty or loss of benefits to which you might otherwise be entitled. Your decision will not affect your right to receive the care you would receive if you were not in the experiment.

9. To receive a copy of the signed and dated written consent form and a copy of this form.

10. To be given the opportunity to freely decide whether or not to consent to the research study without any force, coercion, or undue influence.

If you have any concerns or questions regarding the research study you should contact the research team listed at the top of the consent form.

If you are unable to reach a member of the research team and have general questions, or you have concerns or complaints about the research study, research team, or questions about your rights as a research subject, please contact the Chapman University IRB staff at 714-628-2833 or irb@chapman.edu. 


\section{Appendix B: Interview Protocol}

Equipment: audio-recording device, interview guide, journal/notes, etc.

*Length of each interview: will vary so make sure to schedule each co-researcher with enough time to adequately answer each question to the best of their abilities.

\section{Research Question and Interview Guide}

Research question: what is the lived experience of EPONs using SRP in patient care? Interview 1 (up to 1 hour or more) Primary Researcher to Participants:

*Begin interview with informal conversation and ask participants about their day/weekend. "Welcome and thank you for consenting to participant in this study. As part of my dissertation, I am exploring how experienced pediatric oncology nurses (EPONs) - who self-identify as being reflective - perceive and describe their lived experiences using self-reflective practices in patient care. Self-reflective practices are unique and occur when an individual consciously explores an experience by questioning, for example, what happened, what was my role in this experience, what behaviors, thinking patterns, and emotions am I experiencing (Oluwatoyin, 2015)? Currently, there is limited information regarding the unique experiences of EPONs using selfreflective practices in the clinical setting. The purpose of this research study is to explore how you describe your experience(s) and the meaning of using self-reflective practices in patient care. I will be using a digital audio-recording device and will record our interview. Although you already signed the consent form, I would still like to get your verbal consent regarding audiorecording our interview. Do you consent to audio-recording? (Pause for participant's responses) . .. If yes, I will continue the interview by asking questions and seeking vivid, accurate, portrayals of what these experiences were like for you: your thoughts, feelings, and behaviors, as well as situations, events, places, and people connected with your experience. Remember that participation is completely voluntary. If you wish to stop or end your participation in this study, you are free to do so at any time. If you decide to stop the interview for personal reasons and would like to reschedule your interview for a later time and date, you may do so at any time. There will be three total interviews and each interview will require at least an hour of your time. If you have any questions or concerns prior to commencing the interview, please feel free to ask me now. If no comments, questions or concerns, we will begin the first interview." Please take a few moments to reflect-on your particular experiences and tell me ...

"Contexualization" and eliciting the "lifeworld" of the participant using descriptive/narrative context questions (Bevan, 2014).

1 . . . about how you came to be employed or interested to work at this particular hospital/unit.

2. Tell me your experience of a typical day in the hospital/unit.

3. If you could define SRP in the clinical setting from your perspective, what would that be and why? Please reflect-on past experiences and tell me about how you developed an understanding of using self-reflective practices.

4. What is reflective practice an experience of? Please describe how you have used self-reflective practices in patient care?

-What does this mean to you?

5. How does using self-reflective practice in patient care impact your well-being?

-Please reflect-on and describe an experience(s) of that particular impact. 
6. As an PON, how do you use self-reflective practices during and after your shift?

-What does this mean to you?

"Apprehending the phenomenon or modes of appearing in the natural attitude using descriptive and structural questions" (Bevan, 2014).

7. As an PON, tell me about a patient care experience(s) and describe the type(s) of individual self-reflective practices you used to manage stress.

8. As an PON, tell me about the advantages or benefits of managing stress by using selfreflective practices in patient care ... tell me about the disadvantages or obstacles of managing stress by using self-reflective practices in patient care.

"Clarifying the phenomenon and developing meaning by using imaginative variation (varying the structure of questions)" (Bevan, 2014).

9. Reflect-on your experience(s) and describe how using self-reflective practices in patient care would change if your unit required all nurses to take time for their own physical and emotional wellbeing.

10. Describe how staff can use self-reflective practices in patient care to manage grief, moral distress, and burnout.

11. Do you believe self-reflective practices would play an important role for novice nurses? If you do believe it [self-reflective] plays an important role for novice nurses, in what ways? "Thank you for participating in this interview. Our first interview is complete. I will begin transcribing this interview immediately into a Word document. Upon completing the transcription, you will receive the complete interview transcription via your personal email. Please take time to review the entire transcription prior to our next meeting to make sure the interview was transcribed accurately and in the same words you can remember using. If you have any comments, concerns, or questions regarding the transcription of the first interview you may add comments by highlighting the specific section and adding a comment next to the area of concern. Please "Bold" your comments or questions to identify the addition of your comment. NOTE: You will receive a detailed transcription via email after each interview for your own records (three total). All three transcriptions will be used as data and will be analyzed and interpreted by me, the primary researcher, to develop a rich description of the live experience(s) of EPONs using self-reflective practices in patient care. At this time, we can schedule our second interview or we can schedule the next interview at your earliest convenience. The second interview will be used to clarify any comments or specific experiences you mentioned in the first interview. The second interview may last at least an hour long. This completes our first interview, thank you for your time. See you soon!"

\footnotetext{
*Interview 2 (up to 1 hour or more)

Primary Researcher to Co-Researchers:

*Begin interview with informal conversation and ask co-researchers about their day/weekend.

"Welcome and thank you for consenting to participant in this study. I will be using a digital audio-recording device and will record our interview. Although you already signed the consent form, I would still like to get your consent regarding audio-recording our interview. Do you consent to audio-recording? (Pause for participant's response) ... If yes, I will continue the second interview by asking questions and seeking vivid, accurate, portrayals of what these experiences were like for you: your thoughts, feelings, and behaviors, as well as situations, events, places, and people connected with your experience. Remember that participation is completely voluntary. If you wish to stop or end your participation in this study, you are free to do so at any time. If you decide to stop the second interview for personal reasons and would like
} 
to reschedule your interview for a later time and date, you may do so at any time. This is the second interview of three."

*This second interview will be used to gather more information regarding those particular experiences participant's previously mentioned. In this section, participants will be asked follow up questions that pertain to questions 7-11: "As you may remember the first interview, we discussed [in this section I will use information specific to each participant] . . ."

7. What additional details can you recall regarding the particular patient care experience(s) you previously described and type(s) of individual self-reflective practices you use to manage stress. 8. What additional details can you recall regarding the advantages/benefits or disadvantages/obstacles of managing stress by using self-reflective practices in patient care. 9. Reflect-on your experience(s) and describe additional details you may have regarding how using self-reflective practices in patient care would change if your unit required all nurses to take time for their own physical and emotional wellbeing.

10. Describe additional details you may have regarding how staff can use self-reflective practices in patient care to manage grief, moral distress, and burnout.

11. According to your last response, you believe self-reflective practices would play an important role for novice nurses? At this time, please feel free to add any details to support this claim.

\section{END OF INTERVIEW 2}

"Thank you for participating in this interview. Our second interview is complete. I will begin transcribing this interview immediately into a Word document. Upon completing the transcription, I will use these data to add or expand on the experiences you previously mentioned during the first interview. Once the second transcript has been completed, you will receive the second interview transcription via your personal email. Please take time to review the entire transcription prior to our final meeting to make sure the interview was transcribed accurately and in the same words you can remember using. If you have any comments, concerns, or questions regarding the transcription of the first or second interview you may add comments by highlighting the specific section and adding a comment next to the area of concern. Please "Bold" your comments or questions to identify the addition of your comment. At this time, we can schedule our third and final interview or we can schedule the interview at your earliest convenience. The third interview will be used to clarify any comments or specific experiences you mentioned in the first and or second interviews. The third interview may last less than an hour. This completes our second interview, thank you for your time. See you soon!"

\section{*Interview 3 (1 hour or less)- Member checking Primary Researcher to Co-Researchers:}

*Begin interview with informal conversation and ask co-researchers about their day/weekend. "Welcome and thank you for consenting to participant in this study. I will be using a digital audio-recording device and will record our interview. Although you already signed the consent form, I would still like to get your consent regarding audio-recording our interview. Do you consent to audio-recording? (Pause for participant's response) ... If yes, I will continue the third and final interview by asking questions and seeking vivid, accurate, portrayals of what these experiences were like for you: your thoughts, feelings, and behaviors, as well as situations, events, places, and people connected with your experience. Remember that participation is completely voluntary. If you wish to stop or end your participation in this study, you are free to do so at any time. If you decide to stop the final interview for personal reasons and would like to 
reschedule your interview for a later time and date, you may do so at any time. This is the final interview. If you can remember the first and second interviews, we discussed (information specific to each participant) ... "“

*This third interview will be considered a "member checking round" in which the previous information participant's mentioned during interviews one and two can be expanded upon or clarified at this time.

"... After reviewing the transcripts pertaining to your first and second interviews, if you wish to add information regarding your particular experience(s) using self-reflective practices in patient care you are free to do so at this time."

*If participants do not wish to add any more information, or feel that the first and second interviews were authentic representations of their lived experiences than the third interview will come to an end.

"Thank you for participating in this third and final interview. You have completed all three interviews. If you used this third interview to add to the experiences you mentioned during the first and second interviews, I will transcribe the information of this interview immediately into a Word document and will add it to the information collected in the previous interviews. Once the final transcript has been completed, you will receive the final interview transcription via your personal email, which will include all the information gathered from interviews one, two and three. Please take time to review the entire transcript to make sure all three interviews were transcribed accurately and in the same words you can remember using. If you have any comments, concerns, or questions regarding the final transcription you may add comments by highlighting the specific section and adding a comment next to the area of concern. Please "Bold" your comments or questions to identify the addition of your comment. This completes our final interview, thank you for your time. 